\title{
Preparation of Heterobimetallic Ketimido-Actinide-Molybdenum Complexes
}

Alexander J. Ayres, ${ }^{\mathrm{a}}$ Ashley J. Wooles, ${ }^{\mathrm{b}}$ Markus Zegke, ${ }^{\mathrm{b}}$ Floriana Tuna, ${ }^{\mathrm{c}}$ and Stephen T. Liddle ${ }^{\mathrm{b}}$

a School of Chemistry, The University of Nottingham, University Park, Nottingham, NG7 2RD, UK.

b Department of Chemistry, The University of Manchester, Oxford Road, Manchester, M13 9PL, UK.

c Department of Chemistry and Photon Science Institute, The University of Manchester, Oxford Road, Manchester, M13 9PL, UK.

*E-mail: steve.liddle@manchester.ac.uk

\section{Synthetic Methods}

\section{Preparation of DippN(H)PiPr 2}

${ }^{n} \mathrm{BuLi}$ (4.4 mL, 2.5 M in hexanes, $11 \mathrm{mmol}$ ) was added to a solution of 2,6-diisopropylaniline (1.77 g, $10.0 \mathrm{mmol})$ in hexanes $(30 \mathrm{~mL})$. The resulting solution was allowed to warm slowly to room temperature then stirred for 16 hours. The white precipitate was isolated via filtration and slurried in $\mathrm{Et}_{2} \mathrm{O}(30 \mathrm{~mL})$. The resulting slurry was added to a stirring solution of $\mathrm{P}^{i} \mathrm{Pr}_{2} \mathrm{Cl}(1.52 \mathrm{~g}, 10 \mathrm{mmol})$ in $\mathrm{Et}_{2} \mathrm{O}(30 \mathrm{ml})$ at $-78^{\circ} \mathrm{C}$. The solution was allowed to warm slowly to room temperature and stirred for a further hour to afford a pale yellow solution and a white precipitate. The reaction mixture was filtered away from the $\mathrm{LiCl}$ and the volatiles were removed in vacuo to give $\operatorname{DippN}(\mathrm{H}) \mathrm{P}^{\mathrm{i}} \mathrm{Pr}_{2}$ as a pale yellow oil. Yield: $2.61 \mathrm{~g}, 89 \% .{ }^{1} \mathrm{H}$ NMR $\left(\mathrm{C}_{6} \mathrm{D}_{6}, 295 \mathrm{~K}\right): \delta(\mathrm{ppm}) 1.01\left(12 \mathrm{H}, \mathrm{m},{ }^{i} \mathrm{Pr}-\mathrm{CH}_{3}\right), 1.29$ 
$\left(12 \mathrm{H}, \mathrm{d},{ }^{3} J_{\mathrm{HH}}=8.0 \mathrm{~Hz},{ }^{i} \mathrm{Pr}-\mathrm{CH}_{3}\right), 1.61\left(2 \mathrm{H}, \mathrm{m},{ }^{i} \mathrm{Pr}-\mathrm{CH}\right), 3.54\left(1 \mathrm{H}, \mathrm{d},{ }^{2} J_{\mathrm{PH}}=8.0 \mathrm{~Hz}, \mathrm{NH}\right), 3.66(2 \mathrm{H}$, $\left.\mathrm{m},{ }^{i} \operatorname{Pr}-\mathrm{CH}\right), 7.01-7.14(3 \mathrm{H}, \mathrm{m}, \mathrm{Ar}-\mathrm{C} H) .{ }^{13} \mathrm{C}\left\{{ }^{1} \mathrm{H}\right\} \mathrm{NMR}\left(\mathrm{C}_{6} \mathrm{D}_{6}, 295 \mathrm{~K}\right): \delta(\mathrm{ppm}) 17.17\left(\mathrm{~d}, J_{\mathrm{PC}}=10.0\right.$ $\left.\mathrm{Hz},{ }^{i} \operatorname{Pr}-\mathrm{CH}_{3}\right), 18.62\left(\mathrm{~d},{ }^{2} J_{\mathrm{PC}}=16.0 \mathrm{~Hz},{ }^{i} \operatorname{Pr}-\mathrm{CH}_{3}\right), 23.82\left({ }^{i} \operatorname{Pr}-\mathrm{CH}\right), 28.10\left({ }^{i} \mathrm{Pr}-\mathrm{CH}\right), 122.45(\mathrm{Ar}-\mathrm{CH})$, $123.90(\mathrm{Ar}-\mathrm{CH}), 139.58(\mathrm{Ar}-\mathrm{C}), 141.3\left(\mathrm{~d},{ }^{2} J_{P C}=7.0 \mathrm{~Hz}, \operatorname{Ar}-C\right) .{ }^{31} \mathrm{P}\left\{{ }^{1} \mathrm{H}\right\} \mathrm{NMR}\left(\mathrm{C}_{6} \mathrm{D}_{6}, 295 \mathrm{~K}\right): \delta$ (ppm) $67.14(\mathrm{~s})$.

\section{Preparation of TrippN(H)PiPr}

${ }^{n} \mathrm{BuLi}(4.4 \mathrm{~mL}, 2.5 \mathrm{M}$ in hexanes, $11 \mathrm{mmol})$ was added to a cold $\left(0^{\circ} \mathrm{C}\right)$ solution of 2,4,6triisopropylaniline $(2.19 \mathrm{~g}, 10.0 \mathrm{mmol})$ in hexanes $(30 \mathrm{~mL})$. The resulting solution was allowed to warm slowly to room temperature then stirred for 16 hours. The white precipitate was isolated via filtration and slurried in $\mathrm{Et}_{2} \mathrm{O}(30 \mathrm{ml})$. The resulting slurry was added to a stirring solution of ${ }^{i} \operatorname{Pr}_{2} \mathrm{Cl}(1.52 \mathrm{~g}, 10 \mathrm{mmol})$ in $\mathrm{Et}_{2} \mathrm{O}\left(30 \mathrm{~mL}\right.$ at $-78^{\circ} \mathrm{C}$. The solution was allowed to warm slowly to room temperature and stirred for a further hour to afford a pale yellow solution and a white precipitate. The reaction mixture was filtered away from the $\mathrm{LiCl}$ and the volatiles were removed in vacuo to give TrippN(H)PiPr 2 as a pale yellow oil. Yield: $3.11 \mathrm{~g}, 92 \% .{ }^{1} \mathrm{H}$ NMR $\left(\mathrm{C}_{6} \mathrm{D}_{6}, 295 \mathrm{~K}\right)$ : $\delta$ (ppm) 1.00-1.06 (12H, m, $\left.{ }^{i} \mathrm{Pr}-\mathrm{CH}_{3}\right), 1.26\left(6 \mathrm{H}, \mathrm{d},{ }^{3} \mathrm{~J}_{\mathrm{HH}}=6.0 \mathrm{~Hz},{ }^{i} \mathrm{Pr}-\mathrm{CH}_{3}\right), 1.34\left(12 \mathrm{H}, \mathrm{d},{ }^{3} J_{\mathrm{HH}}=9.0\right.$ $\left.\mathrm{Hz},{ }^{i} \operatorname{Pr}-\mathrm{CH}_{3}\right), 1.62\left(2 \mathrm{H}, \mathrm{m},{ }^{i} \mathrm{Pr}-\mathrm{CH}\right), 2.83\left(1 \mathrm{H}, \mathrm{m},{ }^{i} \operatorname{Pr}-\mathrm{CH}\right), 3.44\left(1 \mathrm{H}, \mathrm{d},{ }^{2} J_{P H}=6.0 \mathrm{~Hz}, \mathrm{NH}\right), 3.69$ $\left(2 \mathrm{H}, \mathrm{m},{ }^{i} \mathrm{Pr}-\mathrm{CH}\right) 7.11(2 \mathrm{H}, \mathrm{s}, \mathrm{Ar}-\mathrm{CH}) .{ }^{13} \mathrm{C}\left\{{ }^{1} \mathrm{H}\right\} \mathrm{NMR}\left(\mathrm{C}_{6} \mathrm{D}_{6}, 295 \mathrm{~K}\right): \delta(\mathrm{ppm}) 17.30\left(\mathrm{~d},{ }^{2} J_{\mathrm{PC}}=42.0\right.$ $\left.\mathrm{Hz}, \mathrm{P}-{ }^{i} \operatorname{Pr}-\mathrm{CH}_{3}\right), 18.61\left(\mathrm{~d}, J_{\mathrm{PC}}=66.0 \mathrm{~Hz}, \mathrm{P}-{ }^{i} \mathrm{Pr}-\mathrm{CH}\right) 24.14\left(\mathrm{~m}-{ }^{\mathrm{i}} \mathrm{Pr}-\mathrm{CH}_{3}\right), 27.94\left({ }^{i} \mathrm{Pr}-\mathrm{CH}_{3}\right), 28.18\left({ }^{i} \operatorname{Pr}-\right.$ $\mathrm{CH}), 28.24\left({ }^{i} \mathrm{Pr}-\mathrm{CH}\right), 34.17\left({ }^{i} \mathrm{Pr}-\mathrm{CH}\right), 121.61(\mathrm{Ar}-\mathrm{CH}), 139.04(\mathrm{Ar}-\mathrm{C}), 139.70(\mathrm{Ar}-\mathrm{C}), 142.19$ (ArC). ${ }^{31} \mathrm{P}\left\{{ }^{1} \mathrm{H}\right\} \operatorname{NMR}\left(\mathrm{C}_{6} \mathrm{D}_{6}, 295 \mathrm{~K}\right): \delta(\mathrm{ppm}) 66.37$ (s).

\section{Preparation of TrippN(H)PPh}

$\mathrm{PPh}_{2} \mathrm{Cl}(4.41 \mathrm{~g} 20 \mathrm{mmol})$ was added to a cold $\left(0^{\circ} \mathrm{C}\right)$ stirring solution of 2,4,6-triisopropylaniline (4.39 g, $20 \mathrm{mmol})$ and triethylamine $(2.02 \mathrm{~g} 20 \mathrm{mmol})$ in DCM. The mixture was allowed to warm slowly to room temperature and stirred for a further 3 hours and then volatiles were then removed in 
vacuo. The pale yellow solid was extracted into $\mathrm{Et}_{2} \mathrm{O}$ and filtered. Volatiles were removed in vacuo to afford TrippN(H)PPh 2 as a pale yellow solid. Yield: $7.82 \mathrm{~g}, 97 \% .{ }^{1} \mathrm{H} \mathrm{NMR}\left(\mathrm{C}_{6} \mathrm{D}_{6}, 295 \mathrm{~K}\right): \delta$ (ppm) $1.17\left(12 \mathrm{H}, \mathrm{d},{ }^{3} J_{\mathrm{HH}}=8.0 \mathrm{~Hz}\right.$, Tripp- $\left.\mathrm{CH}_{3}\right), 1.24\left(6 \mathrm{H}, \mathrm{d},{ }^{3} J_{\mathrm{HH}}=8.0 \mathrm{~Hz}\right.$, Tripp-CH $), 2.81(1 \mathrm{H}$, sept, ${ }^{3} J_{\mathrm{HH}}=8.0 \mathrm{~Hz}$, Tripp-CH), $3.24\left(2 \mathrm{H}, \mathrm{m}\right.$, Tripp-CH), $3.83\left(1 \mathrm{H}, \mathrm{d},{ }^{2} J_{\mathrm{PH}}=12.0 \mathrm{~Hz}, \mathrm{NH}\right), 7.09(2 \mathrm{H}$, m, Ar-CH), $7.11(4 \mathrm{H}, \mathrm{m}, \mathrm{Ar}-\mathrm{CH}), 7.12(2 \mathrm{H}, \mathrm{m}, \mathrm{Ar}-\mathrm{CH}), 7.50(4 \mathrm{H}, \mathrm{m}, \mathrm{Ar}-\mathrm{CH}) .{ }^{31} \mathrm{P}\left\{{ }^{1} \mathrm{H}\right\} \mathrm{NMR}$ $\left(\mathrm{C}_{6} \mathrm{D}_{6}, 295 \mathrm{~K}\right): \delta(\mathrm{ppm}) 42.10(\mathrm{~s})$.

\section{Preparation of $\left.\left[\mathrm{K}(\mathrm{Mes} N \mathrm{PPh})_{2}\right)\left(\mathrm{C}_{6} \mathrm{H}_{5} \mathrm{CH}_{3}\right)\right](\mathrm{S} 1)$}

To a stirring mixture of $\mathrm{MesN}(\mathrm{H}) \mathrm{PPh}_{2}(3.20 \mathrm{~g}, 10 \mathrm{mmol})$ and $\mathrm{KH}(0.40 \mathrm{~g}, 10 \mathrm{mmol})$ at $-78^{\circ} \mathrm{C}$ was added $\mathrm{Et}_{2} \mathrm{O}(40 \mathrm{~mL})$. The reaction mixture was allowed to warm slowly to room temperature and stirred for 12 hours. Volatiles were removed in vacuo and the resulting colourless solid was extracted into toluene to afford $\left[\mathrm{K}\left(\mathrm{MesNPPh}_{2}\right)\right]$ as a colourless crystalline solid. Colourless crystals of S1 suitable for a single crystal X-ray diffraction study were obtained from storage of a concentrated toluene solution at $-30{ }^{\circ} \mathrm{C}$ for 72 hours. Yield: $2.71 \mathrm{~g}, 76 \%$. Anal. Calc'd for $\mathrm{C}_{21} \mathrm{H}_{21} \mathrm{KNP}$ C, 70.56; H, 5.92; N, $3.92 \%$. Found: C, 70.43; H, 5.82; N, $3.91 \% .{ }^{1} \mathrm{H}$ NMR $\left(\mathrm{D}_{8}-\mathrm{THF}\right.$, $295 \mathrm{~K}): \delta(\mathrm{ppm}) 2.08\left(3 \mathrm{H}, \mathrm{s}, \mathrm{Mes}-\mathrm{CH}_{3}\right), 2.20\left(6 \mathrm{H}, \mathrm{s}, \mathrm{Mes}-\mathrm{CH}_{3}\right), 6.56(2 \mathrm{H}, \mathrm{s}, \mathrm{Ar}-\mathrm{CH}), 7.02(2 \mathrm{H}, \mathrm{m}$, Ar-CH), $7.14(4 \mathrm{H}, \mathrm{m}, \mathrm{Ar}-\mathrm{CH}), 7.62\left(4 \mathrm{H}, \mathrm{t},{ }^{2} \mathrm{~J}_{H}=4.0 \mathrm{~Hz}, \operatorname{Ar}-\mathrm{CH}\right) .{ }^{13} \mathrm{C}\left\{{ }^{1} \mathrm{H}\right\} \mathrm{NMR}\left(\mathrm{D}_{8}-\mathrm{THF}, 295 \mathrm{~K}\right)$ :

$\delta(\mathrm{ppm}) 19.90\left(\mathrm{Mes}-\mathrm{CH}_{3}\right), 20.54\left(\mathrm{Mes}-\mathrm{CH}_{3}\right), 125.07(\mathrm{Ar}-\mathrm{CH}), 126.74(\mathrm{~d}, \mathrm{JPC}=5.0 \mathrm{~Hz}(\mathrm{Ar}-\mathrm{CH})$, $127.94(\mathrm{Ar}-\mathrm{CH}), 128.30(\mathrm{Ar}-\mathrm{CH}), 128.71(\mathrm{Ar}-C) 130.41(\mathrm{Ar}-C), 130.6(\mathrm{Ar}-\mathrm{CH}), 137.47(\mathrm{Ar}-\mathrm{C})$. ${ }^{31} \mathrm{P}\left\{{ }^{1} \mathrm{H}\right\}$ NMR (D $\left.-\mathrm{THF}, 295 \mathrm{~K}\right): \delta(\mathrm{ppm}) 45.36$ (s). FTIR (Nujol): $\tilde{\mathrm{v}}\left(\mathrm{cm}^{-1}\right) .701$ (w), 739 (w), 767 (m), $862(\mathrm{~m}), 902(\mathrm{w}), 961(\mathrm{~m}), 1082(\mathrm{~m}), 1150(\mathrm{~m}), 1239(\mathrm{w}), 1295(\mathrm{~m}), 1413(\mathrm{~m})$.

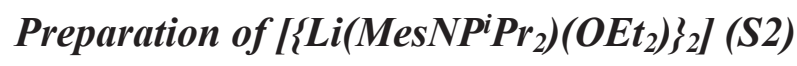

${ }^{n} \mathrm{BuLi}(13.2 \mathrm{ml}, 2.5 \mathrm{M}$ in hexanes, $33 \mathrm{mmol})$ was added to a pre-cooled $\left(-78{ }^{\circ} \mathrm{C}\right)$ solution of $\operatorname{MesN}(\mathrm{H}) \mathrm{P}^{i} \operatorname{Pr}_{2}(7.53 \mathrm{~g}, 30.0 \mathrm{mmol})$ in $\mathrm{Et}_{2} \mathrm{O}(75 \mathrm{ml})$. The resulting yellow solution was allowed to warm slowly to room temperature and stirred for 3 hours. The reaction mixture was concentrated in 
vacuo and cooled to $-30{ }^{\circ} \mathrm{C}$ to afford $\left[\mathrm{Li}\left(\mathrm{MesNP}^{\mathrm{i}} \mathrm{Pr}_{2}\right)\right]$ as a colourless solid. Colourless crystals of S2 suitable for a single crystal X-ray diffraction study were obtained from storage of a concentrated $\mathrm{Et}_{2} \mathrm{O}$ solution at $-30{ }^{\circ} \mathrm{C}$ for 72 hours. Yield: $7.75 \mathrm{~g}, 78 \%$. Anal. Calc'd for $\mathrm{C}_{15} \mathrm{H}_{25} \mathrm{LiNP}$ : C, 70.03; H, 9.79; N, 5.44 \%. Found: C, 70.08; H, 9.91; N, $5.58 \%$ \%. ${ }^{1} \mathrm{H}$ NMR (D $\left.-\mathrm{THF}, 295 \mathrm{~K}\right): \delta$ (ppm) 0.91 (6H, m, $\left.{ }^{i} \mathrm{Pr}-\mathrm{CH}_{3}\right), 1.10\left(6 \mathrm{H}, \mathrm{m},{ }^{i} \mathrm{Pr}-\mathrm{CH}_{3}\right) 1.42\left(2 \mathrm{H}, \mathrm{m},{ }^{i} \mathrm{Pr}-\mathrm{CH}\right) 2.06\left(3 \mathrm{H}, \mathrm{s}, \mathrm{Mes}-\mathrm{CH}_{3}\right), 2.32(6 \mathrm{H}, \mathrm{s}$, Mes- $\left.\mathrm{CH}_{3}\right), 6.52(2 \mathrm{H}, \mathrm{s}, \mathrm{Ar}-\mathrm{CH}) .{ }^{13} \mathrm{C}\left\{{ }^{1} \mathrm{H}\right\} \mathrm{NMR}\left(\mathrm{D}_{8}-\mathrm{THF}, 295 \mathrm{~K}\right): \delta(\mathrm{ppm}) 16.21\left(\mathrm{~d},{ }^{2} J_{\mathrm{PC}}=11.0 \mathrm{~Hz}\right.$, $\left.{ }^{i} \operatorname{Pr}-\mathrm{CH}_{3}\right), 17.33\left(\mathrm{~d},{ }^{2} \mathrm{~J}_{\mathrm{PC}}=19.0 \mathrm{~Hz},{ }^{i} \mathrm{Pr}-\mathrm{CH}_{3}\right), 17.95\left(\mathrm{Mes}-\mathrm{CH}_{3}\right), 19.73\left(\mathrm{~d},{ }^{4} \mathrm{~J}_{\mathrm{PC}}=11.0 \mathrm{~Hz}, \mathrm{Mes}-\mathrm{CH}_{3}\right)$, $27.54\left(\mathrm{~d}, J_{\mathrm{PC}}=22.0 \mathrm{~Hz},{ }^{i} \operatorname{Pr}-\mathrm{CH}\right), 119.71(\mathrm{Ar}-\mathrm{CH}), 126.8(\mathrm{Ar}-\mathrm{C}), 128.58(\mathrm{Ar}-C), 155.83(\operatorname{Ar}-C)$. ${ }^{31} \mathrm{P}\left\{{ }^{1} \mathrm{H}\right\}$ NMR (D8-THF, $\left.295 \mathrm{~K}\right): \delta(\mathrm{ppm}) 73.29$ (s). ${ }^{7} \mathrm{Li}$ NMR (D $\left.-\mathrm{THF}, 295 \mathrm{~K}\right): \delta(\mathrm{ppm})-1.40$ (s). FTIR (Nujol): ṽ (cm-1) 447 (w), 564 (s), 611 (m), 660 (m), 782 (s), 857 (m), 906 (s), 956 (m), 1007 (m), $1155(\mathrm{~s}), 1223(\mathrm{~s}), 1289(\mathrm{~s}), 1414(\mathrm{~m}), 1605(\mathrm{w})$.

\section{Preparation of [Li(DippNPPh $\left.\left.{ }_{2}\right)\left(\mathrm{OEt}_{2}\right)_{2}\right](\mathrm{S} 3)$}

${ }^{n} \operatorname{BuLi}(9 \mathrm{~mL}, 2.5 \mathrm{M}$ in hexanes, $22 \mathrm{mmol})$ was added to a pre-cooled $\left(-78{ }^{\circ} \mathrm{C}\right)$ solution of $\operatorname{DippN}(\mathrm{H}) \mathrm{PPh}_{2}(7.18 \mathrm{~g}, 19.9 \mathrm{mmol})$ in $\mathrm{Et}_{2} \mathrm{O}(75 \mathrm{~mL})$. The resulting yellow solution was allowed to warm slowly to room temperature and stirred for 3 hours. The reaction mixture was concentrated in vacuo and cooled to $-30{ }^{\circ} \mathrm{C}$ to afford $\left[\mathrm{Li}\left(\mathrm{DippNPPh}_{2}\right)\right]$ as a colourless crystalline solid. Colourless crystals of $\mathbf{S 3}$ suitable for a single crystal X-ray diffraction study were obtained from storage of a concentrated $\mathrm{Et}_{2} \mathrm{O}$ solution at $-30{ }^{\circ} \mathrm{C}$ for 72 hours. Yield: $7.38 \mathrm{~g}, 72 \%$. Anal. Calc'd for $\mathrm{C}_{32} \mathrm{H}_{47} \mathrm{LiNO}_{2} \mathrm{P}: \mathrm{C}, 74.54 ; \mathrm{H}, 9.19 ; \mathrm{N}, 2.72 \%$. Found: C, 74.38; H, 9.21; N, $2.76 \%$. ${ }^{1} \mathrm{H}$ NMR $\left(\mathrm{C}_{6} \mathrm{D}_{6}\right.$, $295 \mathrm{~K}): \delta(\mathrm{ppm}) 0.98\left(12 \mathrm{H}, \mathrm{t}, J_{\mathrm{HH}}=8.0 \mathrm{~Hz}, \mathrm{Et}_{2} \mathrm{O}-\mathrm{CH}_{3}\right), 1.16\left(12 \mathrm{H}, \mathrm{m},{ }^{i} \mathrm{Pr}-\mathrm{CH}_{3}\right), 3.18\left(8 \mathrm{H}, \mathrm{q}, \mathrm{J}_{\mathrm{HH}}=\right.$ $\left.8.0 \mathrm{~Hz}, \mathrm{Et}_{2} \mathrm{O}-\mathrm{CH}_{2}\right), 3.95$ (2H, m, $\left.{ }^{i} \mathrm{Pr}-\mathrm{CH}\right), 7,12(1 \mathrm{H}, \mathrm{m}, \mathrm{Ar}-\mathrm{CH}), 7.25$ (8H, m, Ar-CH), 7.78 (4H, m, Ar-CH). ${ }^{13} \mathrm{C}\left\{{ }^{1} \mathrm{H}\right\}$ NMR $\left(\mathrm{C}_{6} \mathrm{D}_{6}, 295 \mathrm{~K}\right): \delta(\mathrm{ppm}) 12.92\left(\mathrm{Et}_{2} \mathrm{O}-\mathrm{CH}_{3}\right), 22.58\left({ }^{i} \operatorname{Pr}-\mathrm{CH}_{3}\right), 25.53\left({ }^{i} \operatorname{Pr}-\mathrm{CH}\right)$, $63.53\left(\mathrm{Et}_{2} \mathrm{O}-\mathrm{CH}_{2}\right), 115.41(\mathrm{Ar}-\mathrm{CH}), 120.25(\mathrm{Ar}-\mathrm{CH}), 123.79(\mathrm{Ar}-\mathrm{CH}), 125.11(\mathrm{Ar}-\mathrm{CH}), 129.38$ (Ar-CH), 142.41 (Ar-C), 150.91(Ar-C), 154.69(Ar-C). ${ }^{31} \mathrm{P}\left\{{ }^{1} \mathrm{H}\right\}$ NMR $\left(\mathrm{C}_{6} \mathrm{D}_{6}, 295 \mathrm{~K}\right): \delta(\mathrm{ppm}) 43.54$ (br, s). ${ }^{7} \mathrm{Li} \mathrm{NMR}\left(\mathrm{C}_{6} \mathrm{D}_{6}, 295 \mathrm{~K}\right): \delta(\mathrm{ppm}) 1.17$ (s). FTIR (Nujol): $\tilde{\mathrm{v}}\left(\mathrm{cm}^{-1}\right) .511(\mathrm{~m}), 554(\mathrm{w}), 599$ 
(w), $736(\mathrm{~m}), 747(\mathrm{~m}), 782(\mathrm{~m}), 820(\mathrm{w}), 1000(\mathrm{w}), 1026(\mathrm{w}), 1052(\mathrm{w}), 1091(\mathrm{~m}), 1149(\mathrm{w}), 1194$ (m), $1238(\mathrm{~m}), 1311(\mathrm{~m}), 1421(\mathrm{~m}), 1433(\mathrm{~m}), 1585(\mathrm{w})$.

\section{Preparation of [Li(DippNPiPr 2$)]($ S4)}

${ }^{\mathrm{n}} \mathrm{BuLi}(3.3 \mathrm{~mL}, 2.5 \mathrm{M}$ in hexanes, $8.3 \mathrm{mmol})$ was added to a pre-cooled $\left(-78{ }^{\circ} \mathrm{C}\right)$ solution of $\operatorname{DippN}(\mathrm{H}) \mathrm{P}^{i} \operatorname{Pr}_{2}(2.21 \mathrm{~g}, 7.5 \mathrm{mmol})$ in $\mathrm{Et}_{2} \mathrm{O}(75 \mathrm{~mL})$. The resulting yellow solution was allowed to warm slowly to room temperature and stirred for 3 hours. The reaction mixture was concentrated in vacuo and cooled to $-30{ }^{\circ} \mathrm{C}$ to afford $\mathbf{S} 4$ as a colourless crystalline solid. Despite repeated attempts crystals suitable for single crystal X-ray diffraction study could not be obtained. Yield: $2.26 \mathrm{~g}, 81$ \%. Anal. Calc'd for $\mathrm{C}_{18} \mathrm{H}_{31}$ LiNP: C, 72.22; H, 10.44; N, 4.68\%. Found: C, 72.12; H, 10.32; N, 4.58 \%. ${ }^{1} \mathrm{H}$ NMR (D $\left.-\mathrm{THF}, 295 \mathrm{~K}\right): \delta(\mathrm{ppm}) 0.98\left(6 \mathrm{H}, \mathrm{m}, i \mathrm{Pr}-\mathrm{CH}_{3}\right), 1.11\left(18 \mathrm{H}, \mathrm{m}, i \mathrm{Pr}-\mathrm{CH}_{3}\right), 1.48(2 \mathrm{H}$, sept, $\left.{ }^{2} J_{\mathrm{HH}}=8.0 \mathrm{~Hz},{ }^{i} \operatorname{Pr}-\mathrm{CH}\right), 4.20\left(2 \mathrm{H}, \mathrm{m},{ }^{i} \operatorname{Pr}-\mathrm{CH}\right), 6.42\left(1 \mathrm{H}, \mathrm{t},{ }^{2} J_{\mathrm{HH}}=8.0 \mathrm{~Hz}, \mathrm{Ar}-\mathrm{CH}\right), 6.75(2 \mathrm{H}, \mathrm{d}$, $\left.{ }^{2} J_{\mathrm{HH}}=8.0 \mathrm{~Hz}, \mathrm{Ar}-\mathrm{CH}\right) .{ }^{13} \mathrm{C}\left\{{ }^{1} \mathrm{H}\right\} \mathrm{NMR}\left(\mathrm{D}_{8}-\mathrm{THF}, 295 \mathrm{~K}\right): \delta(\mathrm{ppm}) 16.52\left(\mathrm{~d}, J_{\mathrm{PC}}=13.0 \mathrm{~Hz},{ }^{i} \operatorname{Pr}-\mathrm{CH}_{3}\right)$, $17.14\left(\mathrm{~d}, J_{\mathrm{PC}}=19.0 \mathrm{~Hz},{ }^{i} \mathrm{Pr}-\mathrm{CH}_{3}\right), 24.64\left(\mathrm{~d}, J_{\mathrm{PC}}=8.0 \mathrm{~Hz},{ }^{i} \mathrm{Pr}-\mathrm{CH}\right), 27.49\left(\mathrm{~d}, J_{\mathrm{PC}}=23.0 \mathrm{~Hz},{ }^{i} \mathrm{Pr}-C \mathrm{H}\right)$, $113.53(\mathrm{Ar}-\mathrm{CH}), 120.74(\mathrm{Ar}-\mathrm{CH}), 140.54\left(\mathrm{~d},{ }^{3} J_{\mathrm{PC}}=4.0 \mathrm{~Hz}, \mathrm{Ar}-\mathrm{C}\right), 155.26\left(\mathrm{~d},{ }^{2} J_{\mathrm{PC}}=13.0 \mathrm{~Hz} \mathrm{Ar}-C\right)$. ${ }^{31} \mathrm{P}\left\{{ }^{1} \mathrm{H}\right\}$ NMR (D $\left.-\mathrm{THF}, 295 \mathrm{~K}\right): \delta(\mathrm{ppm}) 84.69$ (s). ${ }^{7} \mathrm{Li}$ NMR (D $\left.-\mathrm{THF}, 295 \mathrm{~K}\right): \delta(\mathrm{ppm})-1.50$ (s).

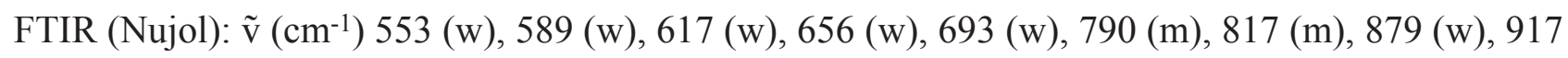
(m), $1041(\mathrm{w}), 1101(\mathrm{w}), 1143(\mathrm{w}), 1185(\mathrm{w}), 1233(\mathrm{w}), 1309(\mathrm{~m}), 1421(\mathrm{~m})$.

\section{Preparation of [Li(TrippNPPh $\left.)_{2}\left(\mathrm{OEt}_{2}\right)_{2}\right](\mathrm{S} 5)$}

${ }^{n} \mathrm{BuLi}(8.5 \mathrm{~mL}, 2.5 \mathrm{M}$ in hexanes, $21 \mathrm{mmol})$ was added to a pre-cooled $\left(-78{ }^{\circ} \mathrm{C}\right)$ solution of TrippN(H)PPh $2(7.48 \mathrm{~g}, 18.5 \mathrm{mmol})$ in $\mathrm{Et}_{2} \mathrm{O}(75 \mathrm{~mL})$. The resulting yellow solution was allowed to warm slowly to room temperature and stirred for 3 hours. The reaction mixture was concentrated in vacuo and cooled to $-30{ }^{\circ} \mathrm{C}$ to afford $\mathbf{S 5}$ as a colourless crystalline solution. Colourless crystals of S5 suitable for a single crystal X-ray diffraction study were obtained from storage of a concentrated $\mathrm{Et}_{2} \mathrm{O}$ solution at $-30{ }^{\circ} \mathrm{C}$ for 72 hours. Yield: $7.01 \mathrm{~g}, 68 \%$. Anal. Calc'd for $\mathrm{C}_{35} \mathrm{H}_{53} \mathrm{LiNO}_{2} \mathrm{P}: \mathrm{C}$, 
75.37; H, 9.58; N, $2.51 \%$. Found: C, 75.14; H, 9.38; N, $2.42 \% .{ }^{1} \mathrm{H}$ NMR $\left(\mathrm{C}_{6} \mathrm{D}_{6}, 295 \mathrm{~K}\right): \delta(\mathrm{ppm})$ $1.07\left(12 \mathrm{H}, \mathrm{t}, J_{\mathrm{HH}}=8.0 \mathrm{~Hz}, \mathrm{Et}_{2} \mathrm{O}-\mathrm{CH}_{3}\right), 1.29\left(12 \mathrm{H}, \mathrm{m},{ }^{i} \mathrm{Pr}-\mathrm{CH}_{3}\right), 1.31\left(6 \mathrm{H}, \mathrm{m},{ }^{i} \mathrm{Pr}-\mathrm{CH}_{3}\right), 2.87(1 \mathrm{H}, \mathrm{m}$, $\left.{ }^{i} \operatorname{Pr}-\mathrm{CH}\right), 3.24\left(8 \mathrm{H}, \mathrm{q}, J_{\mathrm{HH}}=8.0 \mathrm{~Hz}, \mathrm{Et}_{2} \mathrm{O}-\mathrm{CH}_{2}\right), 4.08\left(2 \mathrm{H}, \mathrm{m},{ }^{i} \mathrm{Pr}-\mathrm{CH}\right), 7.00(2 \mathrm{H}, \mathrm{s}, \mathrm{Ar}-\mathrm{CH}), 7.15$ (4H, m, Ar-CH), $7.17(2 \mathrm{H}, \mathrm{m}, \mathrm{Ar}-\mathrm{CH}), 7.71(4 \mathrm{H}, \mathrm{m}, \mathrm{Ar}-\mathrm{CH}) .{ }^{13} \mathrm{C}\left\{{ }^{1} \mathrm{H}\right\} \mathrm{NMR}\left(\mathrm{C}_{6} \mathrm{D}_{6}, 295 \mathrm{~K}\right): \delta$ (ppm) $22.63\left({ }^{i} \operatorname{Pr}-\mathrm{CH}_{3}\right), 25.29\left({ }^{i} \operatorname{Pr}-\mathrm{CH}\right) 32.22\left({ }^{i} \operatorname{Pr}-\mathrm{CH}\right), 117.98(\mathrm{Ar}-\mathrm{CH}), 123.67(\mathrm{Ar}-\mathrm{CH}), 125.03$ (Ar-CH), $129.40(\mathrm{Ar}-\mathrm{CH}), 135.06$ (Ar-C), 142.08 (Ar-C), 151.23 (Ar-C), 152.38 (Ar-C). ${ }^{31} \mathrm{P}\left\{{ }^{1} \mathrm{H}\right\}$ $\operatorname{NMR}\left(\mathrm{C}_{6} \mathrm{D}_{6}, 295 \mathrm{~K}\right): \delta(\mathrm{ppm}) 61.83$ (s). ${ }^{7} \mathrm{Li}$ NMR $\left(\mathrm{C}_{6} \mathrm{D}_{6}, 295 \mathrm{~K}\right): \delta(\mathrm{ppm}) 0.78$ (s). FTIR (Nujol): $\tilde{\mathrm{v}}$ (cm-1). $797(\mathrm{~m}), 841(\mathrm{w}), 876(\mathrm{w}), 924(\mathrm{w}), 950(\mathrm{~m}), 1032(\mathrm{w}), 1051(\mathrm{~m}), 1065(\mathrm{w}), 1102(\mathrm{w}), 1116$ (w), $1174(\mathrm{~m}), 1211(\mathrm{~m}), 1301(\mathrm{~m}), 1322(\mathrm{w}), 1358(\mathrm{w}), 1435(\mathrm{~m})$.

\section{Preparation of [Li(TrippNPiPr 2$\left.)(\mathrm{THF})_{2}\right](\mathrm{S} 6)$ and $\left[\mathrm{Li}_{2}\left(\mathrm{TrippNP}^{\mathrm{P}} \mathrm{Pr}_{2}\right)_{2}\left(\mathrm{Et}_{2} \mathrm{O}\right)_{2}\right](\mathrm{S} 7)$}

${ }^{\mathrm{n}} \mathrm{BuLi}\left(3.3 \mathrm{~mL}, 2.5 \mathrm{M}\right.$ in hexanes, $8.3 \mathrm{mmol}$ was added to a pre-cooled $\left(-78{ }^{\circ} \mathrm{C}\right)$ solution of $\operatorname{TrippN}(\mathrm{H}) \mathrm{P}^{i} \mathrm{Pr}_{2}(2.52 \mathrm{~g}, 7.5 \mathrm{mmol})$ in $\mathrm{Et}_{2} \mathrm{O}(75 \mathrm{~mL})$. The resulting yellow solution was allowed to warm slowly to room temperature and stirred for 3 hours. The reaction mixture was concentrated in vacuo and cooled to $-30{ }^{\circ} \mathrm{C}$ to afford $\left[\mathrm{Li}\left(\operatorname{TrippNP} \operatorname{Pr}_{2}\right)\right]$ as a colourless crystalline solid. Colourless crystals of S6 and S7 suitable for single crystal X-ray diffraction studies were obtained from storage of concentrated $\mathrm{THF}$ and $\mathrm{Et}_{2} \mathrm{O}$ solutions, respectively, at $-30{ }^{\circ} \mathrm{C}$ for 72 hours. Yield: $2.26 \mathrm{~g}, 88 \%$. Anal. Calc'd for $\mathrm{C}_{21} \mathrm{H}_{37} \mathrm{LiNP}$ : C, 73.87; H, 10.92; N, $4.10 \%$. Found: C, 74.25; H, 11.02; N, $4.45 \%$. ${ }^{1} \mathrm{H}$ NMR (D 8 -THF, $\left.295 \mathrm{~K}\right): \delta(\mathrm{ppm}) 0.99$ (6H, m, $\left.{ }^{i} \mathrm{Pr}-\mathrm{CH}_{3}\right), 1.06-1.20\left(24 \mathrm{H}, \mathrm{m},{ }^{i} \mathrm{Pr}-\mathrm{CH}_{3}\right), 1.47(2 \mathrm{H}$, sept, $\left.{ }^{2} J_{\mathrm{HH}}=6.0 \mathrm{~Hz},{ }^{i} \mathrm{Pr}-\mathrm{CH}\right) 2.68\left(1 \mathrm{H}, \mathrm{sept},{ }^{2} J_{\mathrm{HH}}=6.0 \mathrm{~Hz},{ }^{i} \mathrm{Pr}-\mathrm{CH}\right), 4.22\left(2 \mathrm{H}, \mathrm{m},{ }^{i} \mathrm{Pr}-\mathrm{CH}\right), 6.67(2 \mathrm{H}$, s, Ar-CH). ${ }^{13} \mathrm{C}\left\{{ }^{1} \mathrm{H}\right\}$ NMR $\left(\mathrm{D}_{8}-\mathrm{THF}, 295 \mathrm{~K}\right): \delta(\mathrm{ppm}) 18.61\left({ }^{i} \mathrm{Pr}-\mathrm{CH}_{3}\right), 19.09\left({ }^{i} \mathrm{Pr}-\mathrm{CH}_{3}\right), 26.51\left({ }^{i} \mathrm{Pr}-\right.$ CH), $29.46\left({ }^{i} \mathrm{Pr}-\mathrm{CH}\right), 33.88\left({ }^{i} \mathrm{Pr}-\mathrm{CH}\right) 120.35(\mathrm{Ar}-\mathrm{CH}), 134.85(\mathrm{Ar}-\mathrm{C}), 142.16(\mathrm{Ar}-\mathrm{C}), 154.66$ (ArC). ${ }^{31} \mathrm{P}\left\{{ }^{1} \mathrm{H}\right\}$ NMR (D $\left.-\mathrm{THF}, 295 \mathrm{~K}\right): \delta$ (ppm) 85.78 (s). ${ }^{7} \mathrm{Li}$ NMR $\left(\mathrm{D}_{8}\right.$-THF), $\left.295 \mathrm{~K}\right): \delta$ (ppm) 0.49 (s). FTIR (Nujol): ̃̃ (cm-1). 700 (m), 736 (m), 843 (w), 878 (w), 912 (w), 927 (w), 1088 (m), 1176 (m), $1211(\mathrm{~m}), 1305(\mathrm{w}), 1434(\mathrm{~m})$. 


\section{Preparation of [ClU(MesNPiPr $\left.\left.{ }_{2}\right)_{3}\right]$ and $\left[\mathrm{ClU}\left(\mathrm{MesNP}^{i} \mathrm{Pr}_{2}\right)_{3}\left\{\mu-\mathrm{LiCl}(\mathrm{THF})_{3}\right\}\right]$ (S8)}

THF $(30 \mathrm{ml})$ was added slowly to a cold $\left(-78^{\circ} \mathrm{C}\right)$ stirring mixture of $\mathrm{UCl}_{4}(0.38 \mathrm{~g}, 1.0 \mathrm{mmol})$ and S2 $(0.99 \mathrm{~g}, 1.5 \mathrm{mmol})$. The resultant mixture was allowed to warm to room temperature slowly and stirred over 16 hours. Volatiles were removed in vacuo and the resulting pale brown solid was extracted into hexane to afford $\mathbf{S 8}$ as light brown needles. Extraction of $\mathbf{S 8}$ into toluene, followed by filtration yielded salt-free [ClU(MesNPiPr$\left.)_{3}\right]$. Yield: 0.59 g, $59 \%$. Anal. Calc'd for $\mathrm{C}_{45} \mathrm{H}_{75} \mathrm{ClN}_{3} \mathrm{P}_{3} \mathrm{U}: \mathrm{C}, 52.76 ; \mathrm{H}, 7.38 ; \mathrm{N}, 4.10 \%$. Found: $\mathrm{C}, 52.72 ; \mathrm{H}, 7.42 ; \mathrm{N}, 3.96 \%$ \%. ${ }^{1} \mathrm{H}$ NMR $\left(\mathrm{C}_{6} \mathrm{D}_{6}, 295 \mathrm{~K}\right): \delta(\mathrm{ppm})-4.42\left(12 \mathrm{H}, \mathrm{s}-\mathrm{br},{ }^{i} \mathrm{Pr}-\mathrm{CH}_{3}\right),-2.22\left(18 \mathrm{H}, \mathrm{s},{ }^{i} \mathrm{Pr}-\mathrm{CH}_{3}\right), 4.24\left(6 \mathrm{H}, \mathrm{s},{ }^{i} \mathrm{Pr}-\mathrm{CH}\right)$, $6.75\left(9 \mathrm{H}, \mathrm{s}, \mathrm{Mes}-\mathrm{CH}_{3}\right), 7.35\left(18 \mathrm{H}, \mathrm{s}, \mathrm{Mes}-\mathrm{CH}_{3}\right), 13.18(6 \mathrm{H}, \mathrm{s}, \mathrm{Ar}-\mathrm{CH}) .{ }^{31} \mathrm{P}\left\{{ }^{1} \mathrm{H}\right\} \mathrm{NMR}\left(\mathrm{C}_{6} \mathrm{D}_{6}, 295\right.$ K): $\delta(\mathrm{ppm})$ 1949. FTIR (Nujol): $\tilde{\mathrm{v}}\left(\mathrm{cm}^{-1}\right) 529(\mathrm{w}), 568(\mathrm{w}), 590(\mathrm{w}), 655(\mathrm{w}), 666(\mathrm{w}), 759(\mathrm{w})$, $853(\mathrm{w}), 880(\mathrm{w}), 902(\mathrm{w}), 927(\mathrm{w}), 961(\mathrm{w}), 1055(\mathrm{w}), 1153(\mathrm{~m}), 1213(\mathrm{~m}), 1302(\mathrm{~m})$. Magnetic moment (Evans method, $\mathrm{C}_{6} \mathrm{D}_{6}, 298 \mathrm{~K}$ ): $\mu_{\mathrm{eff}}=2.27 \mu_{\mathrm{B}}$.

\section{Preparation of $\left[\mathrm{ClTh}\left(\mathrm{MesNPi}^{\mathrm{P}} \mathrm{r}_{2}\right)_{3}\right](\mathrm{S} 9)$}

THF $(30 \mathrm{ml})$ was added slowly to a cold $\left(-78^{\circ} \mathrm{C}\right)$ stirring mixture of $\mathrm{ThCl}_{4}(\mathrm{DME})_{2}(0.55 \mathrm{~g}, 1.0$ mmol) and [Li(MesNPiPr 2$)](0.77 \mathrm{~g}, 3.0 \mathrm{mmol})$, the resultant mixture was allowed to warm to room temperature slowly and stirred over 16 hours. Volatiles were removed in vacuo and the resulting pale yellow solid was extracted into hexane to afford $\mathbf{S 9}$ as an analytically pure pale yellow solid. Colourless crystals of $\mathbf{S 9}$ suitable for a single crystal X-ray diffraction study were obtained from storage of a hexane solution at $-30{ }^{\circ} \mathrm{C}$ for 72 hours. Yield: $0.93 \mathrm{~g}, 91 \%$. Anal. Calc'd for $\mathrm{C}_{45} \mathrm{H}_{75} \mathrm{ClN}_{3} \mathrm{P}_{3}$ Th: $\mathrm{C}, 53.07 ; \mathrm{H}, 7.42 ; \mathrm{N}, 4.13 \%$. Found: C, 52.40; H, 7.35; N, $3.95 \%$ \%. ${ }^{1} \mathrm{H}$ NMR $\left(\mathrm{C}_{6} \mathrm{D}_{6}, 295 \mathrm{~K}\right): \delta(\mathrm{ppm}) 0.98\left(18 \mathrm{H}, \mathrm{m},{ }^{i} \mathrm{Pr}-\mathrm{CH}_{3}\right), 1.21\left(18 \mathrm{H}, \mathrm{m},{ }^{i} \mathrm{Pr}-\mathrm{CH}_{3}\right), 2.20\left(9 \mathrm{H}, \mathrm{s}, \mathrm{Mes}-\mathrm{CH}_{3}\right)$, $2.30\left(6 \mathrm{H}, \mathrm{m},{ }^{\mathrm{i}} \mathrm{Pr}-\mathrm{CH}\right), 2.46\left(18 \mathrm{H}, \mathrm{m}, \mathrm{Mes}-\mathrm{CH}_{3}\right), 6.79(6 \mathrm{H}, \mathrm{s}, \mathrm{Mes}-\mathrm{CH}) .{ }^{13} \mathrm{C}\left\{{ }^{1} \mathrm{H}\right\} \mathrm{NMR}\left(\mathrm{C}_{6} \mathrm{D}_{6}, 295\right.$ $\mathrm{K}): \delta(\mathrm{ppm}) 19.60\left({ }^{i} \mathrm{Pr}-\mathrm{CH}_{3}\right), 20.41\left({ }^{i} \mathrm{Pr}-\mathrm{CH}_{3}\right), 21.47\left(\mathrm{Mes}-\mathrm{CH}_{3}\right), 29.60\left({ }^{i} \mathrm{Pr}-\mathrm{CH}\right), 129.10(\mathrm{Ar}-\mathrm{CH})$, 131.08 (Ar-C), 132.00 (Ar-C), $150.12\left(\right.$ Ar-C). ${ }^{31} \mathrm{P}\left\{{ }^{1} \mathrm{H}\right\}$ NMR $\left(\mathrm{C}_{6} \mathrm{D}_{6}, 295 \mathrm{~K}\right): \delta(\mathrm{ppm}) 54.15$ (s). 


\section{Preparation of [IU(MesNPiPr $\left.)_{3}\right](\mathrm{S} 10)$}

To a cooled $\left(0^{\circ} \mathrm{C}\right)$ solution of $\left[\mathrm{ClU}\left(\mathrm{MesNP}^{\mathrm{i}} \mathrm{Pr}_{2}\right)_{3}\right](2.56 \mathrm{~g}, 2.5 \mathrm{mmol})$ in toluene $(30 \mathrm{ml})$ was added $\mathrm{Me}_{3} \mathrm{SiI}(0.63 \mathrm{~g}, 0.45 \mathrm{ml}, 3.13 \mathrm{mmol})$. The resulting brown solution was allowed to warm slowly to room temperature and stirred for 18 hours to afford a brown solution. Volatiles were removed in vacuo and the resulting pale brown solid was extracted into hexane and cooled $\left(-30{ }^{\circ} \mathrm{C}\right)$ to afford S10 as green blocks over 24 h. Yield: 1.56 g, 56\%. Anal. Calc'd for $\mathrm{C}_{45} \mathrm{H}_{75} \mathrm{IN}_{3} \mathrm{P}_{3} \mathrm{U} 0.2\left(\mathrm{C}_{6} \mathrm{H}_{14}\right)$ : C, 48.97; H, 6.92; N, $3.71 \%$. Found: C, 49.24; H, 7.07; N, $3.74 \% .{ }^{1} \mathrm{H}$ NMR $\left(\mathrm{C}_{6} \mathrm{D}_{6}, 295 \mathrm{~K}\right): \delta(\mathrm{ppm})$ $5.98\left(18 \mathrm{H}, \mathrm{s}-\mathrm{br}, \mathrm{CH}_{3}\right), 1.96\left(18 \mathrm{H}, \mathrm{s}, \mathrm{CH}_{3}\right), 2.62\left(6 \mathrm{H}, \mathrm{s},{ }^{i} \operatorname{Pr}-\mathrm{CH}\right), 6.45\left(9 \mathrm{H}, \mathrm{s}, \mathrm{CH}_{3}\right), 9.40(18 \mathrm{H}, \mathrm{s}$, $\left.\mathrm{CH}_{3}\right), 12.77(6 \mathrm{H}, \mathrm{s}, \mathrm{Ar}-\mathrm{CH}) .{ }^{31} \mathrm{P}\left\{{ }^{1} \mathrm{H}\right\} \operatorname{NMR}\left(\mathrm{C}_{6} \mathrm{D}_{6}, 295 \mathrm{~K}\right): \delta(\mathrm{ppm}) 2106$. FTIR (Nujol): $\tilde{\mathrm{v}}(\mathrm{cm}-1)$ $519(\mathrm{~m}), 531(\mathrm{~s}), 569(\mathrm{w}), 591(\mathrm{~m}), 662(\mathrm{~s}), 681(\mathrm{~m}), 760(\mathrm{~s}), 852(\mathrm{~s}), 901(\mathrm{~s}), 925(\mathrm{~m}), 959(\mathrm{~m})$, 1037 (m), 1053 (s), 1153 (s), 1205 (s), 1218 (s), 1301 (m), 1366 (m). Magnetic moment (Evans method, $\left.\mathrm{C}_{6} \mathrm{D}_{6}, 298 \mathrm{~K}\right): \mu_{\mathrm{eff}}=2.40 \mu_{\mathrm{B}}$.

\section{Preparation of [ITh $\left.\left(\mathrm{MesNP}^{\mathrm{P}} \mathrm{Pr}_{2}\right)_{3}\right](\mathrm{S} 11)$}

To a cooled $\left(0{ }^{\circ} \mathrm{C}\right)$ solution of $\mathbf{S 9}(0.51 \mathrm{~g}, 0.5 \mathrm{mmol})$ in hexane $(30 \mathrm{ml})$ was added $\mathrm{Me}_{3} \mathrm{SiI}(0.11 \mathrm{~g}$, $0.08 \mathrm{~mL} 0.55 \mathrm{mmol})$. The resulting pale yellow solution was allowed to warm slowly to room temperature and stirred for 18 hours to afford a colourless solution and a colourless precipitate. The precipitate was isolated by filtration and washed with hexane $(3 \times 10 \mathrm{~mL})$ to afford $\mathbf{S 1 1}$ as a colourless solid. Colourless crystals of $\mathbf{S 1 1}$ suitable for a single crystal X-ray diffraction study were obtained from storage of a ${ }^{\mathrm{n}} \mathrm{Bu}_{2} \mathrm{O}$ solution at $25^{\circ} \mathrm{C}$ for 24 hours. Yield: $0.45 \mathrm{~g}, 82 \%$. Anal. Calc'd for $\mathrm{C}_{45} \mathrm{H}_{75} \mathrm{IN}_{3} \mathrm{P}_{3}$ Th: $\mathrm{C}, 48.70 ; \mathrm{H}, 6.81 ; \mathrm{N}, 3.78 \%$. Found: C, 48.31; H, 6.75; N, $3.76 \%$. ${ }^{1} \mathrm{H}$ NMR $\left(\mathrm{C}_{6} \mathrm{D}_{6}, 295 \mathrm{~K}\right): \delta(\mathrm{ppm}) 1.00\left(18 \mathrm{H}, \mathrm{t},{ }^{2} J_{\mathrm{HH}}=8.0 \mathrm{~Hz},{ }^{i} \mathrm{Pr}-\mathrm{CH}_{3}\right), 1.22\left(18 \mathrm{H}, \mathrm{m},{ }^{i} \mathrm{Pr}-\mathrm{CH}_{3}\right), 2.19(9 \mathrm{H}, \mathrm{s}$, Mes- $\left.\mathrm{CH}_{3}\right), 2.36\left(6 \mathrm{H}, \mathrm{sept},{ }^{2} \mathrm{~J}_{\mathrm{HH}}=7.0 \mathrm{~Hz},{ }^{i} \mathrm{Pr}-\mathrm{CH}\right), 2.44\left(18 \mathrm{H}, \mathrm{m}, \mathrm{Mes}-\mathrm{CH}_{3}\right), 6.77(6 \mathrm{H}, \mathrm{s}, \mathrm{Mes}-\mathrm{CH})$. ${ }^{13} \mathrm{C}\left\{{ }^{1} \mathrm{H}\right\}$ NMR $\left(\mathrm{C}_{6} \mathrm{D}_{6}, 295 \mathrm{~K}\right): \delta(\mathrm{ppm}) 19.82\left({ }^{i} \operatorname{Pr}-\mathrm{CH}_{3}\right), 20.40\left({ }^{i} \mathrm{Pr}-\mathrm{CH}_{3}\right), 21.64\left(\mathrm{Mes}-\mathrm{CH}_{3}\right), 30.90$ ( $\left.{ }^{i} \operatorname{Pr}-C H\right), 131.30\left(\right.$ Ar-CH), $131.10($ Ar- $C), 131.80(\operatorname{Ar}-C), 149.92(\operatorname{Ar}-C) .{ }^{31} \mathrm{P}\left\{{ }^{1} \mathrm{H}\right\}$ NMR $\left(\mathrm{C}_{6} \mathrm{D}_{6}, 295\right.$ 
K): $\delta(\mathrm{ppm}) 50.41(\mathrm{~s})$. FTIR v/cm-1 (Nujol): $660(\mathrm{w}), 683(\mathrm{w}), 761(\mathrm{w}), 852(\mathrm{w}), 883(\mathrm{w}), 900(\mathrm{w})$, $924(w), 958(w), 1036(w), 1053(w), 1152(w), 1216(m)$.

\section{Preparation of $\left[\mathrm{Cl}_{3} \mathrm{U}\left(\mathrm{DippNPPh}_{2}\right)_{2}\right]\left[\mathrm{Li}(\mathrm{THF})_{4}\right](\mathrm{S} 12)$}

THF $(30 \mathrm{ml})$ was added slowly to a cold $\left(-78^{\circ} \mathrm{C}\right)$ stirring mixture of $\mathrm{UCl}_{4}(0.19 \mathrm{~g}, 0.5 \mathrm{mmol})$ and $\mathbf{S 3}(0.76 \mathrm{~g}, 1.5 \mathrm{mmol})$, the resultant mixture was allowed to warm to room temperature slowly and stirred over 16 hours. The resulting brown reaction mixture was concentrated to $5 \mathrm{ml}$ and cooled to $-30{ }^{\mathrm{O}} \mathrm{C}$ to afford $\mathbf{S 1 2}$ as a brown crystalline solid. Yield $0.35 \mathrm{~g}, 52 \%$. Anal. Calc'd for $\mathrm{C}_{64} \mathrm{H}_{86} \mathrm{Cl}_{3} \mathrm{~N}_{2} \mathrm{P}_{2} \mathrm{O} 4 \mathrm{LiU} \cdot \mathrm{OC}_{4} \mathrm{H}_{8}:$ C, 56.98; H, 6.62; N, $1.96 \%$. Found: C, 56.72; H, 6.43; N, $1.91 \%$. ${ }^{1} \mathrm{H}$ NMR $\left(\mathrm{C}_{6} \mathrm{D}_{6}, 295 \mathrm{~K}\right): \delta(\mathrm{ppm})-43.57,-31.45,-8.03,4.23,6.87,8.21,8.82,10.25,11.79 .13 .47$, 17.17, 21.69. FTIR (Nujol): ṽ (cm-1) 646 (m), 698 (m), 744 (m), 771 (m), 890 (w), 923 (w), 1042 (m), $1100(\mathrm{~m}), 1156(\mathrm{~m}), 1188(\mathrm{~m}), 1240(\mathrm{~m}), 1321(\mathrm{w})$. Magnetic moment (Evans method, $\mathrm{C}_{6} \mathrm{D}_{6}$, $298 \mathrm{~K}): \mu_{\mathrm{eff}}=2.05 \mu_{\mathrm{B}}$

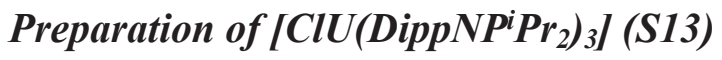

THF $(30 \mathrm{ml})$ was added slowly to a cold $\left(-78^{\circ} \mathrm{C}\right)$ stirring mixture of $\mathrm{UCl}_{4}(0.38 \mathrm{~g}, 1.0 \mathrm{mmol})$ and S4 $(1.12 \mathrm{~g}, 3.0 \mathrm{mmol})$, the resultant mixture was allowed to warm to room temperature slowly and stirred over 16 hours. Volatiles were removed in vacuo and the resulting pale brown solid was extracted into hexane, concentrated to $15 \mathrm{ml}$ and cooled to $-30{ }^{\circ} \mathrm{C}$ to afford $\mathbf{S 1 3}$ as a light brown crystalline solid. Brown needles of $\mathbf{S 1 3}$ suitable for a single crystal X-ray diffraction study were obtained from storage of a hexane solution at room temperature ${ }^{\circ} \mathrm{C}$ for 72 hours. Yield $0.56 \mathrm{~g}, 49$ \%. Anal. Calc'd for $\mathrm{C}_{54} \mathrm{H}_{93} \mathrm{ClN}_{3} \mathrm{P}_{3} \mathrm{U}$ : C, 56.36; H, 8.15; N, 3.65 \%. Found: C, 56.15; H, 7.98; N, $3.46 \% .{ }^{1} \mathrm{H}$ NMR $\left(\mathrm{C}_{6} \mathrm{D}_{6}, 295 \mathrm{~K}\right): \delta(\mathrm{ppm})-30.33(6 \mathrm{H}, \mathrm{s}-\mathrm{br}, \mathrm{CH}),-22.5(3 \mathrm{H}, \mathrm{s}-\mathrm{br}, \mathrm{Ar}-\mathrm{CH}) 9.95(36 \mathrm{H}$, s-br, CH3), 16.11 (36H, s-br, CH3), 26.27 (6H, s-br, CH), 35.57 (6H, s-br, CH). FTIR (Nujol): $\tilde{v}$ (cm-1) $561(\mathrm{w}), 670(\mathrm{w}), 722(\mathrm{w}), 768(\mathrm{w}), 877(\mathrm{w}), 892(\mathrm{w}), 904(\mathrm{w}), 904(\mathrm{w}), 915(\mathrm{w}), 928(\mathrm{w})$, 
$960(w), 1019(w), 1041(w), 1180(w), 1232(w), 1307(w), 1322(w), 1336(w), 1365(w), 1426$

(m). Magnetic moment (Evans method, $\mathrm{C}_{6} \mathrm{D}_{6}, 298 \mathrm{~K}$ ): $\mu_{\mathrm{eff}}=2.58 \mu_{\mathrm{B}}$.

\section{Preparation of $\left[\mathrm{Cl}_{3} \mathrm{U}\left(\mathrm{TrippNPPh}_{2}\right)_{2}\right]\left[\mathrm{Li}(\mathrm{THF})_{4}\right](\mathrm{S} 14)$}

THF $(30 \mathrm{ml})$ was added slowly to a cold $\left(-78^{\circ} \mathrm{C}\right)$ stirring mixture of $\mathrm{UCl}_{4}(0.19 \mathrm{~g}, 0.5 \mathrm{mmol})$ and S7 $(0.62 \mathrm{~g}, 0.75 \mathrm{mmol})$, the resultant mixture was allowed to warm to room temperature slowly and stirred over 16 hours. The resulting brown reaction mixture was concentrated to $5 \mathrm{ml}$ and cooled to $-30{ }^{\circ} \mathrm{C}$ to afford $\mathbf{S 1 4}$ as a brown crystalline solid. Yield $0.32 \mathrm{~g}, 44 \%$. Anal. Calc'd for $\mathrm{C}_{70} \mathrm{H}_{98} \mathrm{Cl}_{3} \mathrm{~N}_{2} \mathrm{P}_{2} \mathrm{O}_{4} \mathrm{LiU}: \mathrm{C}, 58.19 ; \mathrm{H}, 6.84 ; \mathrm{N}, 1.94 \%$. Found: $\mathrm{C}, 61.00 ; \mathrm{H}, 7.16 ; \mathrm{N}, 2.22 \%$. ${ }^{1} \mathrm{H}$ NMR $\left(\mathrm{C}_{6} \mathrm{D}_{6}, 295 \mathrm{~K}\right): \delta(\mathrm{ppm})-43.11,-31.60,-8.03,4.15,4.20,4.27,6.62,6.78,8.26,8.83,10.32,13.65$, 17.15, 21.72. FTIR (Nujol): $\tilde{v}\left(\mathrm{~cm}^{-1}\right) 635$ (w), 644 (w), 659 (w), 698 (s), 733 (m), 747 (s), 793 (s), $835(\mathrm{w}), 874(\mathrm{~m}), 886(\mathrm{~m}), 913$ (m), 942 (m), 1042 (s), $1084(\mathrm{~m}), 1098(\mathrm{~m}), 1117(\mathrm{~m}), 1208(\mathrm{~m})$, $1314(\mathrm{~m}), 1338(\mathrm{~s})$. Magnetic moment (Evans method, $\left.\mathrm{C}_{6} \mathrm{D}_{6}, 298 \mathrm{~K}\right): \mu_{\mathrm{eff}}=2.23 \mu_{\mathrm{B}}$.

\section{X-ray Diffraction Data}

Crystals were examined using either a) an Agilent Supernova diffractometer, equipped with either an Atlas/AtlasS2 or TitanS2 CCD area detector and mirror-monochromated $\mathrm{Cu} \mathrm{K} \alpha$ radiation $(\lambda=$ $1.5418 \AA$ ) or b) Bruker SMART APEX diffractometer equipped with a CCD area detector with Mo $\mathrm{K} \alpha$ radiation $(\lambda=0.71073 \AA)$. Intensities were integrated from a sphere of data recorded on narrow $\left(0.5\right.$ or $\left.1.0^{\circ}\right)$ frames by $\omega$ rotation. Cell parameters were refined from the observed positions of all strong reflections in each data set. Either multi-scan or Gaussian grid face-indexed absorption corrections with a beam profile correction were applied. The structures were solved by direct methods using either SHELXS, ${ }^{1}$ dual methods using SHELXT, ${ }^{2}$ or iteratively with Olex2.solve, ${ }^{3}$ and the datasets were refined by full-matrix least-squares on all unique $F^{2}$ values, with anisotropic displacement parameters for all non-hydrogen atoms, and with constrained riding hydrogen geometries; $U_{\text {iso }}(\mathrm{H})$ was set at 1.2 (1.5 for methyl groups) times $U_{\text {eq }}$ of the parent atom. The largest 
features in final difference syntheses were close to heavy atoms and were of no chemical significance. CrysAlisPro ${ }^{4}$ and SMART APEX/SAINT-PLUS ${ }^{5}$ was used for control and integration, and SHELXL ${ }^{6}$ and Olex $2^{3}$ were employed for structure refinement. ORTEP-3 and POV-Ray were employed for molecular graphics. ${ }^{7,8}$ Some of the crystal structures reported here are structurally analogous to prior examples in the literature but with different substituent patterns. ${ }^{9-11}$

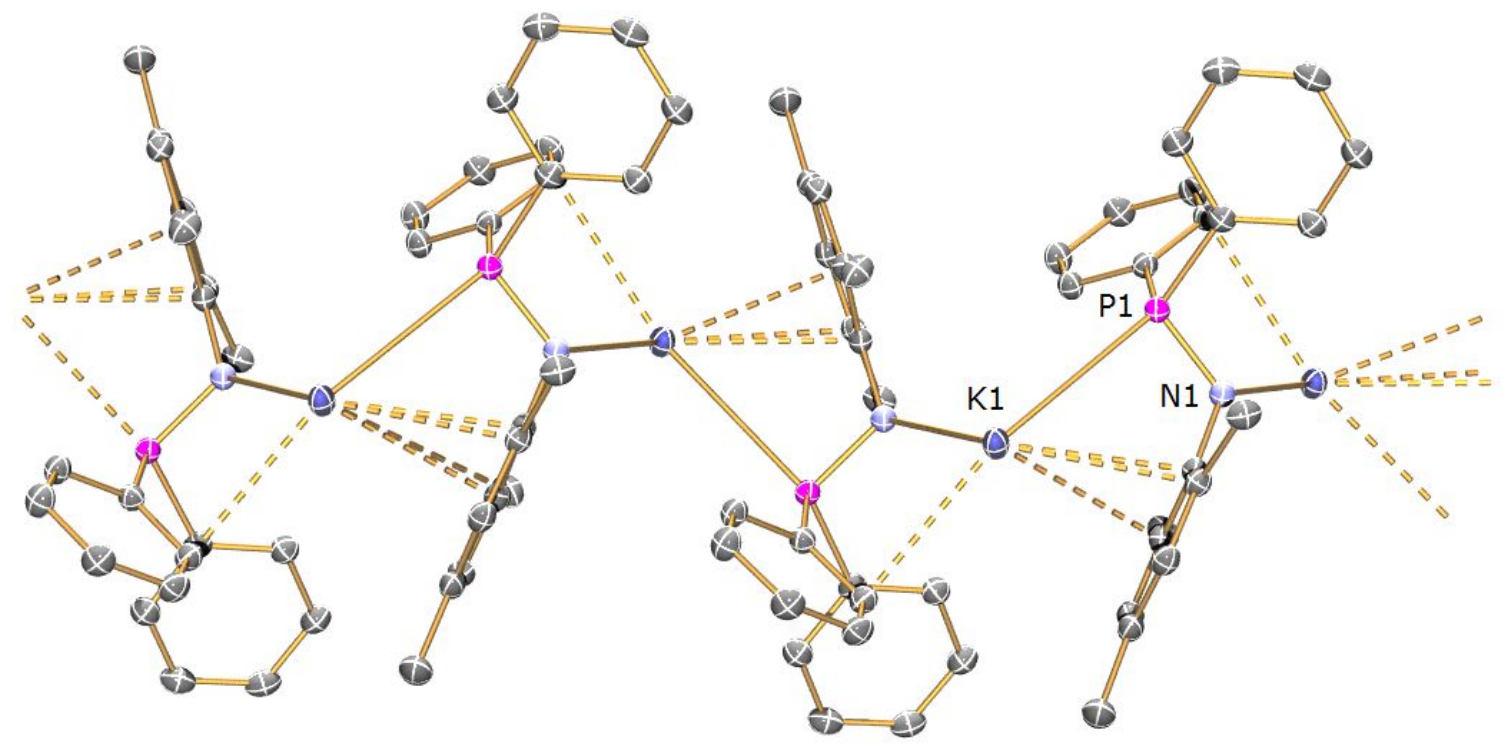

Figure S1. Solid state structure of complex S1 at $120 \mathrm{~K}$. Thermal ellipsoids are set at $30 \%$ probability and hydrogen atoms and lattice solvent are omitted for clarity. Selected bond lengths

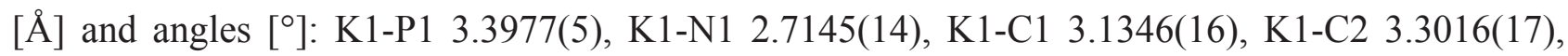
K1-C3 3.3982(19), K1-C4 3.4604(18), K1-C5 3.5006(17), K1-C7 3.3533(17), K1-C8 3.1920(17), K1-C16 3.3431(17), K1-C17 3.1273(18), K1-C25 3.416(11), P1-N1 1.6590(14), P1-C10 1.8524(17), P1-C16 1.8535(16), N1-C1 1.405(2), P1-K1-C3 87.50(4), P1-K1-C4 87.30(3), P1-K1C5 96.24(3), P1-K1-C25 93.87(13), N1-K1-P1 98.59(3), N1-K1-C1 118.57(4), N1-K1-C2 103.24(4), N1-K1-C3 54.72(4), N1-K1-C4 107.84(4), N1-K1-C5 126.97(5), N1-K1-C7 148.53(5), N1-K1-C8 144.34(4), N1-K1-C16 52.79(4), N1-K1-C17 73.11(4), N1-K1-C25 140.79(14), C1-K1P1 46.02(3), C2-K1-P1 64.64(3), C7-K1-P1 83.45(3), C8-K1-P1 59.05(3), C16-K1-P1 151.30(3), C17-K1-P1 160.47(4), C17-K1-C1 121.96(4). 

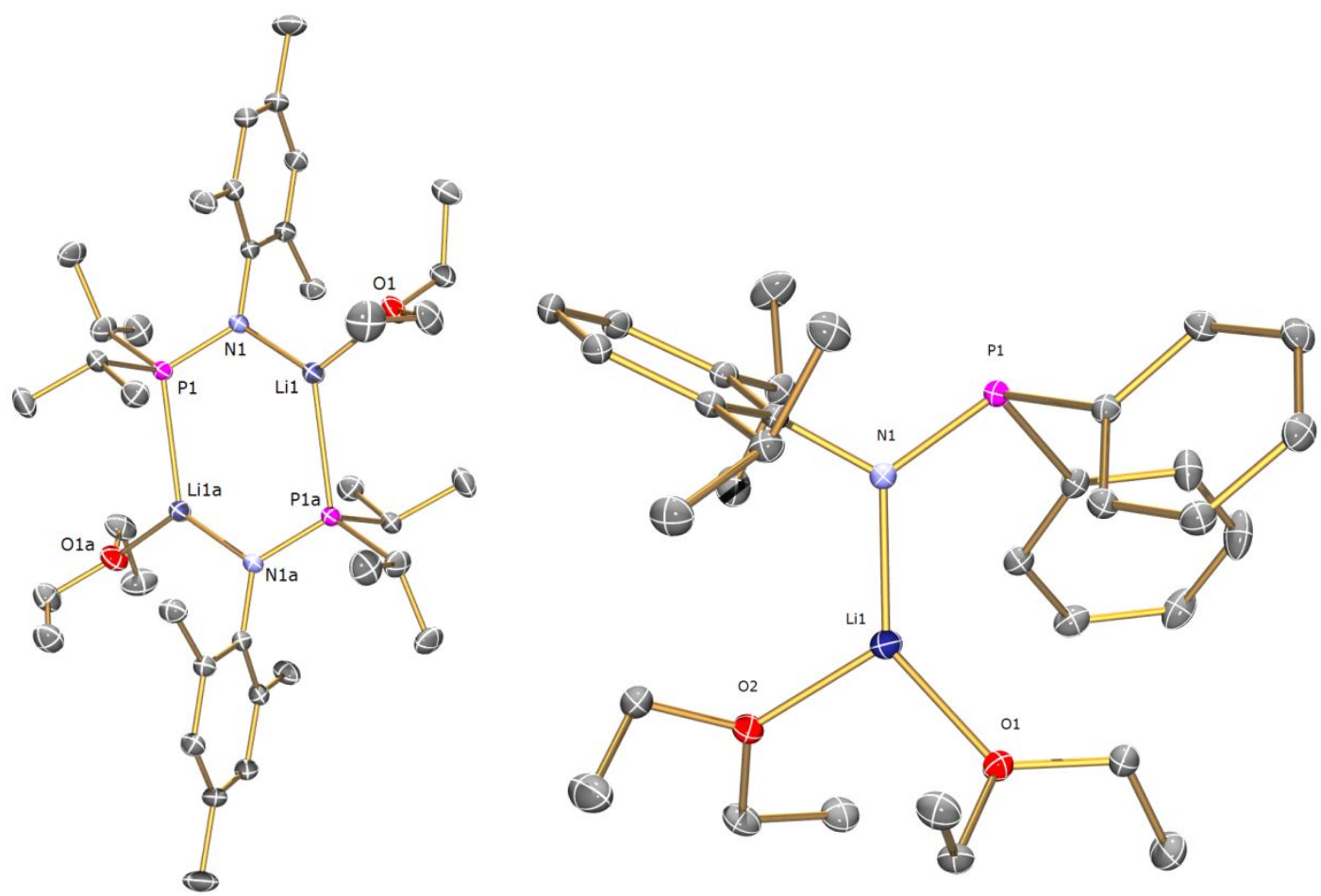

Figure S2. Solid state structure of complex S2 at 120K (left) and S3 at 90K (right). Thermal ellipsoids are set at 30\% probability and hydrogen atoms and disordered components are omitted for clarity. Selected bond lengths $[\AA]$ and angles $\left[{ }^{\circ}\right]$ for S2: P1-N1 1.6483(14), P1-Li1 2.591(3), O1C16 1.429(3), O1-C18 1.439(4), O1-Li1 1.934(4), O1A-Li1 1.975(9), N1-C1 1.415(2), N1-Li1 1.930(3), N1-P1-Li1 113.91(8), P1-N1-Li1 114.11(11), O2-Li2-P2 117.26(14), N2-Li2-P2 124.38(14), N2-Li2-O2 118.37(17). S3: P1-N1 1.6617(9), O1-C25 1.4420(13), O1-C27 1.4434(13), O1-Li1 1.9669(19), O2-C29 1.4402(13), O2-C31 1.4408(14), O2-Li1 1.946(2), N1-C1 1.4151(12), N1-Li1 1.956(2), O2-Li1-O1 104.18(9), O2-Li1-N1 121.15(10), N1-Li1-O1 132.50(10). 

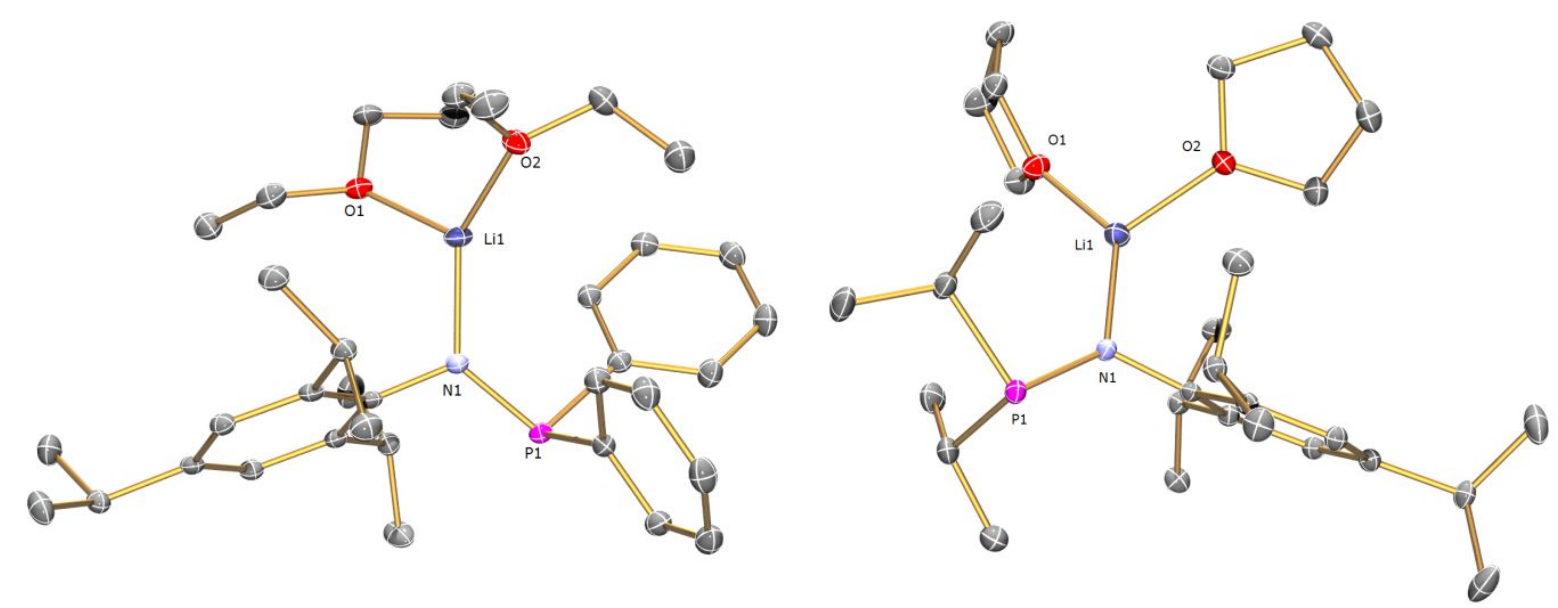

Figure S3. Solid state structures of S5 at 90K (left) and S6 at 120K (right). Selected bond lengths $[\AA]$ and angles $\left[^{\circ}\right]$ for S5: P1-N1 1.6654(12), O1-C28 1.442(2), O1-C30 1.442(2), O1-Li1 1.942(3), O2-C32 1.4415(19), O2-C34 1.442(2), O2-Li1 1.995(3), N1-Li1 1.943(3), N1-P1-C16 101.30(7), N1-P1-C22 106.83(7), C16-P1-C22 97.01(7), C28-O1-Li1 114.77(13), C30-O1-Li1 130.29(13), C32-O2-Li1 122.58(13), C34-O2-Li1 118.21(12), P1-N1-Li1 127.43(11), C1-N1-P1 117.02(9), C1N1-Li1 112.89(12). S6: P1-N1 1.6781(14), O1-Li1 1.934(3), O2-Li1 1.956(3), N1-C1 1.406(2), N1Li1 1.918(3), C1-Li1 2.740(3), N1-P1-C16 99.61(8), N1-P1-C19 106.27(8), C16-P1-C19 98.85(9), C22-O1-Li1 125.22(15), C25-O1-Li1 125.74(15), C26-O2-Li1 122.31(14), C29-O2-Li1 128.40(14), P1-N1-Li1 127.03(12), C1-N1-P1 121.70(11), C1-N1-Li1 110.03(14). 


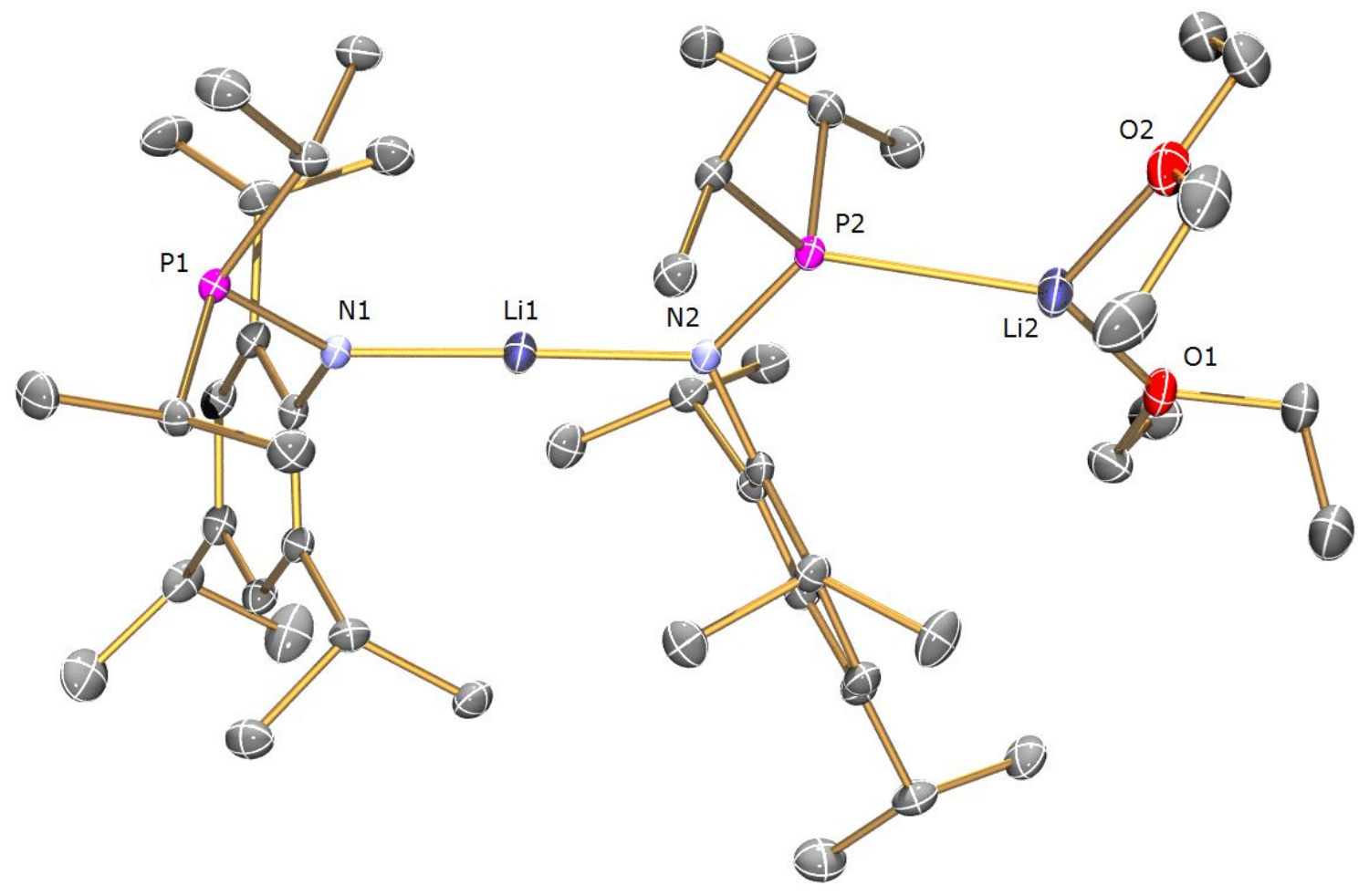

Figure S4. Solid state structure of complex S7 at 120K. Thermal ellipsoids are set at 30\% probability and hydrogen atoms are omitted for clarity. Selected bond lengths $[\AA]$ and angles $\left[^{\circ}\right]$ : P1-N1 1.6829(12), P2-N2 1.6612(13), P2-Li2 2.538(3), O1-Li2 1.879(3), O2-C48 1.444(3), O2C49 1.426(3), O2-Li2 1.949(3), N1-Li1 1.898(3), N2-Li1 1.923(3), N1-P1-C16 103.72(6), N1-P1C20 100.71(6), C20-P1-C16 102.15(7), N2-P2-C37 104.29(6), N2-P2-C40 109.32(7), N2-P2-Li2 125.78(8), C37-P2-C40 98.90(7), C37-P2-Li2 117.84(9), C40-P2-Li2 96.75(9), C43-O1-Li2 124.12(14), C45-O1-Li2 119.67(15), C48-O2-Li2 127.91(18), C49-O2-Li2 118.78(17), P1-N1-Li1 134.51(11), P2-N2-Li1 126.52(11), C22-N2-P2 116.83(9), C22-N2-Li1 115.83(12). 


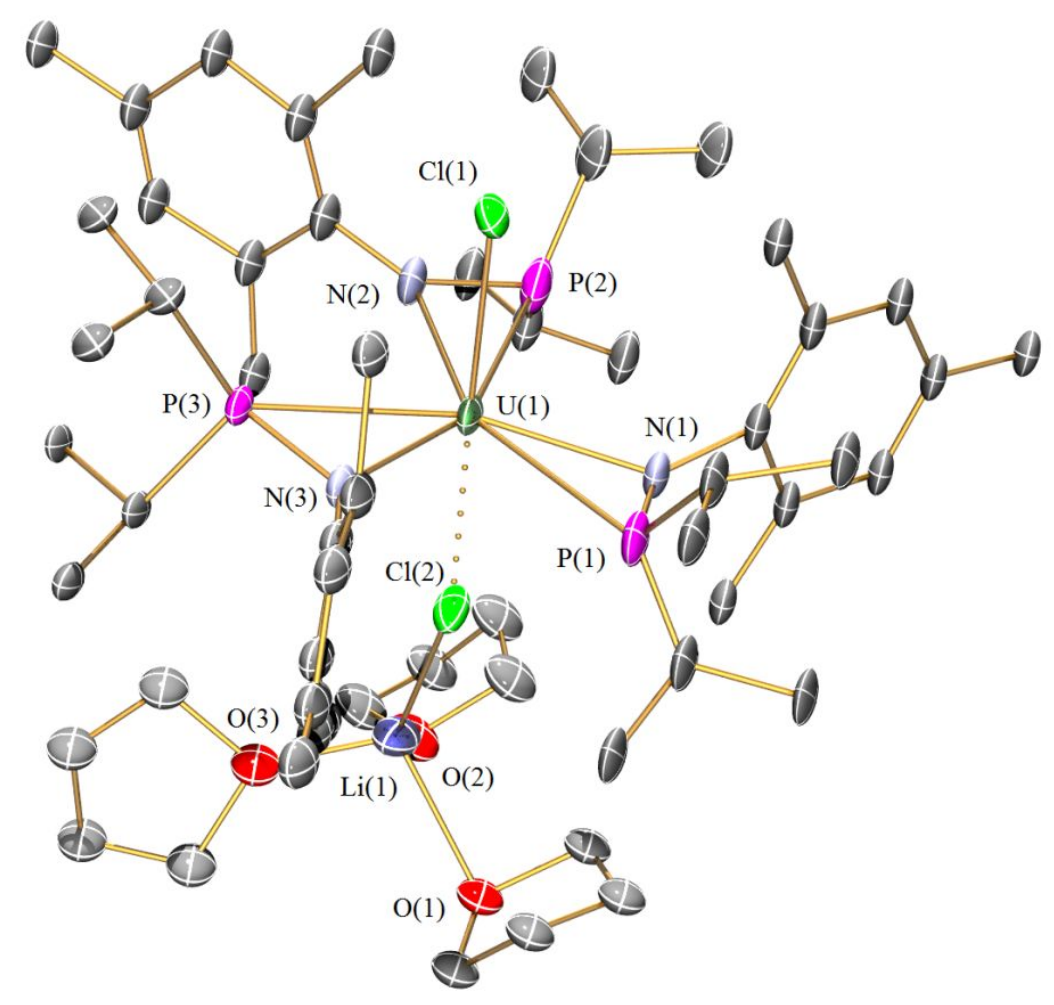

Figure S5. Solid state structure of complex S8 at 90K. Thermal ellipsoids are set at 30\% probability and hydrogen atoms, disordered components and lattice solvent are omitted for clarity. Selected bond lengths $[\AA]$ and angles $\left[^{\circ}\right]$ : U1-Cl1 2.701(11), U1-Cl2 2.793(11), U1-P1 2.945(13), U1-P2 2.905(9), U1-P3 2.916(9), U1-N1 2.38(3), U1-N2 2.38(3), U1-N3 2.38(3), Cl2-Li1 2.58(8), P1-N1 1.77(3), P2-N2 1.70(3), P3-N3 1.72(3), O1-Li1 2.05(3), O2-Li1 2.04(3), O3-Li1 2.04(3), Cl1-U1C12 179.3(4), C11-U1-P1 80.1(4), C11-U1-P2 98.6(6), C11-U1-P3 85.2(5), C12-U1-P1 99.7(3), Cl2U1-P2 80.8(6), Cl2-U1-P3 95.5(5), P2-U1-P1 120.7(3), P2-U1-P3 119.7(3), P3-U1-P1 119.2(3), N1-U1-C11 72.5(8), N1-U1-C12 107.8(8), N1-U1-P1 36.9(8), N1-U1-P2 156.0(8), N1-U1-P3 82.4(8), N2-U1-C11 99.3(14), N2-U1-Cl2 79.9(14), N2-U1-P1 85.4(8), N2-U1-P2 35.7(8), N2-U1P3 155.3(8), N2-U1-N1 122.2(11), N2-U1-N3 119.0(10), N3-U1-Cl1 92.1(14), N3-U1-Cl2 88.3(14), N3-U1-P1 155.3(7), N3-U1-P2 83.5(6), N3-U1-P3 36.3(6), N3-U1-N1 118.4(10), N1-P1U1 54.0(12), N1-P1-C13 113.6(16), C10-P1-U1 151(2), C10-P1-N1 112(2), C10-P1-C13 98(3), C13-P1-U1 110.8(14), N2-P2-U1 54.9(9), N2-P2-C25 111(2), N2-P2-C28 112(3), C25-P2-U1 118.7(16), C28-P2-U1 133.3(19), C28-P2-C25 107.9(19), N3-P3-U1 54.8(10), N3-P3-C40 113(2), N3-P3-C43 106(2), C40-P3-U1 129.4(17), C40-P3-C43 108.4(13), C43-P3-U1 122.2(14). 


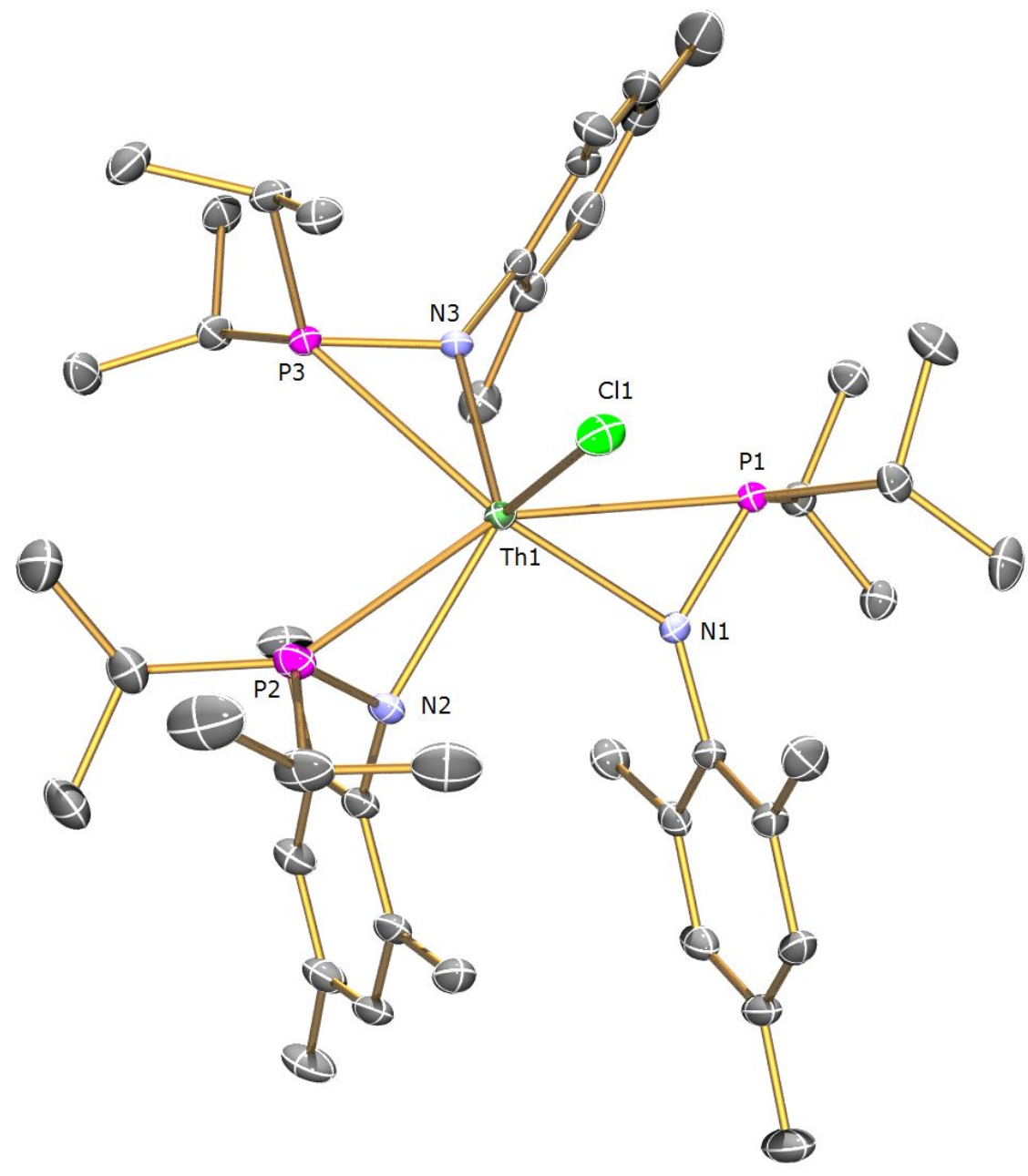

Figure S6. Solid state structure of complex S9 at 120K. Thermal ellipsoids are set at $30 \%$ probability and hydrogen atoms and lattice solvent are omitted for clarity. Selected bond lengths $[\AA]$ and angles $\left[{ }^{\circ}\right]$ : Th1-Cl1 2.6713(9), Th1-P1 2.8910(10), Th1-P2 2.9217(9), Th1-P3 2.9776(8), Th1-N1 2.391(3), Th1-N2 2.332(3), Th1-N3 2.346(3), P1-N1 1.680(3), P2-N2 1.684(3), P3-N3 1.689(3), C11-Th1-P1 94.53(3), C11-Th1-P2 85.55(3), Cl1-Th1-P3 109.54(3), P1-Th1-P2 150.96(3), P1-Th1-P3 86.18(3), P2-Th1-P3 121.20(2), N1-Th1-C11 119.37(8), N1-Th1-P1 35.51(8), N1-Th1P2 121.63(8), N1-Th1-P3 100.07(8), N2-Th1-Cl1 95.85(7), N2-Th1-P1 116.42(7), N2-Th1-P2 35.18(7), N2-Th1-P3 144.84(7), N2-Th1-N1 87.44(10), N2-Th1-N3 114.68(10), N3-Th1-Cl1 109.10(8), N3-Th1-P1 120.21(7), N3-Th1-P2 86.72(7), N3-Th1-P3 34.49(7), N3-Th1-N1 $124.03(11)$ 


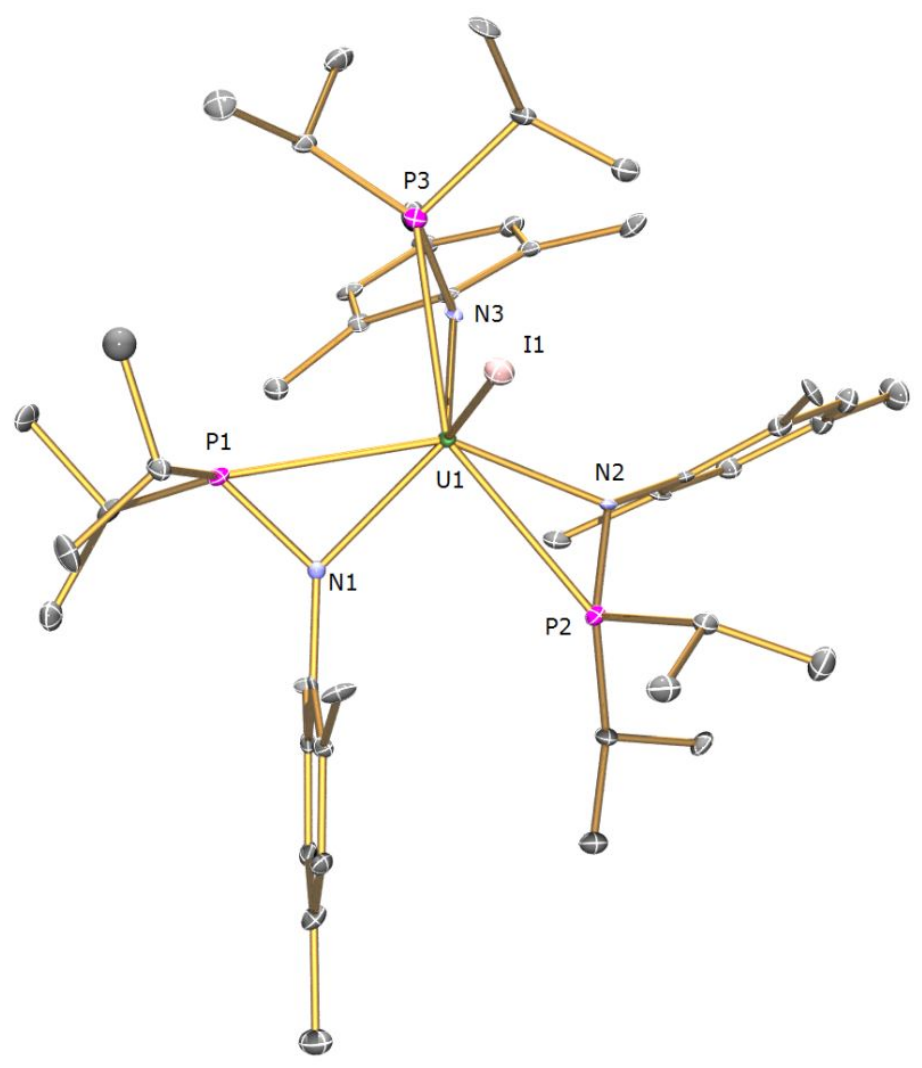

Figure S7. Solid state structure of complex S10 at 90K. Thermal ellipsoids are set at $30 \%$ probability and hydrogen atoms are omitted for clarity. Selected bond lengths $[\AA]$ and angles $\left[{ }^{\circ}\right]$ : U1-I1 3.0121(10), U1-P1 2.861(3), U1-P2 2.845(3), U1-P3 2.826(3), U1-N1 2.284(8), U1-N2 2.272(8), U1-N3 2.319(8), P1-N1 1.668(8), P2-N2 1.677(8), P3-N3 1.690(8), P1-U1-I1 94.47(6), P2-U1-I1 88.27(6), P2-U1-P1 118.84(8), P3-U1-I1 87.21(6), P3-U1-P1 93.13(8), P3-U1-P2 147.97(8), N1-U1-I1 101.29(19), N1-U1-P1 35.6(2), N1-U1-P2 83.9(2), N1-U1-P3 128.0(2), N1U1-N3 127.7(3), N2-U1-I1 101.3(2), N2-U1-P1 148.6(2), N2-U1-P2 36.1(2), N2-U1-P3 114.5(2), N2-U1-N1 113.8(3), N2-U1-N3 89.9(3), N3-U1-I1 119.61(18), N3-U1-P1 105.7(2), N3-U1-P2 125.2(2), N3-U1-P3 36.69(19). 


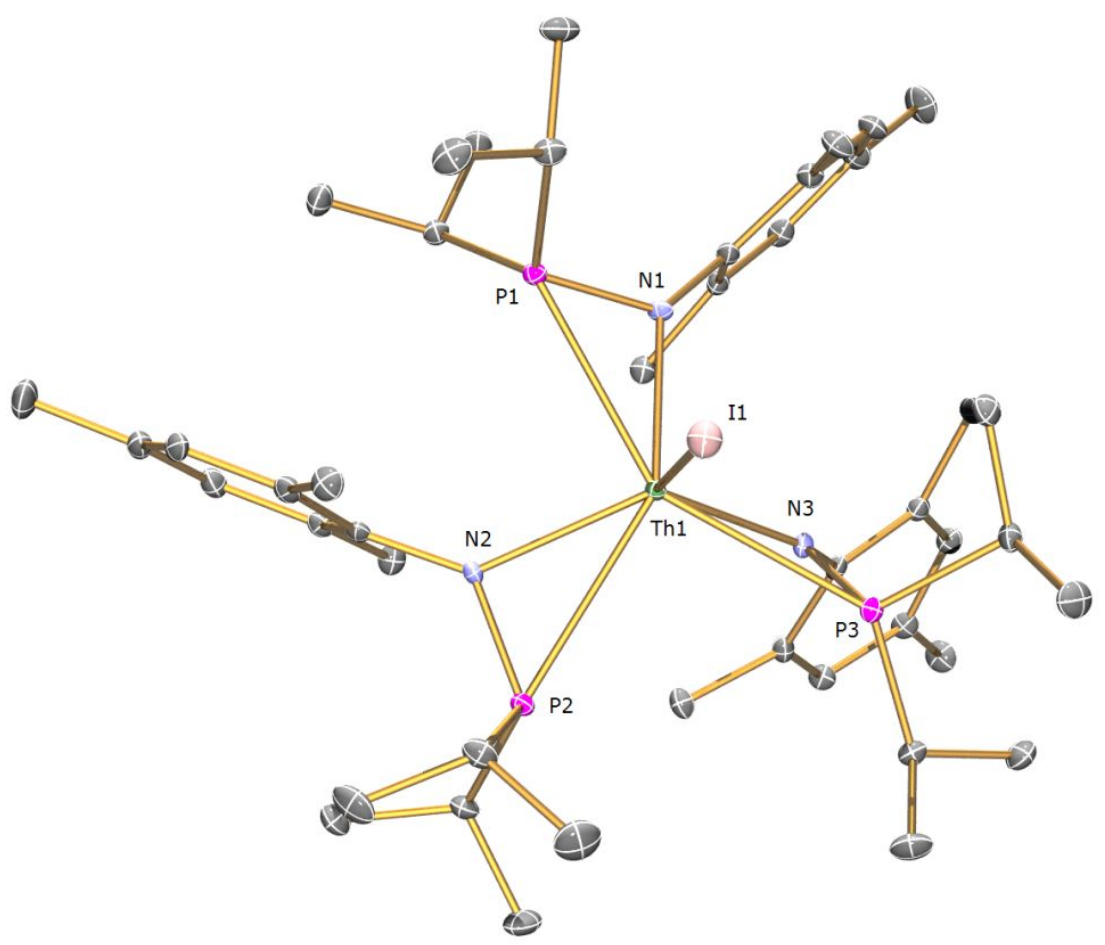

Figure S8. Solid state structure of complex S11 at 120K. Thermal ellipsoids are set at 30\% probability and hydrogen atoms are omitted for clarity. Selected bond lengths $[\AA]$ and angles $\left[{ }^{\circ}\right]$ : Th1-I1 3.0761(3), Th1-P1 2.9517(8), Th1-P2 2.8900(9), Th1-P3 2.9126(8), Th1-N1 2.337(3), Th1N2 2.384(3), Th1-N3 2.325(3), P1-N1 1.683(3), P2-N2 1.696(3), P3-N3 1.690(3), P1-Th1-I1 96.279(18), P2-Th1-I1 88.615(18), P2-Th1-P1 93.00(2), P2-Th1-P3 147.59(2), P3-Th1-I1 89.298(18), P3-Th1-P1 119.37(2), N1-Th1-I1 103.36(7), N1-Th1-P1 34.71(7), N1-Th1-P2 126.60(7), N1-Th1-P3 85.25(7), N1-Th1-N2 124.45(10), N2-Th1-I1 120.57(7), N2-Th1-P1 103.81(7), N2-Th1-P2 35.93(7), N2-Th1-P3 124.35(7), N3-Th1-I1 102.11(7), N3-Th1-P1 147.27(7), N3-Th1-P2 114.09(7), N3-Th1-P3 35.45(7), N3-Th1-N1 113.69(10), N3-Th1-N2 89.85(10). 


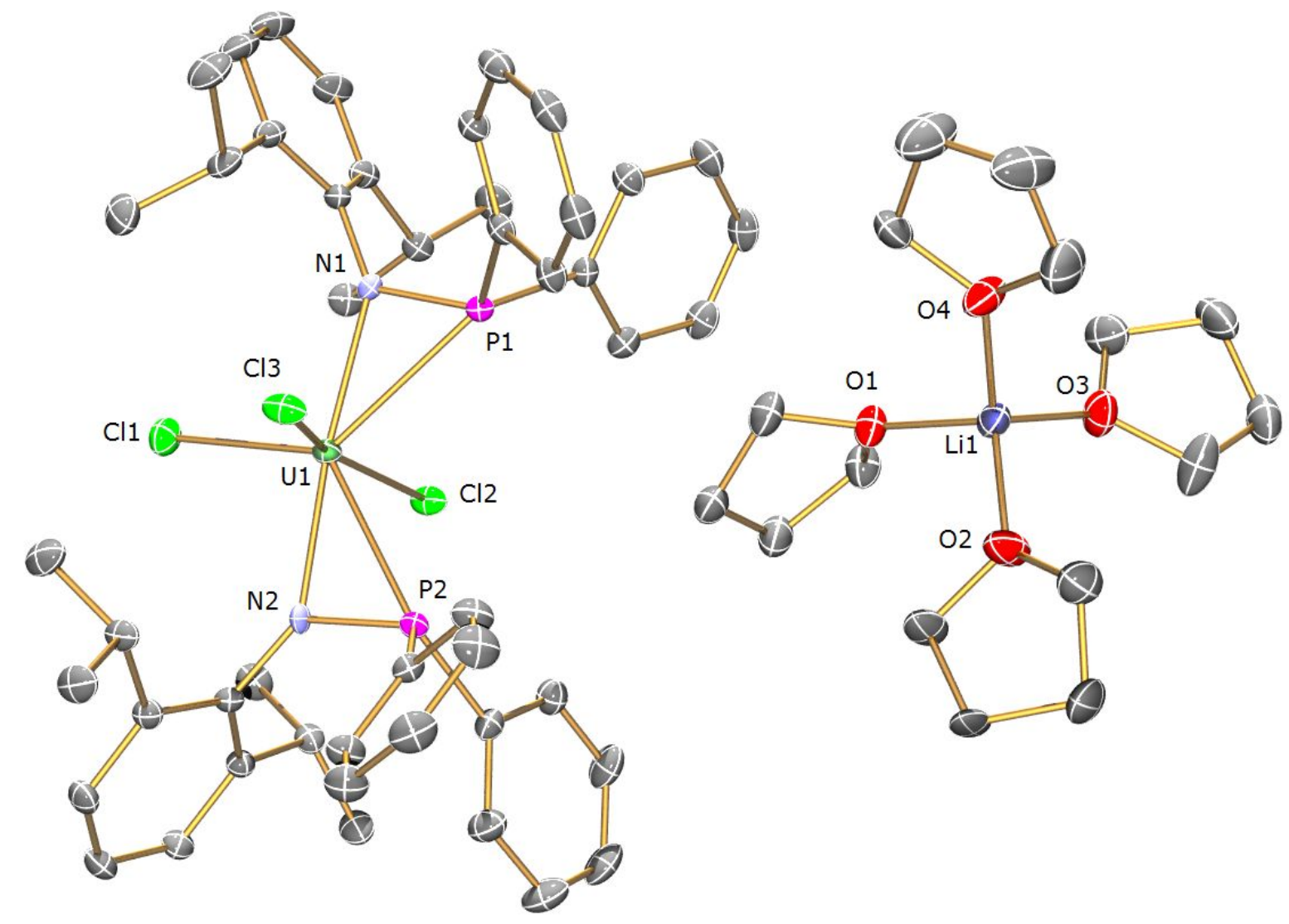

Figure S9. Solid state structure of complex S12 at 150K. Thermal ellipsoids are set at 30\% probability and hydrogen atoms, disordered components and lattice solvent are omitted for clarity. Selected bond lengths $[\AA]$ and angles $\left[^{\circ}\right]$ : U1-Cl1 2.6238(11), U1-Cl2 2.6503(10), U1-Cl3 2.6500(10), U1-P1 2.8410(10), U1-P2 2.8401(10), U1-N1 2.307(5), U1-N2 2.313(4), P1-N1 1.682(4), P2-N2 1.686(4), O1-Li1 1.922(9), O2-Li1 1.930(8), O3-Li1 1.905(8), O4-Li1 1.899(8), Cl1-U1-Cl2 108.57(4), Cl1-U1-Cl3 92.16(4), Cl1-U1-P1 129.33(3), Cl1-U1-P2 130.06(3), Cl2-U1P1 80.72(3), Cl2-U1-P2 81.03(3), Cl3-U1-Cl2 159.27(3), Cl3-U1-P1 85.85(3), Cl3-U1-P2 85.96(3), P2-U1-P1 100.34(3), N1-U1-Cl1 93.20(11), N1-U1-Cl2 88.39(11), N1-U1-Cl3 90.08(11), N1-U1P1 36.30(10), N1-U1-P2 136.63(10), N1-U1-N2 172.85(16), N2-U1-Cl1 93.87(11), N2-U1-C12 88.36(11), N2-U1-Cl3 90.70(11), N2-U1-P1 136.72(11), N2-U1-P2 36.40(11). 


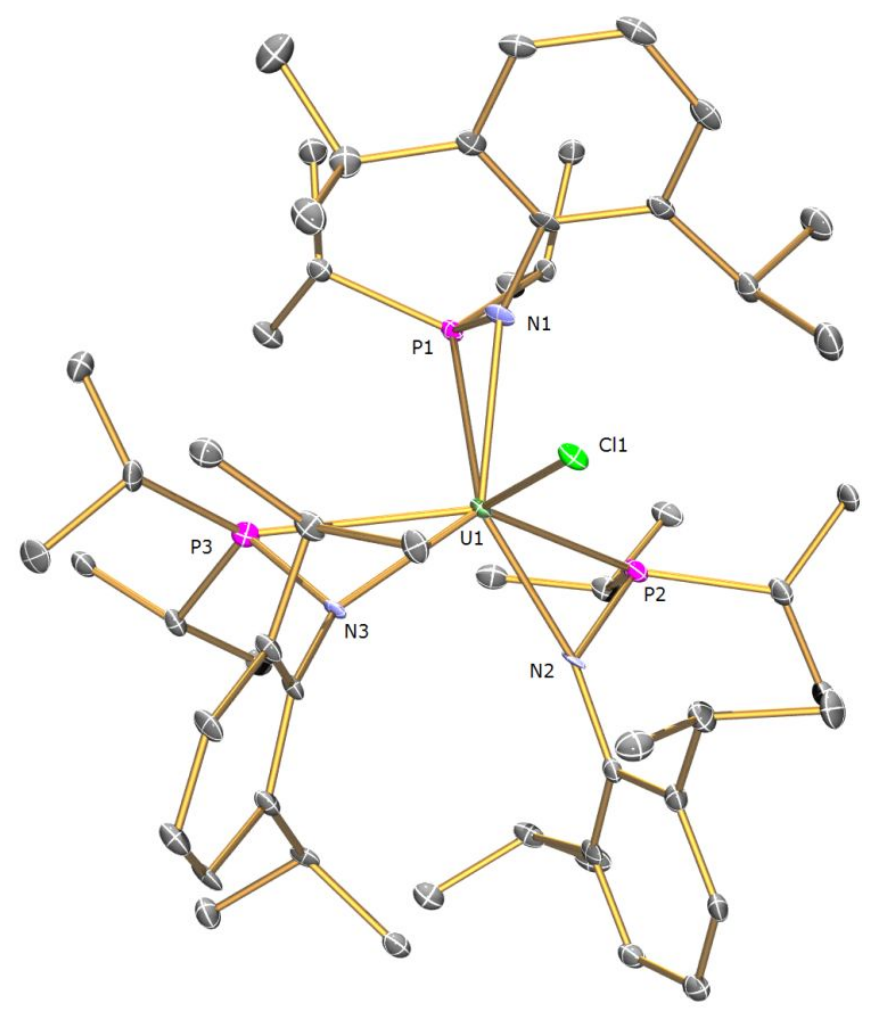

Figure S10. Solid state structure of complex S13 at 90K. Thermal ellipsoids are set at 30\% probability and hydrogen atoms are omitted for clarity. Selected bond lengths $[\AA]$ and angles $\left[{ }^{\circ}\right]$ : U1-C11 2.589(3), U1-P1 2.858(3), U1-P2 2.850(3), U1-P3 2.837(3), U1-N1 2.378(8), U1-N2 2.365(7), U1-N3 2.364(8), P1-N1 1.690(8), P2-N2 1.689(8), P3-N3 1.680(9), C11-U1-P1 112.63(8), Cl1-U1-P2 112.00(8), Cl1-U1-P3 113.85(8), P2-U1-P1 133.55(8), P3-U1-P1 83.63(8), P3-U1-P2 89.90(8), N1-U1-C11 109.2(2), N1-U1-P1 36.20(19), N1-U1-P2 114.68(19), N1-U1-P3 116.2(2), N2-U1-Cl1 90.2(2), N2-U1-P1 132.4(2), N2-U1-P2 36.3(2), N2-U1-P3 125.9(2), N2-U1-N1 97.8(3), N3-U1-C11 77.6(2), N3-U1-P1 99.3(2), N3-U1-P2 102.1(2), N3-U1-P3 36.3(2), N3-U1-N1 135.1(3), N3-U1-N2 127.0(3). 


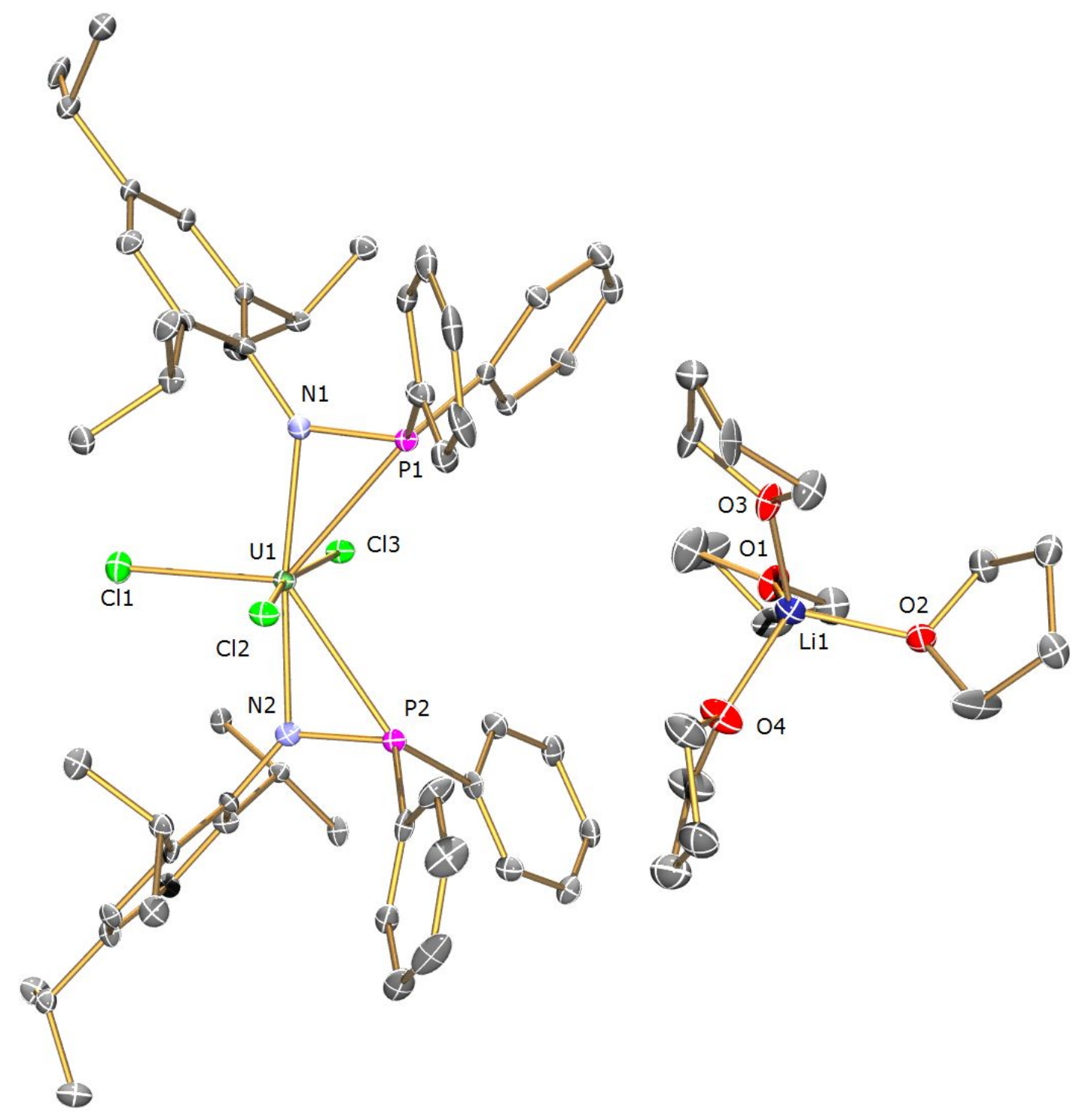

Figure S11. Solid state structure of complex S14 at 90K. Thermal ellipsoids are set at 30\% probability and hydrogen atoms and lattice solvent are omitted for clarity. Selected bond lengths

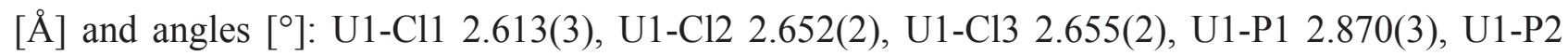
2.881(3), U1-N1 2.316(8), U1-N2 2.305(8), P1-N1 1.658(13), P2-N2 1.665(13), O1-Li1 1.918(19), O2-Li1 1.92(2), O3-Li1 1.94(3), O4-Li1 1.90(2), Cl1-U1-Cl2 102.78(9), Cl1-U1-Cl3 89.75(9), Cl1U1-P1 127.60(10), Cl1-U1-P2 129.61(10), Cl2-U1-C13 167.47(7), Cl2-U1-P1 81.89(10), Cl2-U1P2 81.80(10), Cl3-U1-P1 90.53(10), Cl3-U1-P2 90.23(10), P1-U1-P2 102.79(7), N1-U1-Cl1 92.3(3), N1-U1-Cl2 89.8(3), N1-U1-Cl3 89.7(3), N1-U1-P1 35.3(3), N1-U1-P2 138.1(3), N2-U1C11 94.3(3), N2-U1-Cl2 90.0(3), N2-U1-Cl3 89.0(3), N2-U1-P1 138.1(3), N2-U1-P2 35.3(3), N2U1-N1 173.2(3). 


\section{SQUID Data}
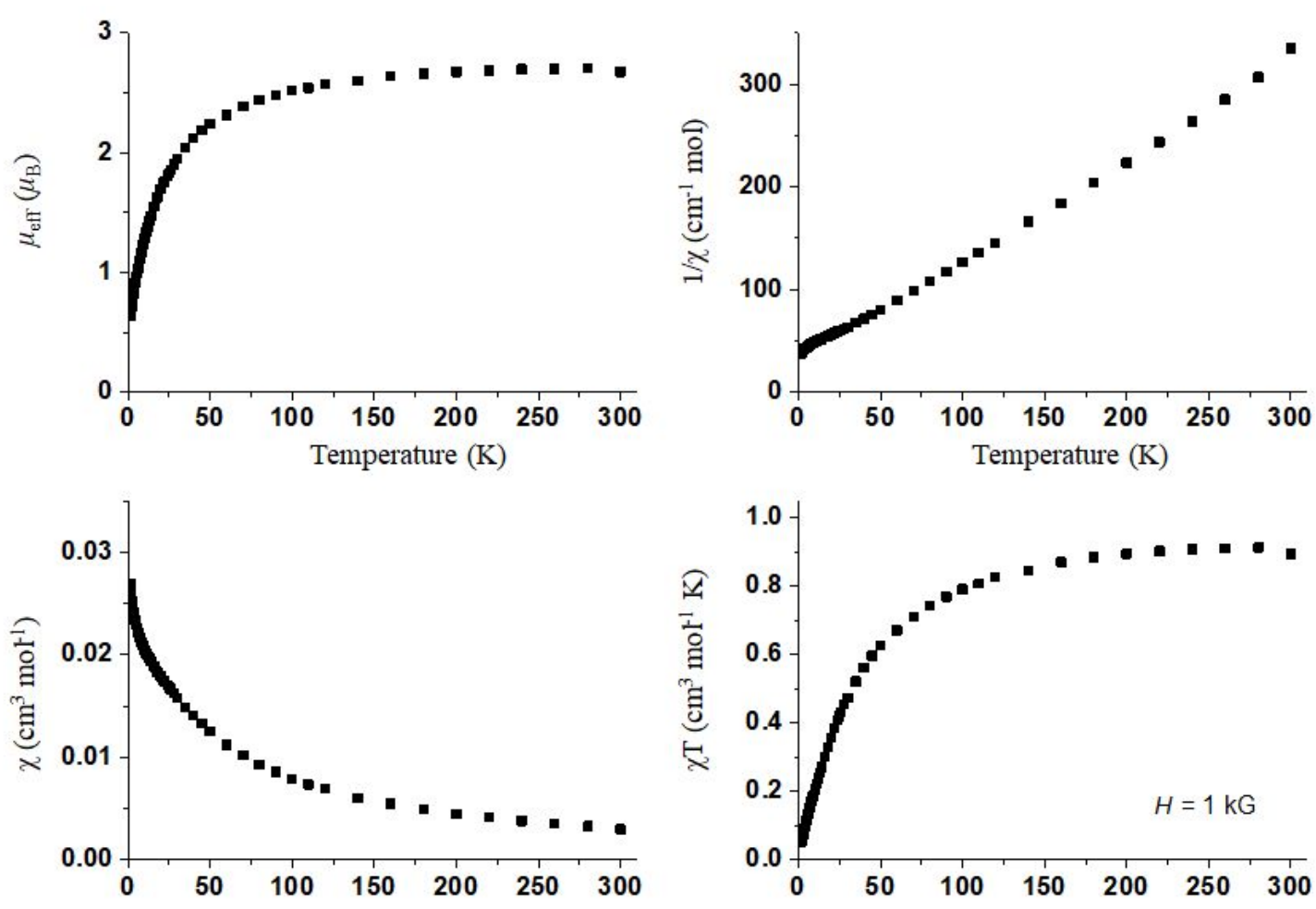

Temperature (K)

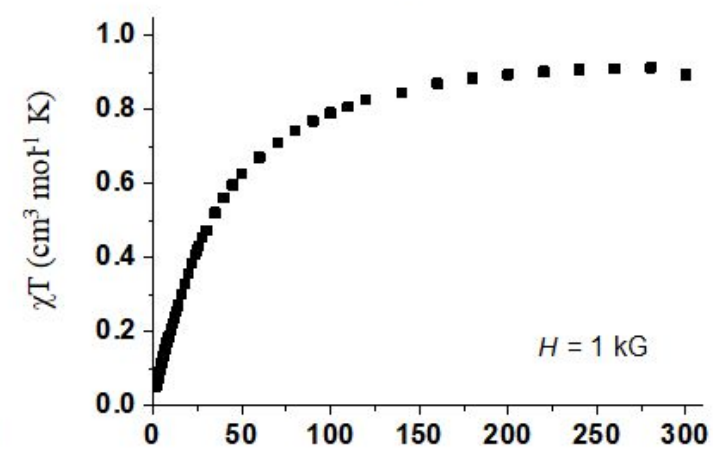

Temperature (K)

Figure S12. Variable-temperature SQUID data of a powdered sample of $\mathbf{1}$
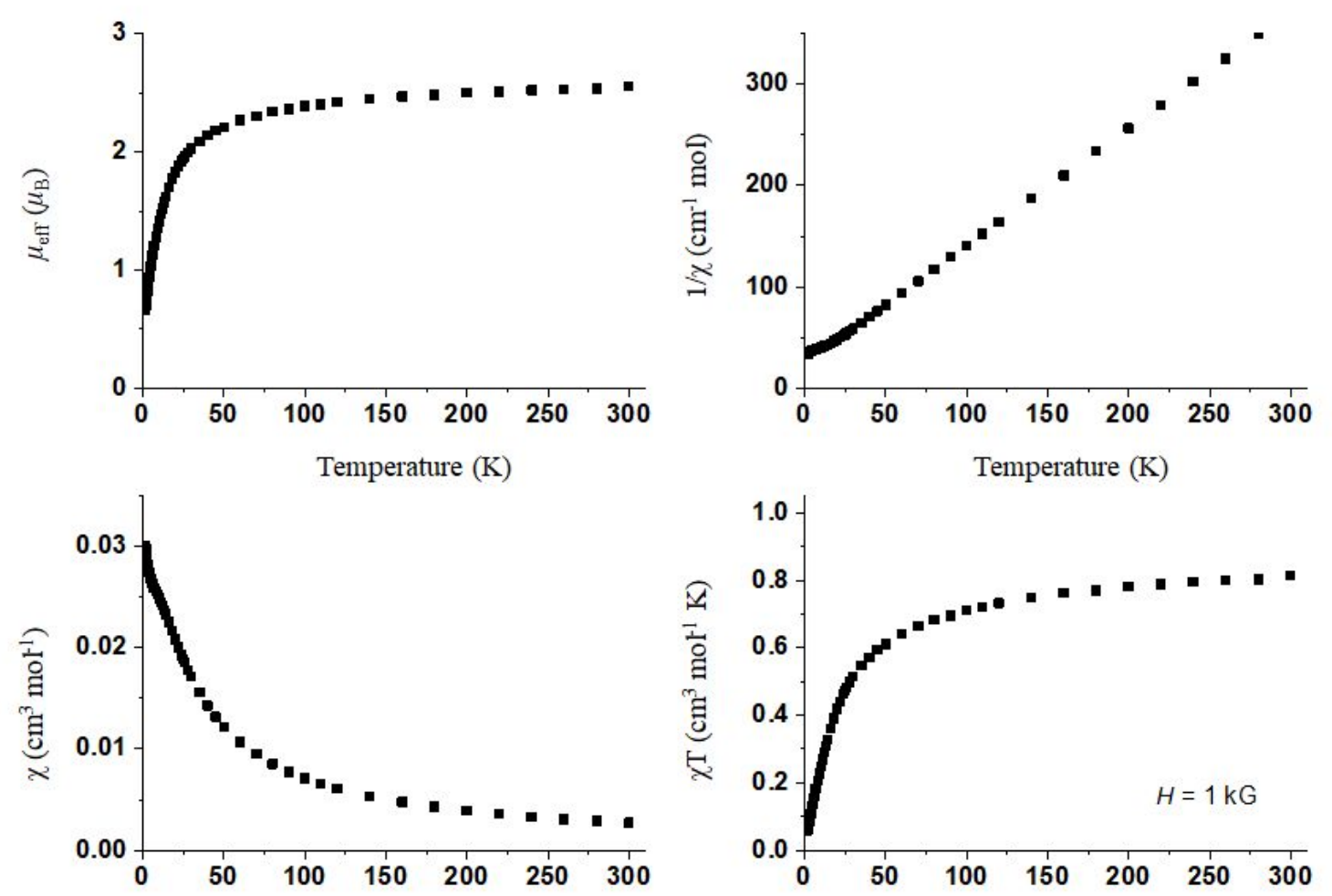

Temperature (K)

Temperature (K)

Figure S13. Variable-temperature SQUID data of a powdered sample of 3. 

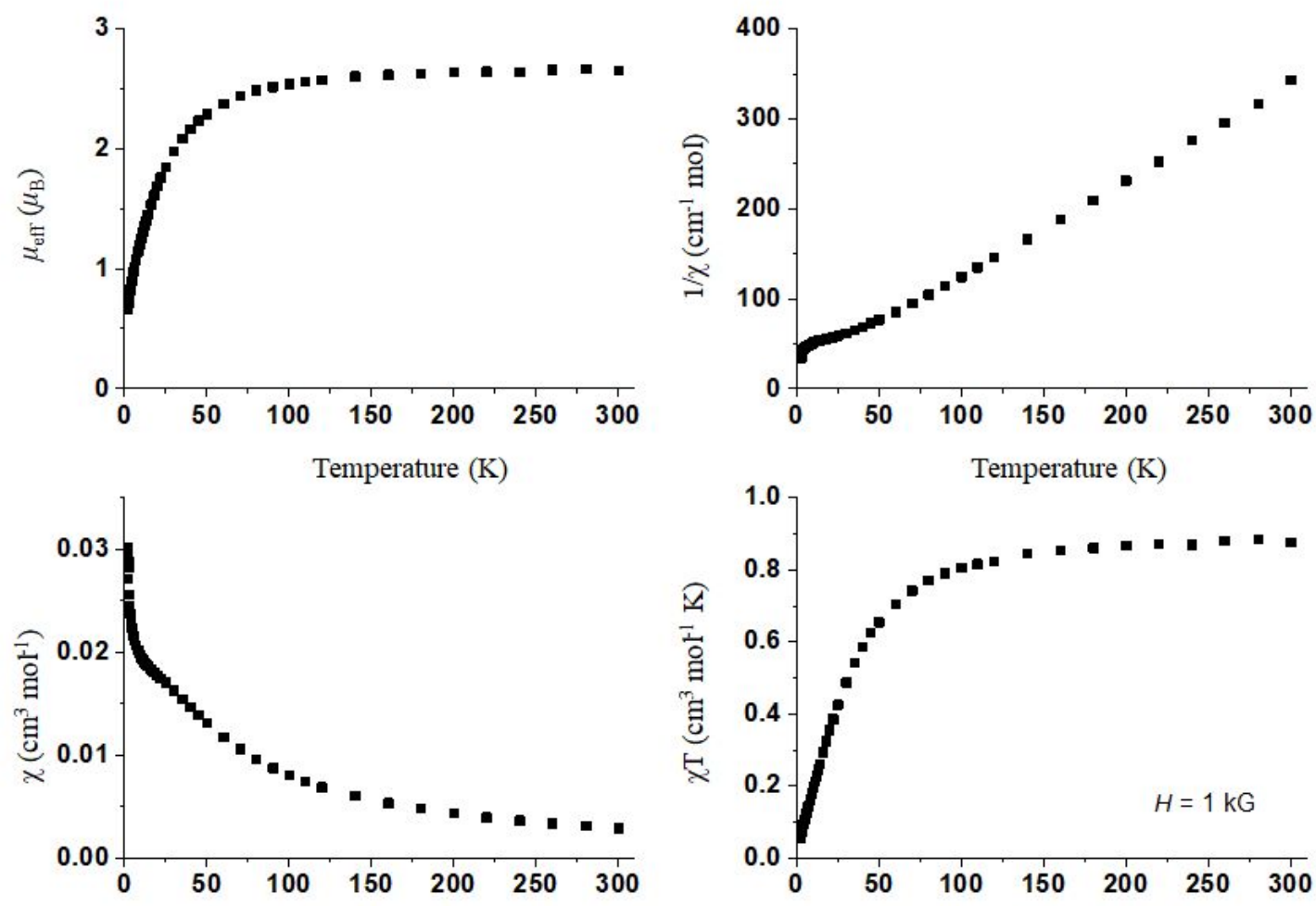

Temperature (K)

Temperature (K)

Figure S14. Variable-temperature SQUID data of a powdered sample of 5.

UV-Vis Spectroscopic Data

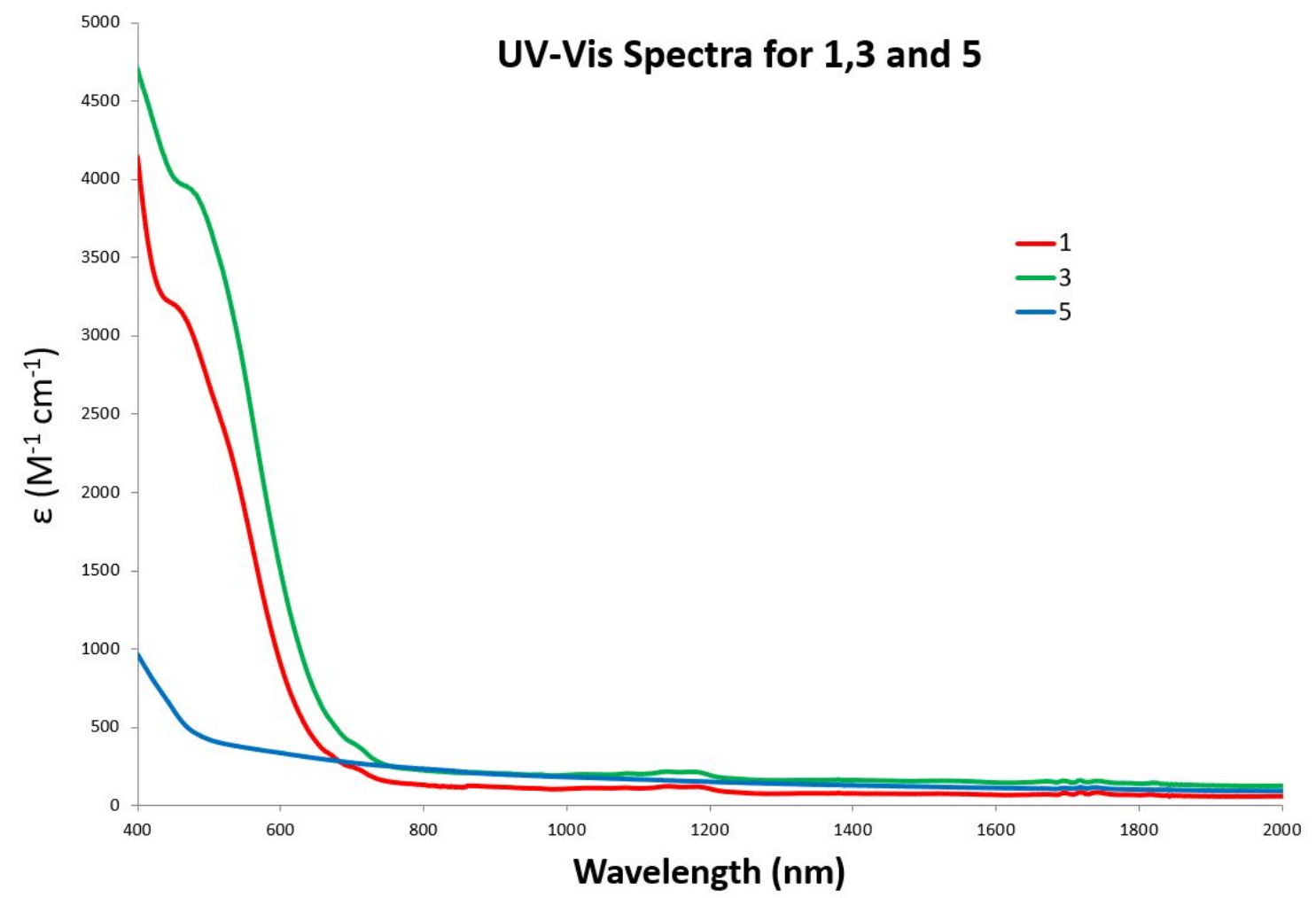

Figure S15. UV-Vis spectra for 1, 3 and $\mathbf{5}$. 


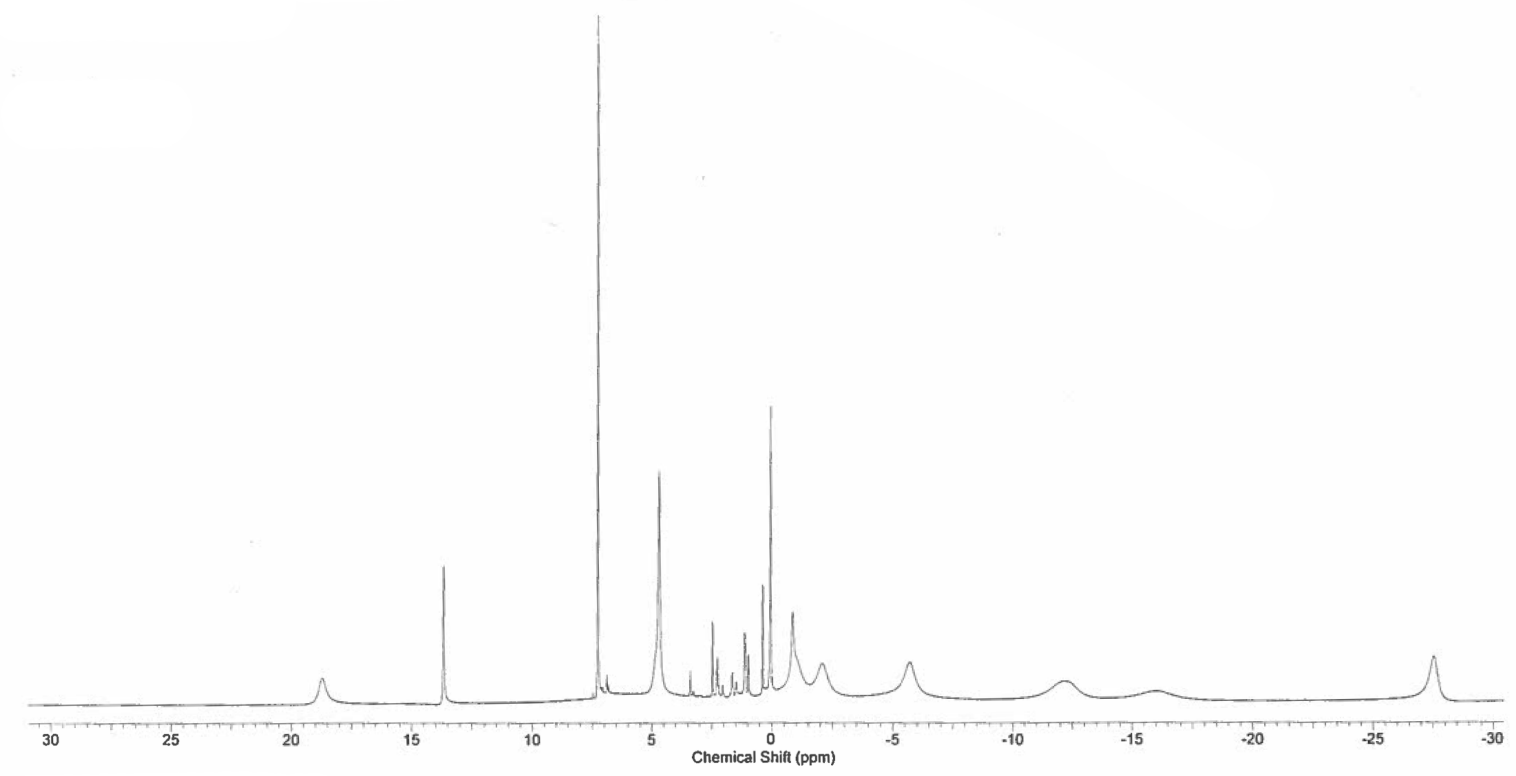

Figure S16. ${ }^{1} \mathrm{H}$ NMR spectrum of 1 in $\mathrm{C}_{6} \mathrm{D}_{6}$.

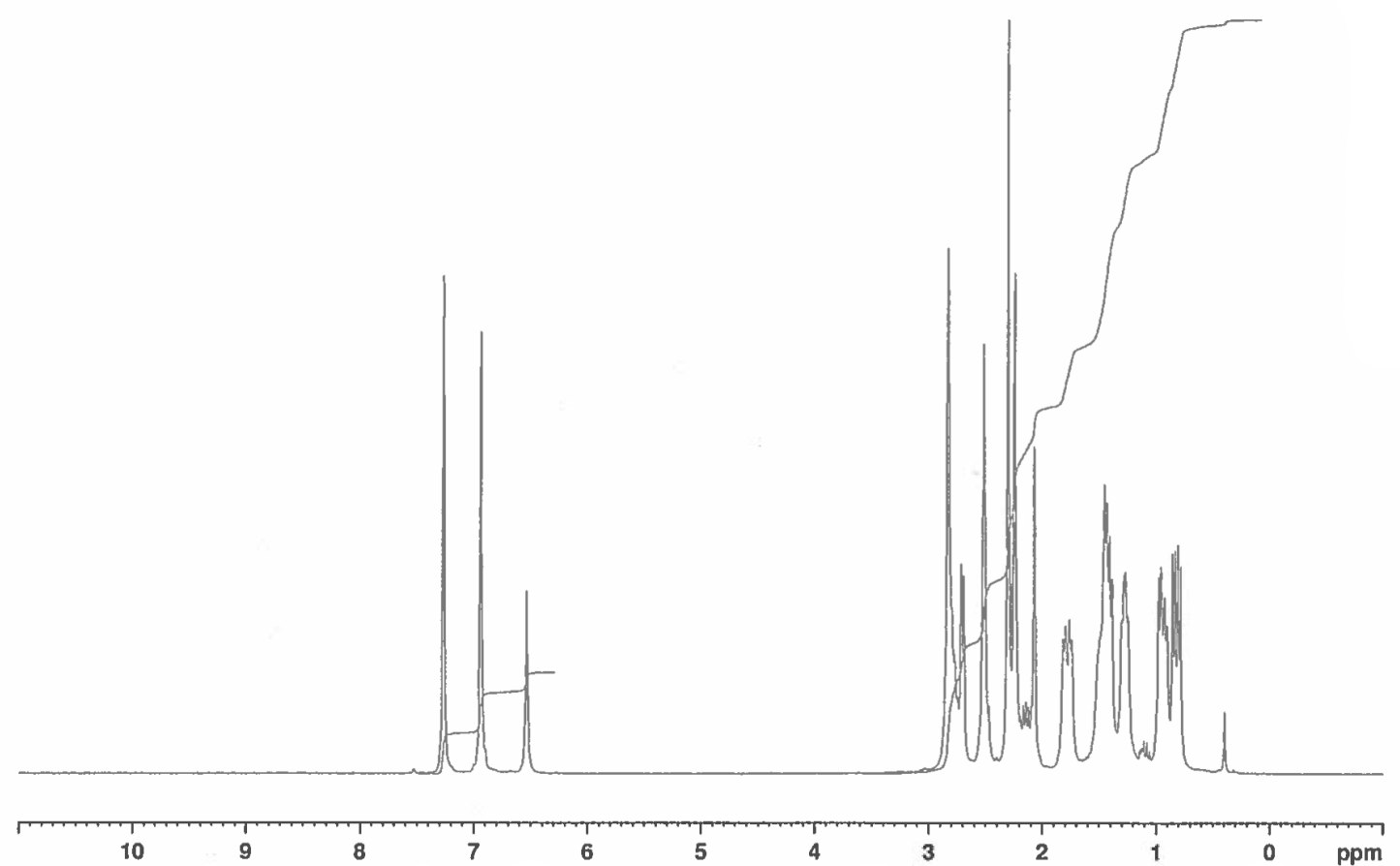

Figure S17. ${ }^{1} \mathrm{H}$ NMR spectrum of 2 in $\mathrm{C}_{6} \mathrm{D}_{6}$. The resonance at $7.27 \mathrm{ppm}$ is residual benzene and should be $7.16 \mathrm{ppm}$, but the assigned resonances are quoted relative to the correct reference value. 


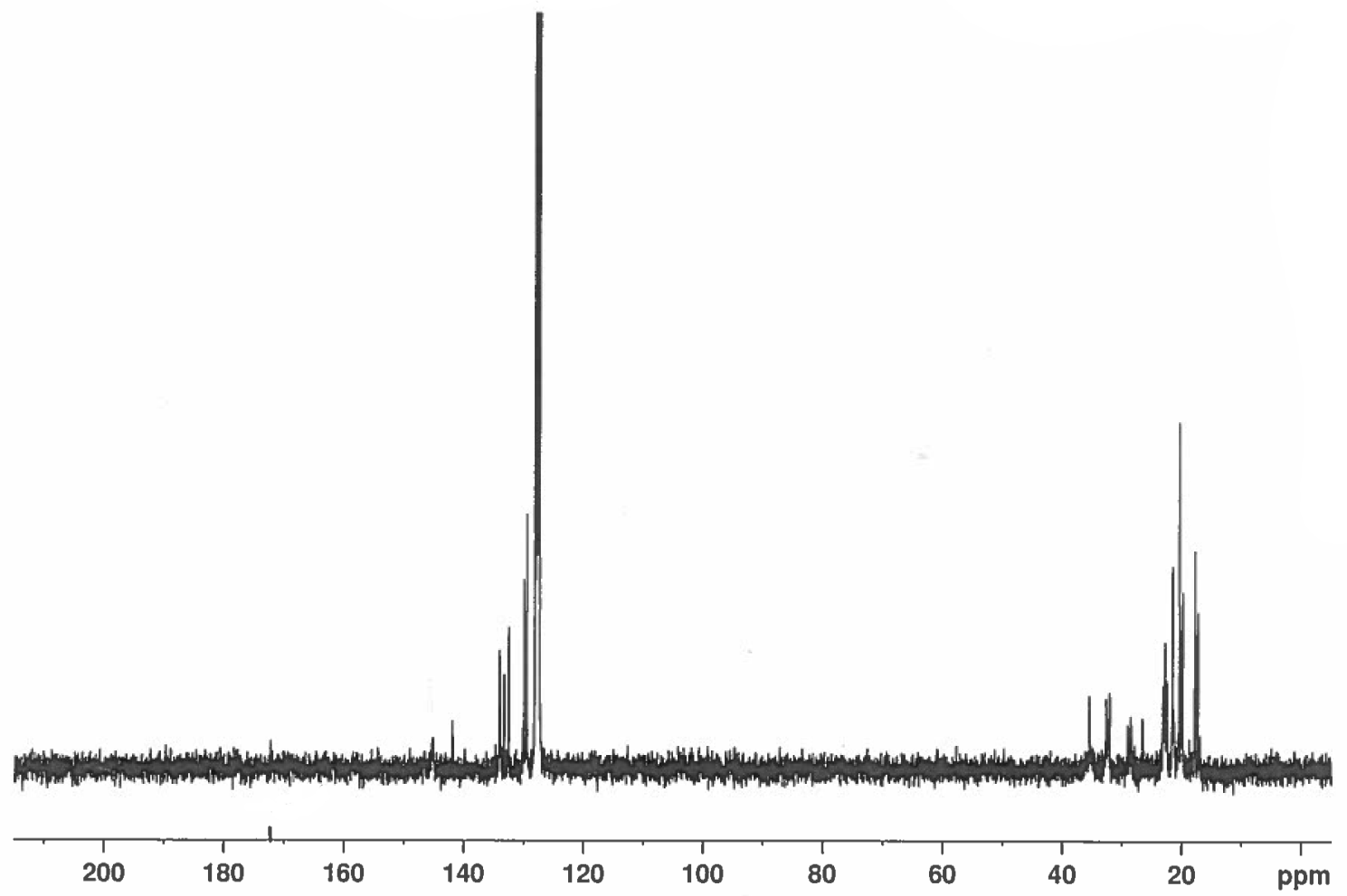

Figure S18. ${ }^{13} \mathrm{C}\left\{{ }^{1} \mathrm{H}\right\}$ NMR spectrum of 2 in $\mathrm{C}_{6} \mathrm{D}_{6}$.

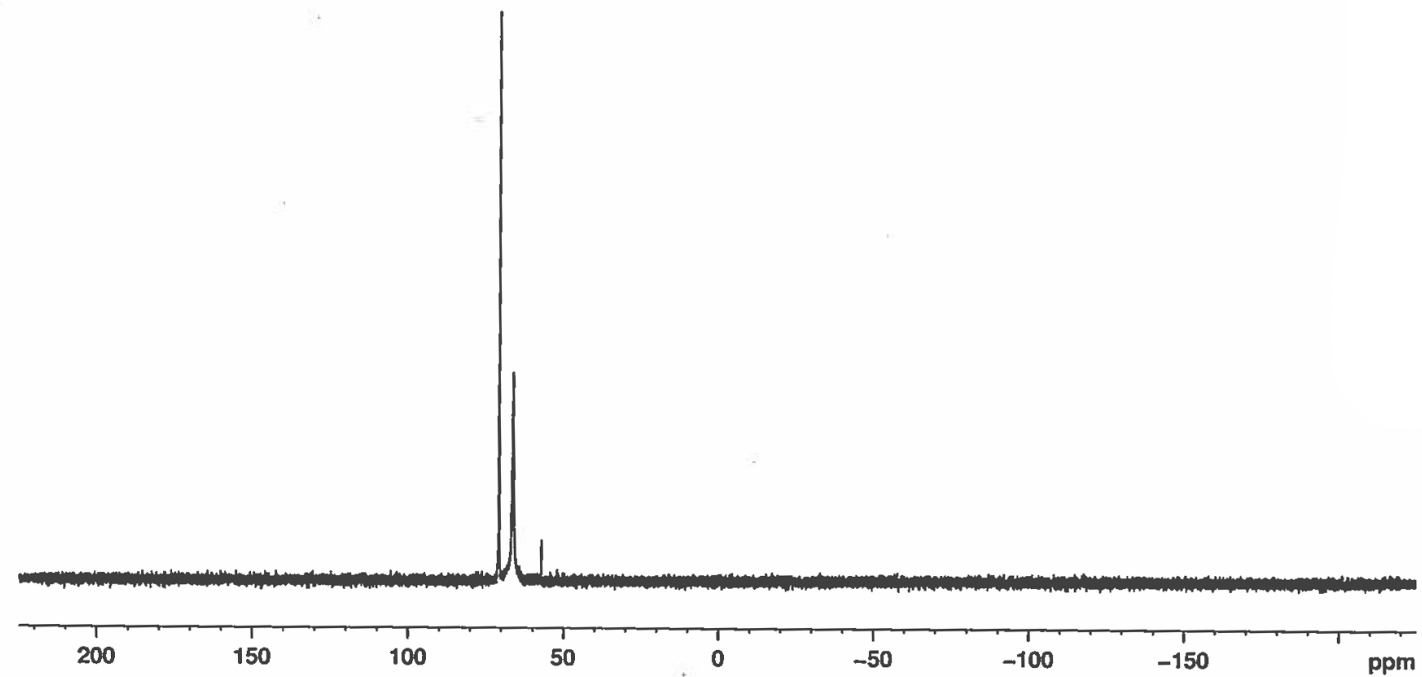

Figure S19. ${ }^{31} \mathrm{P}\left\{{ }^{1} \mathrm{H}\right\}$ NMR spectrum of 2 in $\mathrm{C}_{6} \mathrm{D}_{6}$. 


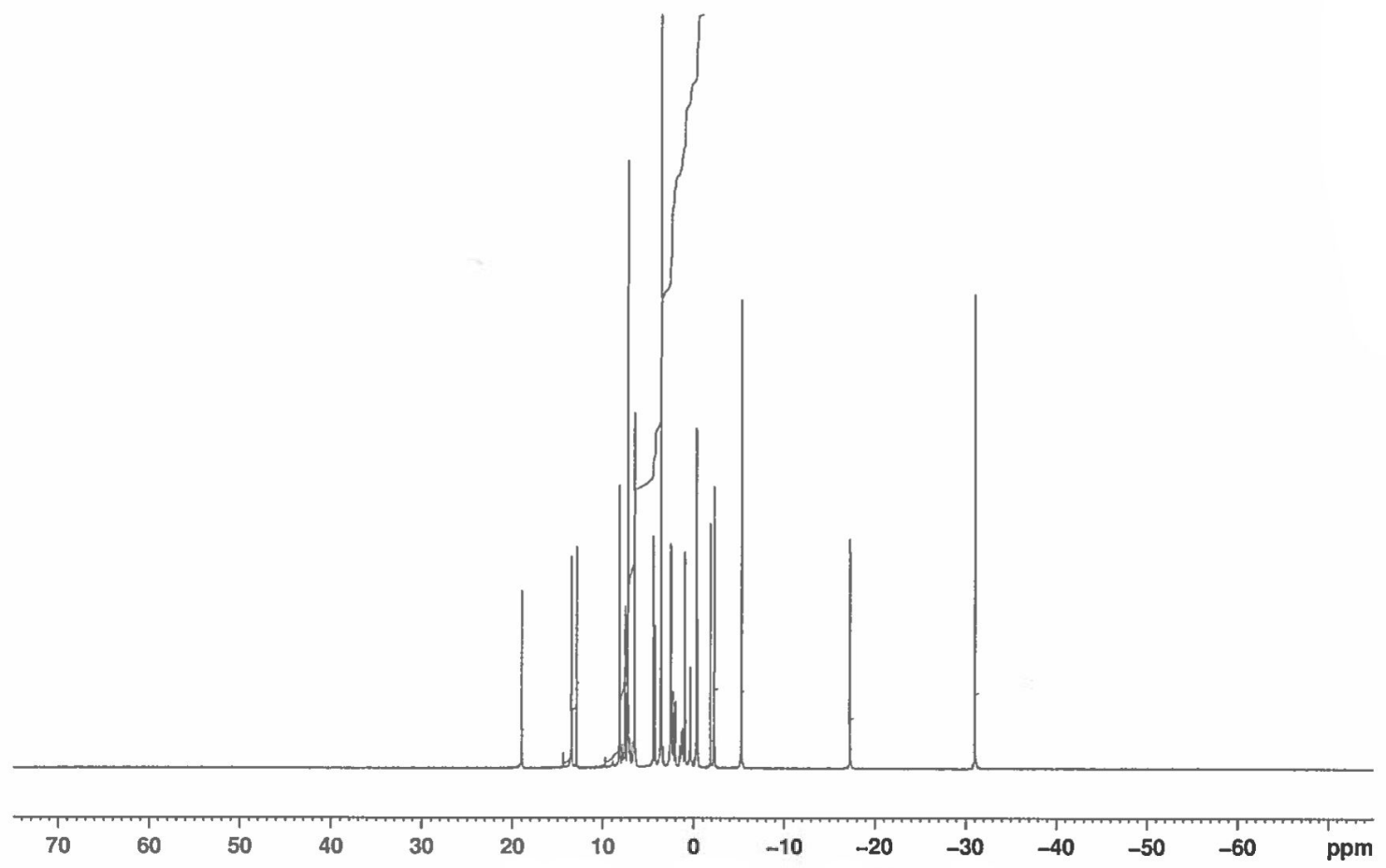

Figure S20. ${ }^{1} \mathrm{H}$ NMR spectrum of 3 in $\mathrm{C}_{6} \mathrm{D}_{6}$. The resonance at $7.27 \mathrm{ppm}$ is residual benzene and should be $7.16 \mathrm{ppm}$, but the assigned resonances are quoted relative to the correct reference value.

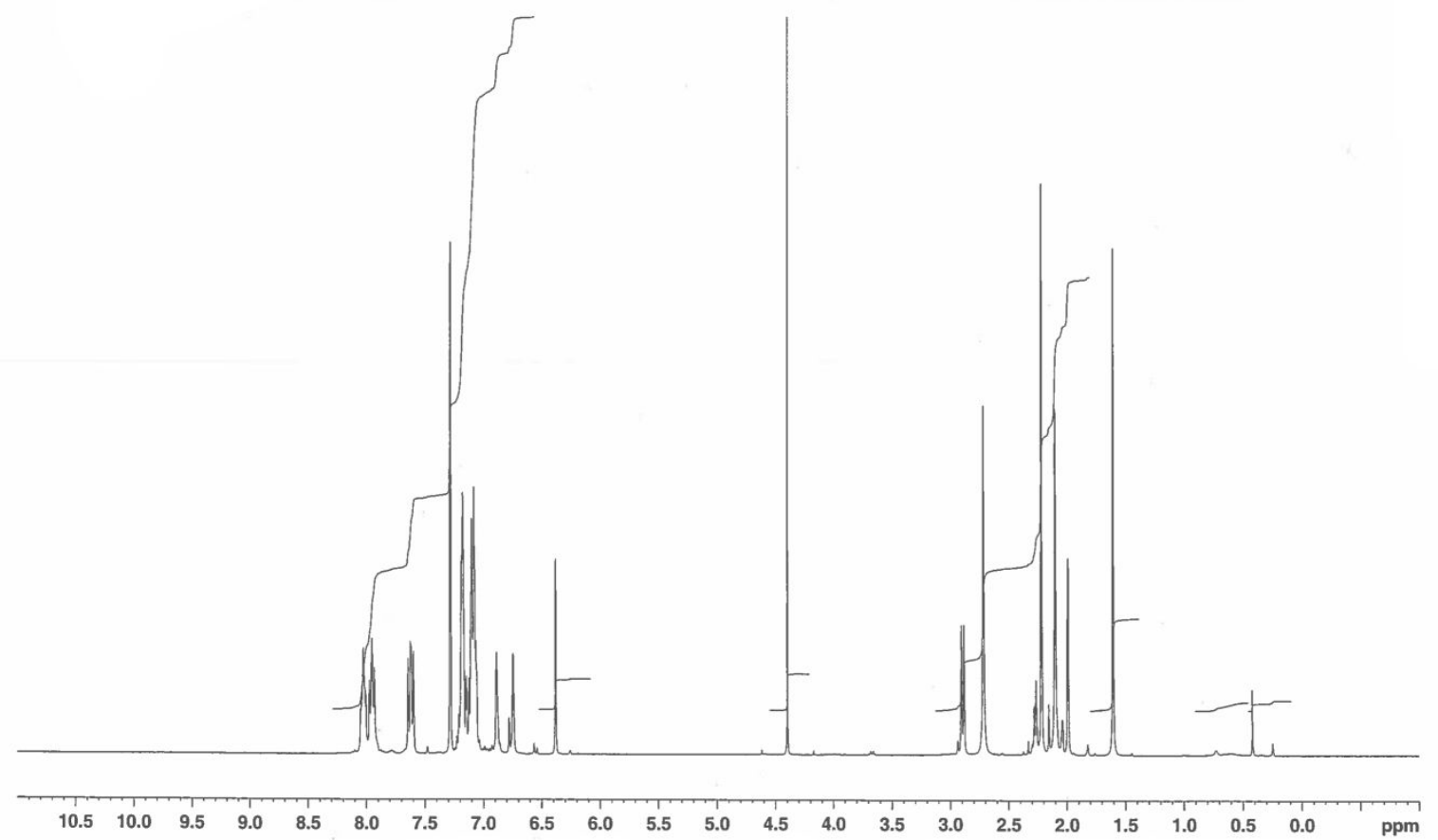

Figure S21. ${ }^{1} \mathrm{H}$ NMR spectrum of 4 in $\mathrm{C}_{6} \mathrm{D}_{6}$. The resonance at $7.28 \mathrm{ppm}$ is residual benzene and should be $7.16 \mathrm{ppm}$, but the assigned resonances are quoted relative to the correct reference value. 


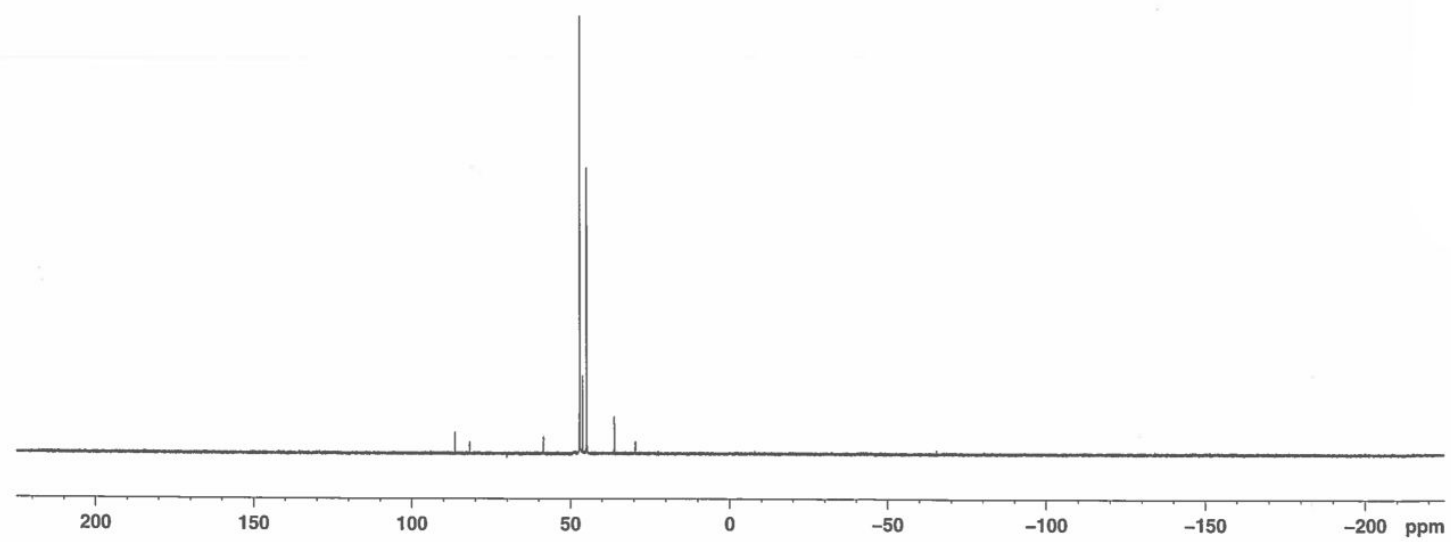

Figure S22. ${ }^{31} \mathrm{P}\left\{{ }^{1} \mathrm{H}\right\}$ NMR spectrum of 4 in $\mathrm{C}_{6} \mathrm{D}_{6}$.

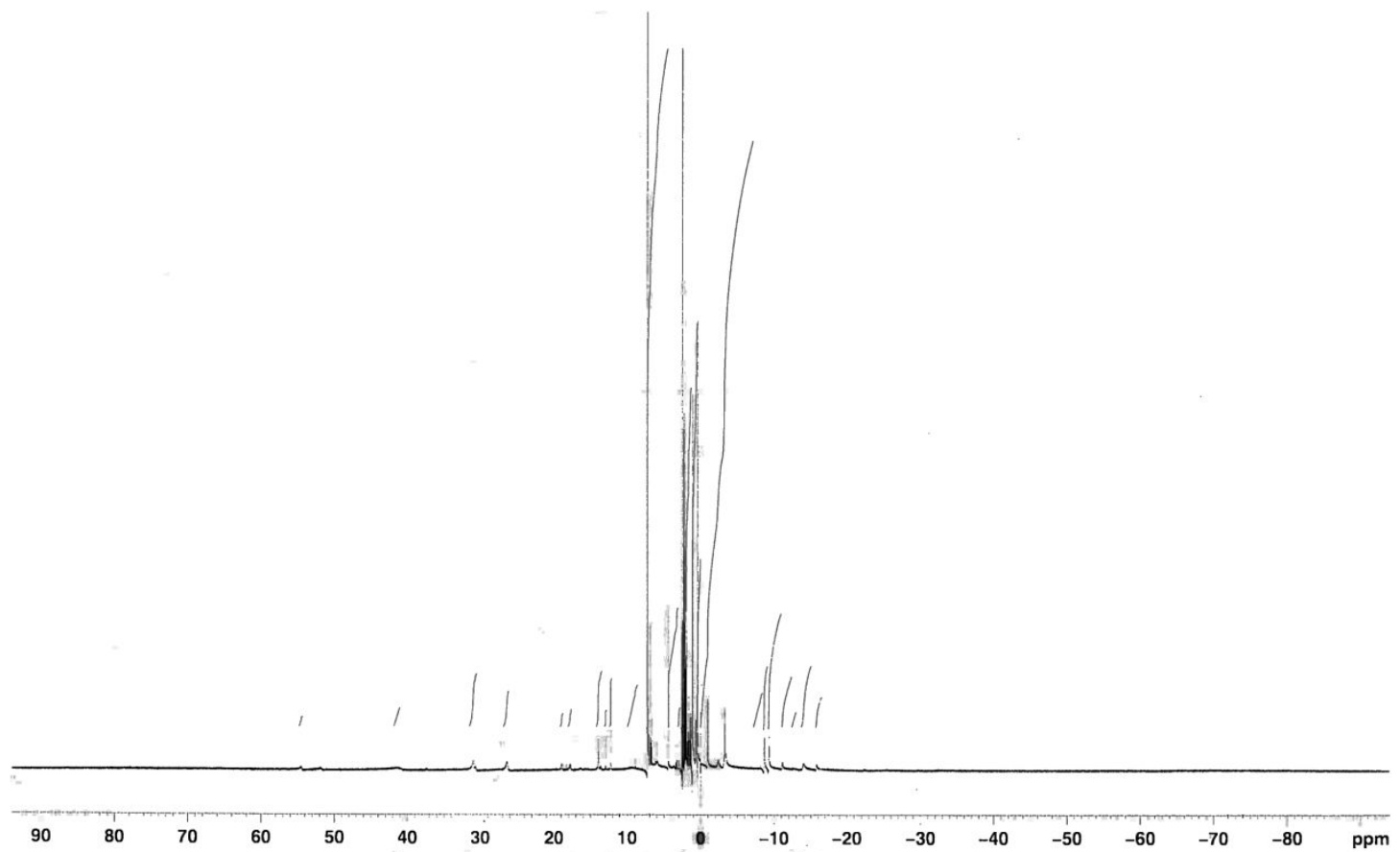

Figure S23. ${ }^{1} \mathrm{H}$ NMR spectrum of 5 in $\mathrm{C}_{6} \mathrm{D}_{6}$. 


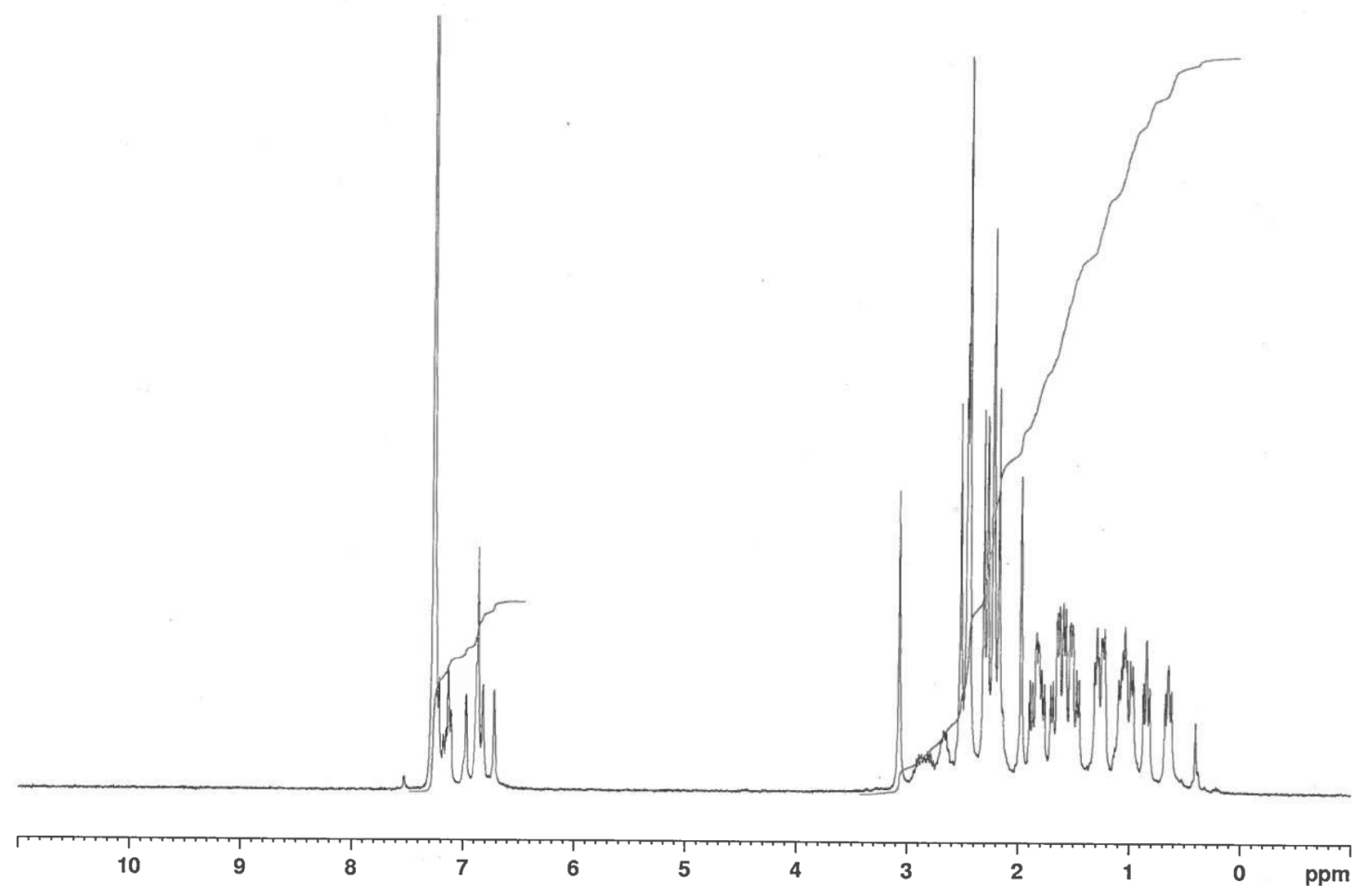

Figure S24. ${ }^{1} \mathrm{H}$ NMR spectrum of 6 in $\mathrm{C}_{6} \mathrm{D}_{6}$. The resonance at $7.27 \mathrm{ppm}$ is residual benzene and should be $7.16 \mathrm{ppm}$, but the assigned resonances are quoted relative to the correct reference value.

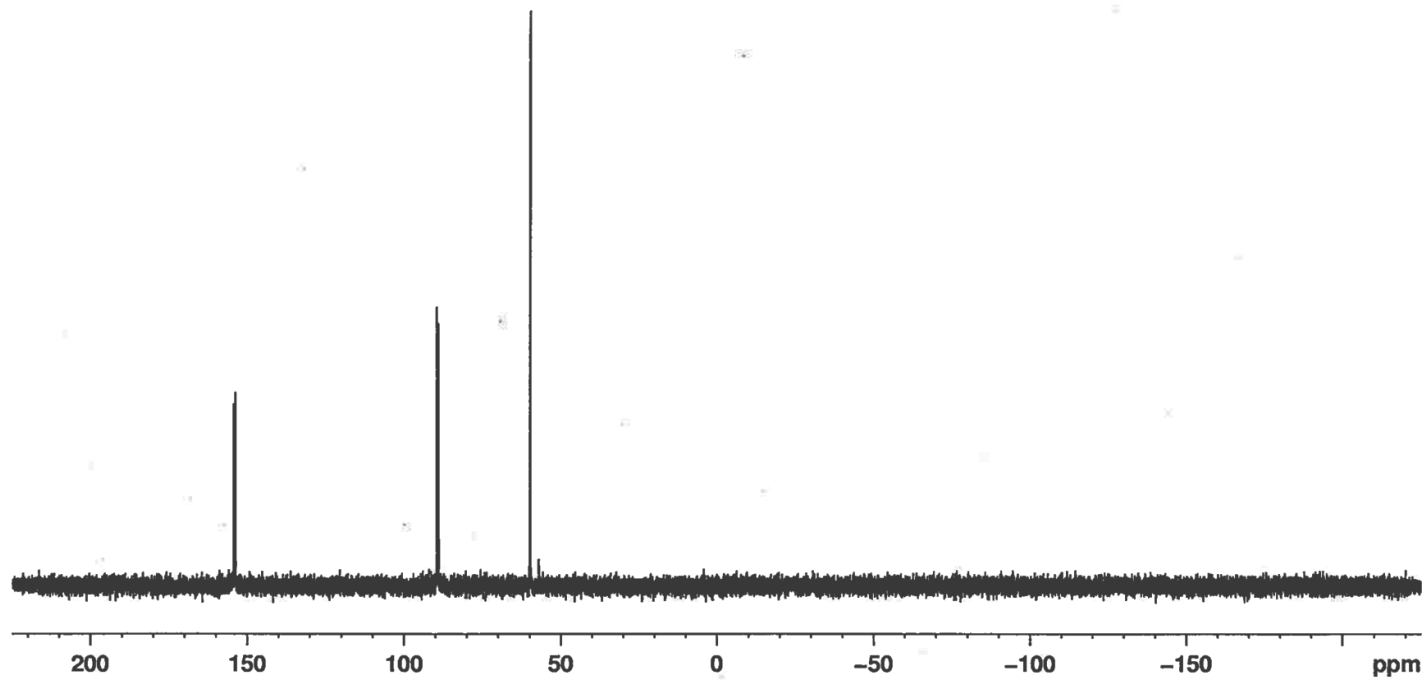

Figure S25. ${ }^{31} \mathrm{P}\left\{{ }^{1} \mathrm{H}\right\}$ NMR spectrum of 6 in $\mathrm{C}_{6} \mathrm{D}_{6}$. 


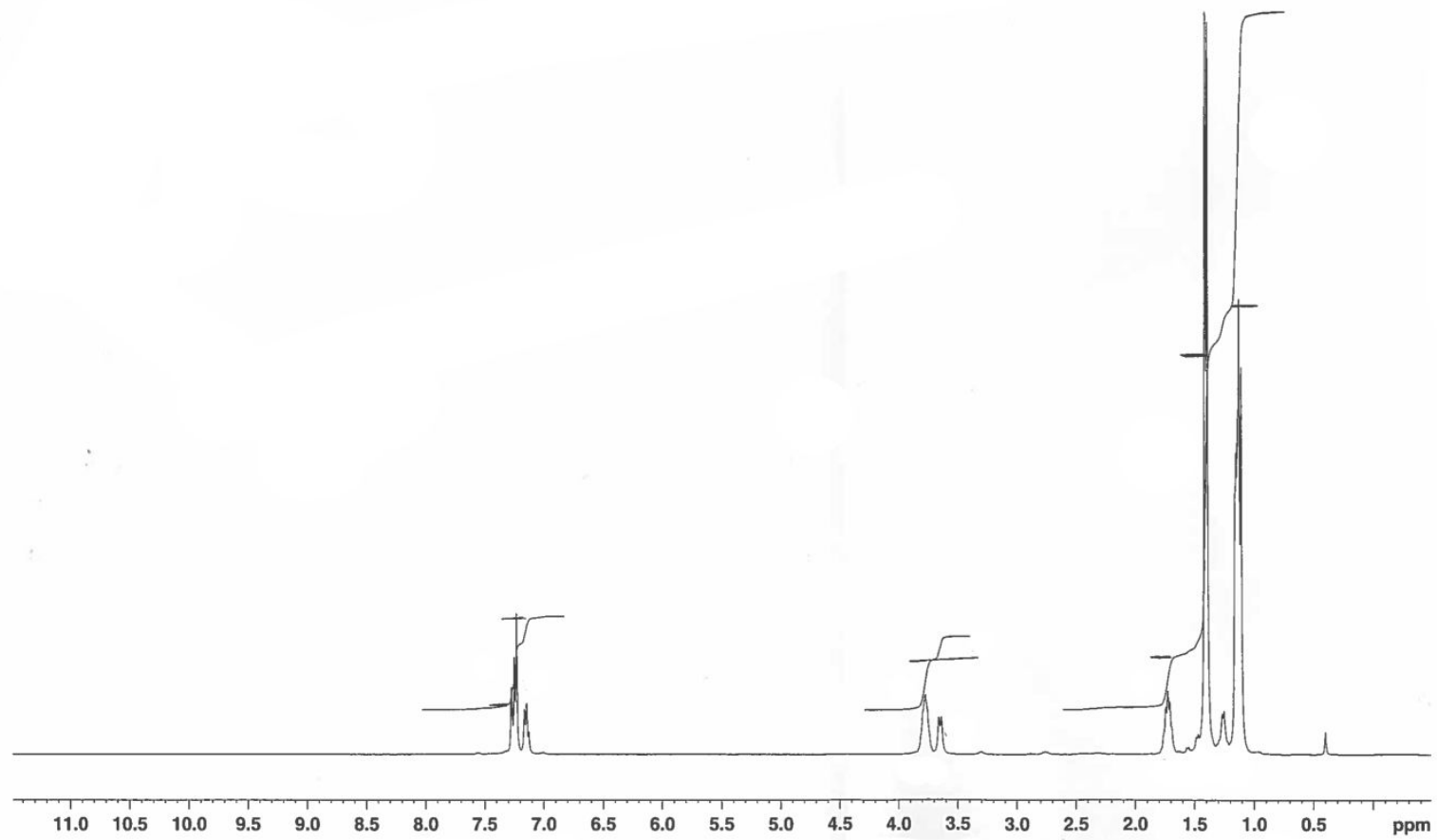

Figure S26. ${ }^{1} \mathrm{H}$ NMR spectrum of $\operatorname{DippN}(\mathrm{H}) \mathrm{P}^{\mathrm{i}} \mathrm{Pr}_{2}$ in $\mathrm{C}_{6} \mathrm{D}_{6}$.

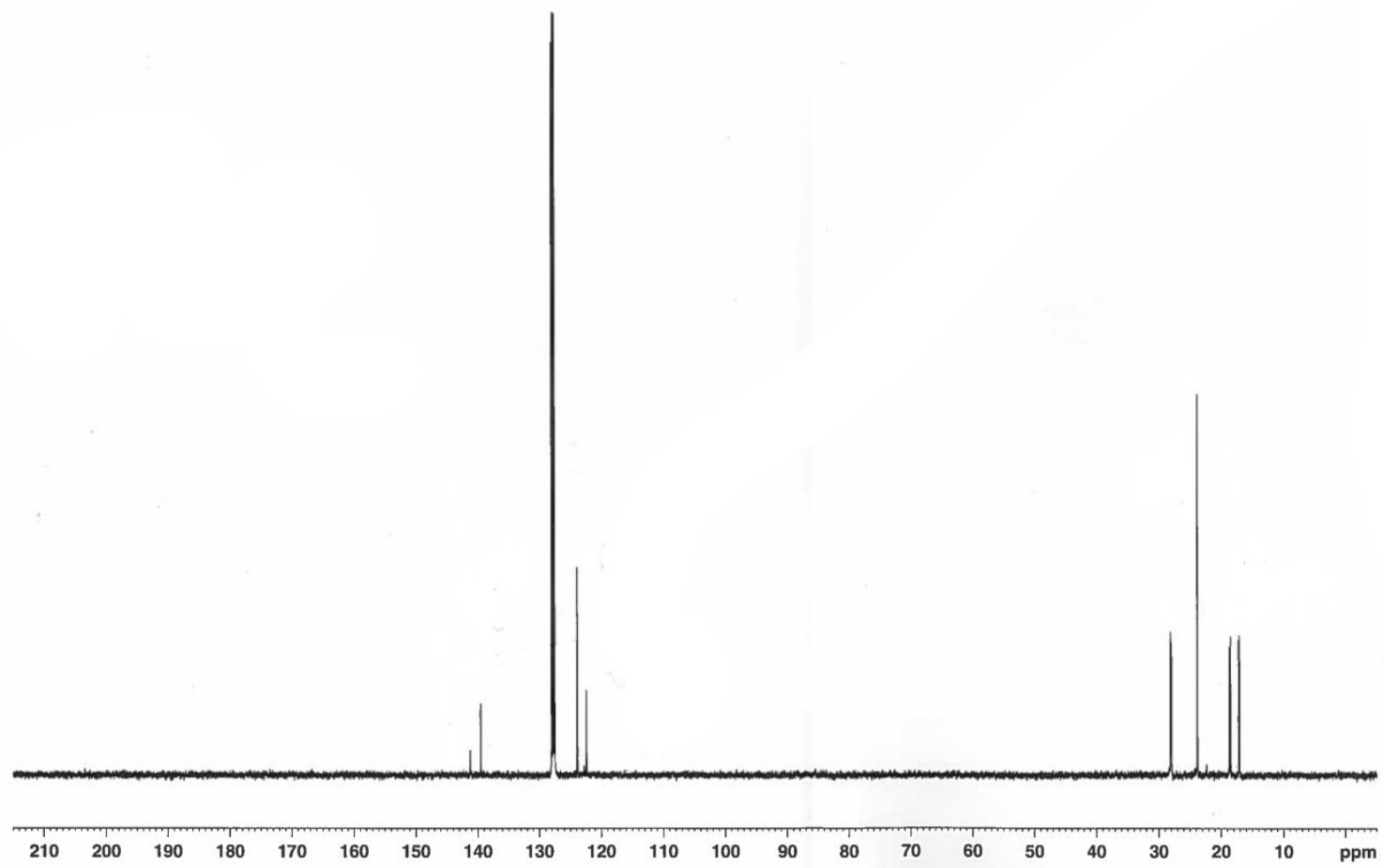

Figure S27. ${ }^{13} \mathrm{C}\left\{{ }^{1} \mathrm{H}\right\}$ NMR spectrum of DippN(H)PiPr ${ }_{2}$ in $\mathrm{C}_{6} \mathrm{D}_{6}$. 


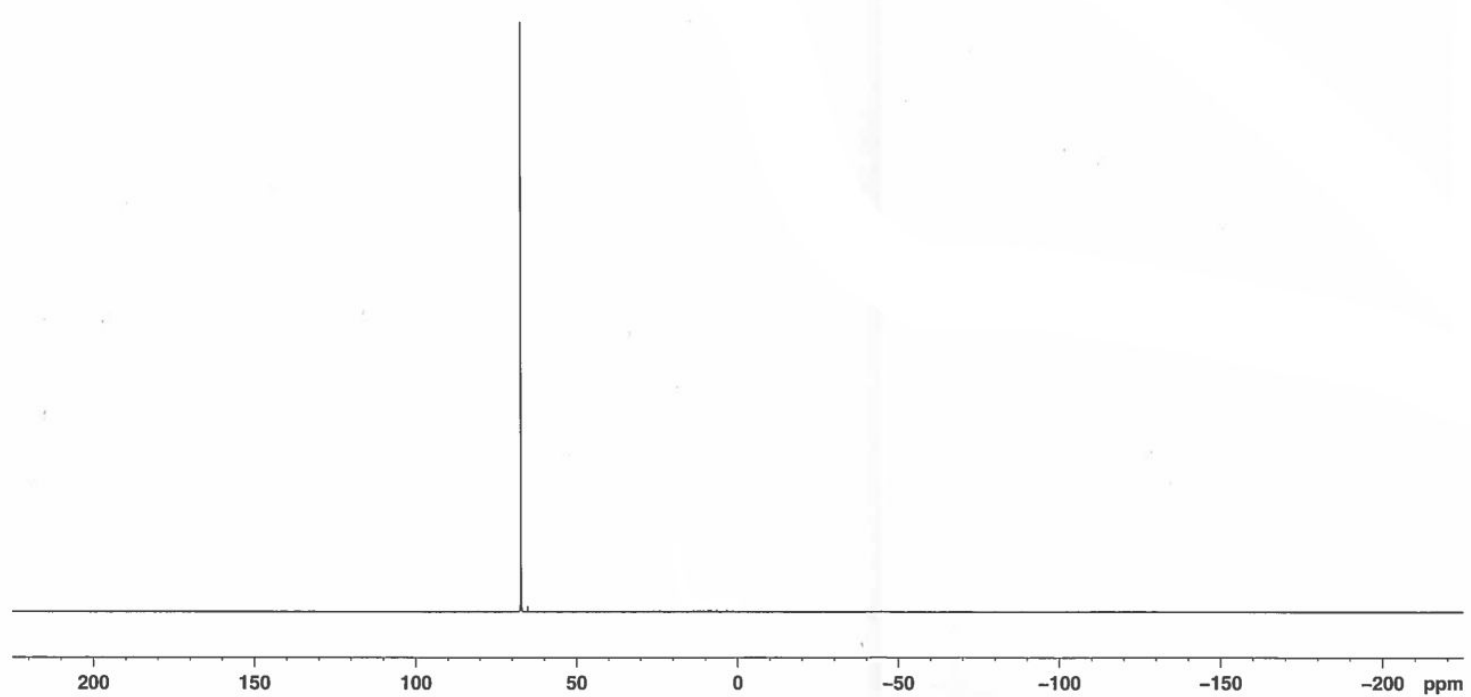

Figure S28. ${ }^{31} \mathrm{P}\left\{{ }^{1} \mathrm{H}\right\}$ NMR spectrum of $\operatorname{DippN}(\mathrm{H}) \mathrm{P}^{\mathrm{i}} \mathrm{Pr}_{2}$ in $\mathrm{C}_{6} \mathrm{D}_{6}$.

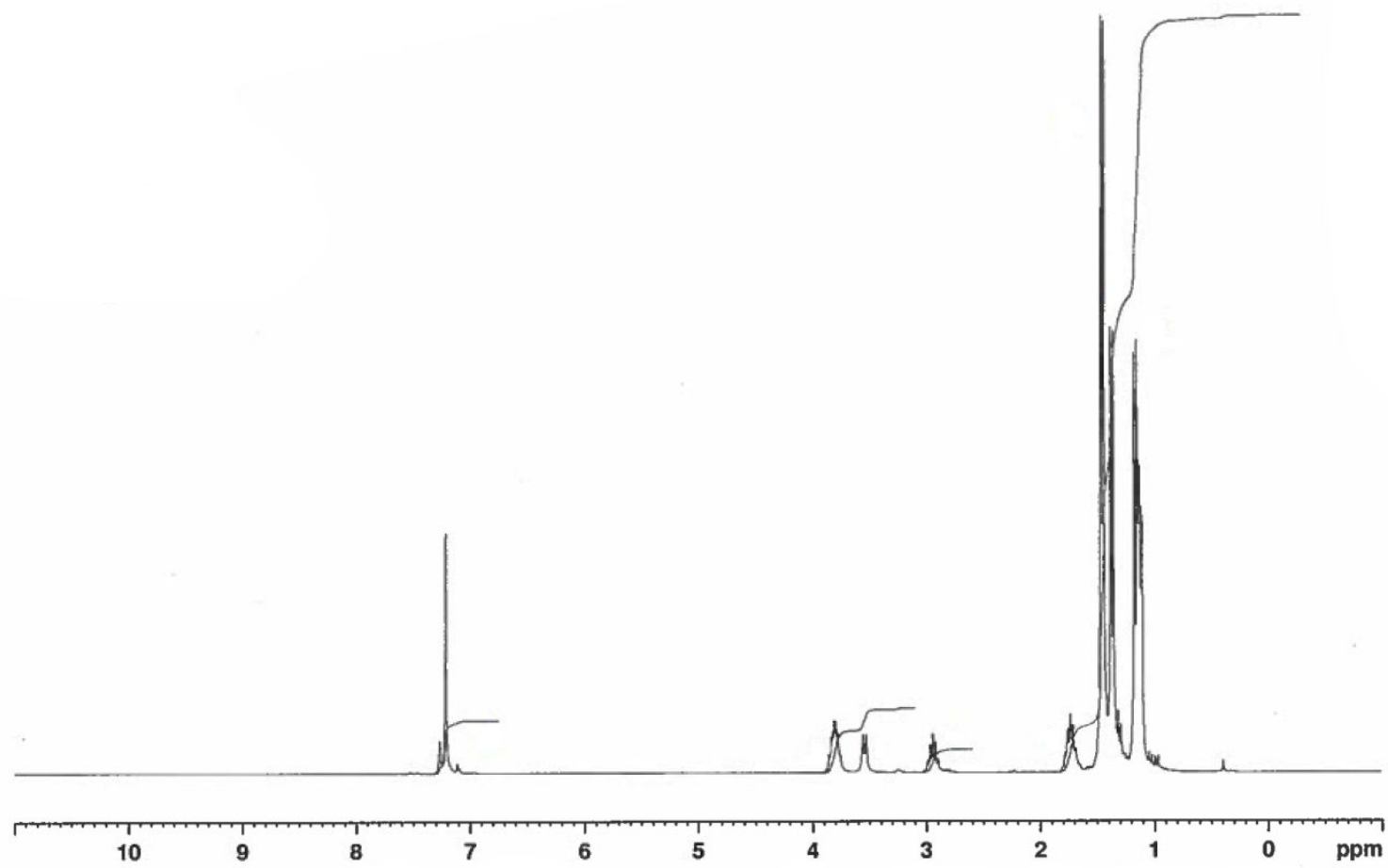

Figure S29. ${ }^{1} \mathrm{H}$ NMR spectrum of $\operatorname{TrippN}(\mathrm{H}) \mathrm{P}^{\mathrm{i}} \mathrm{Pr}_{2}$ in $\mathrm{C}_{6} \mathrm{D}_{6}$. The resonance at $7.27 \mathrm{ppm}$ is residual benzene and should be $7.16 \mathrm{ppm}$, but the assigned resonances are quoted relative to the correct reference value. 


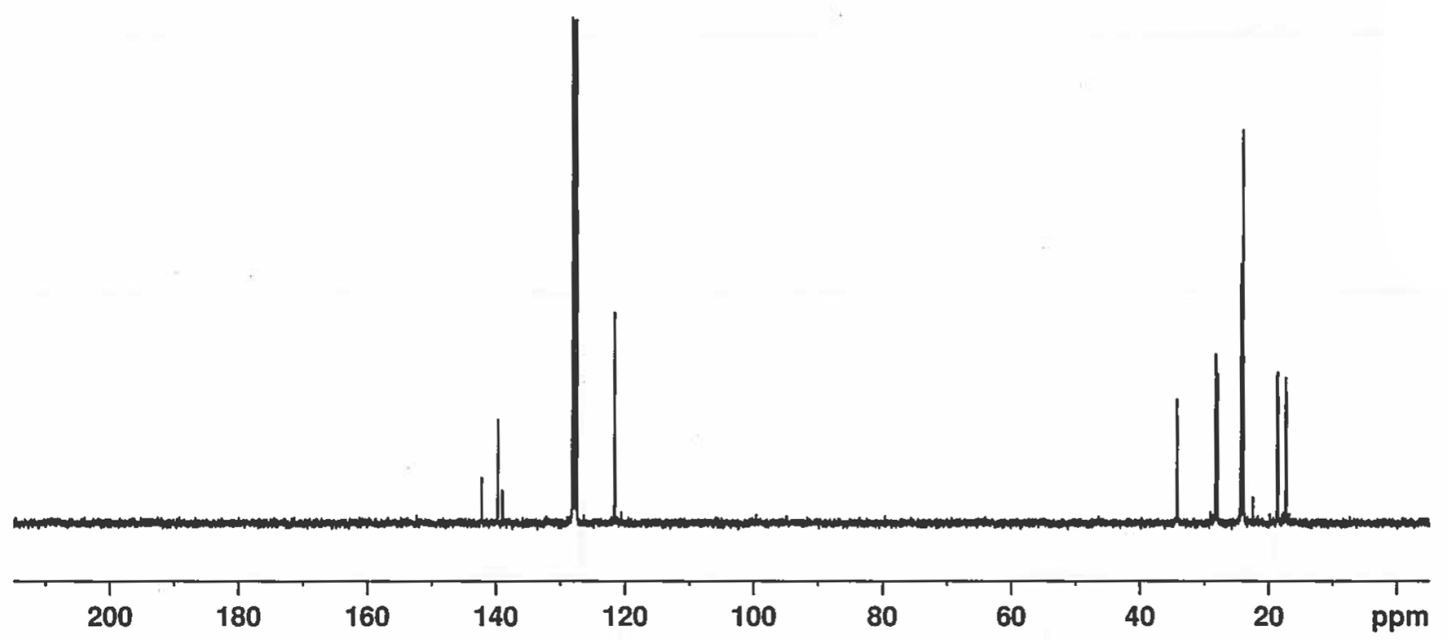

Figure S30. ${ }^{13} \mathrm{C}\left\{{ }^{1} \mathrm{H}\right\}$ NMR spectrum of TrippN(H)PiPr ${ }_{2}$ in $\mathrm{C}_{6} \mathrm{D}_{6}$.

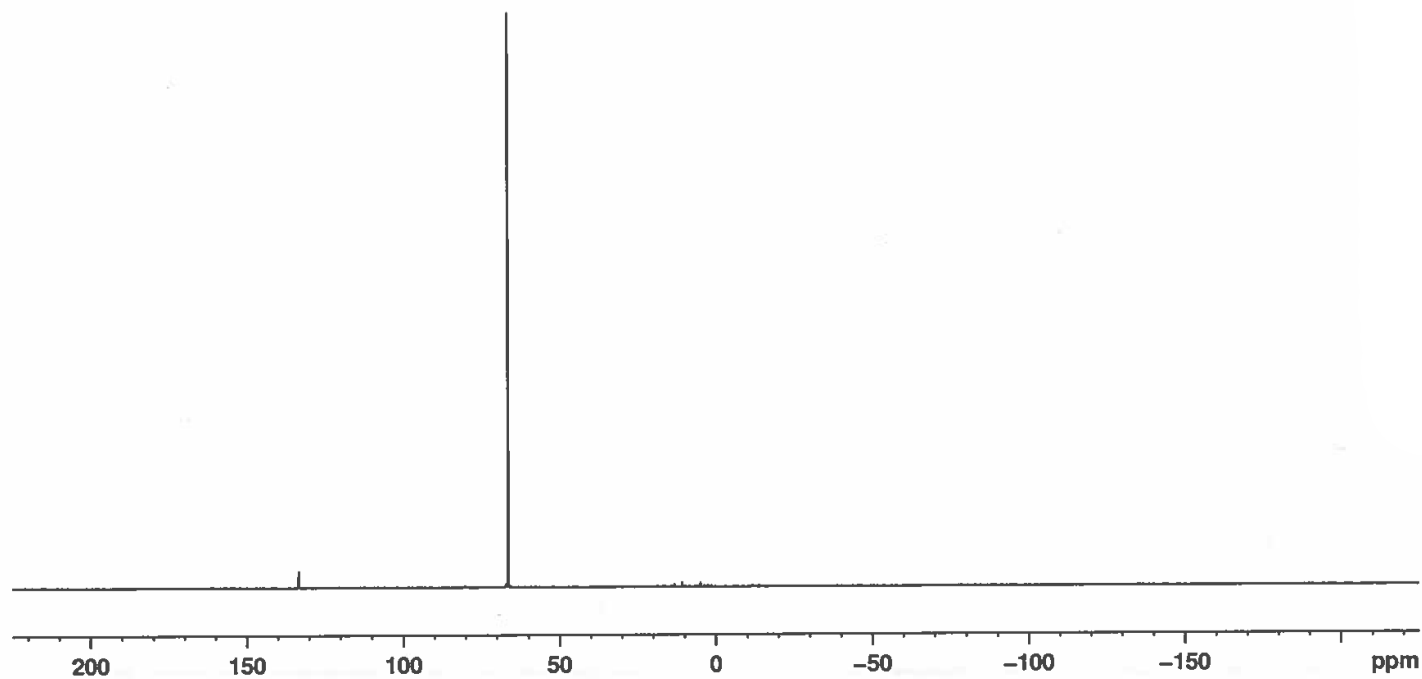

Figure S31. ${ }^{31} \mathrm{P}\left\{{ }^{1} \mathrm{H}\right\}$ NMR spectrum of TrippN(H)PiPr ${ }_{2}$ in $\mathrm{C}_{6} \mathrm{D}_{6}$. 


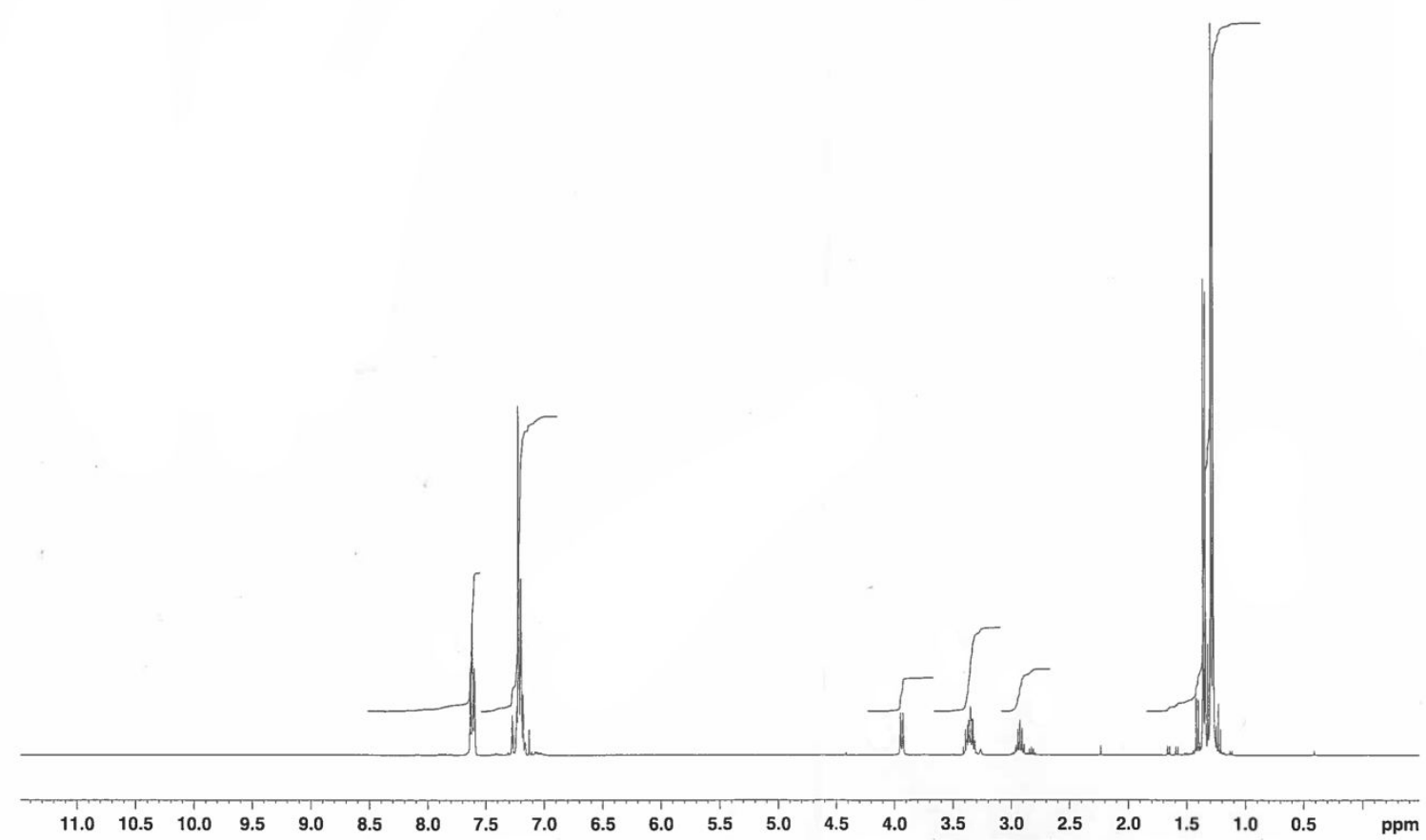

Figure S32. ${ }^{1} \mathrm{H}$ NMR spectrum of TrippN(H)PPh${ }_{2}$ in $\mathrm{C}_{6} \mathrm{D}_{6}$. The resonance at $7.27 \mathrm{ppm}$ is residual benzene and should be $7.16 \mathrm{ppm}$, but the assigned resonances are quoted relative to the correct reference value.

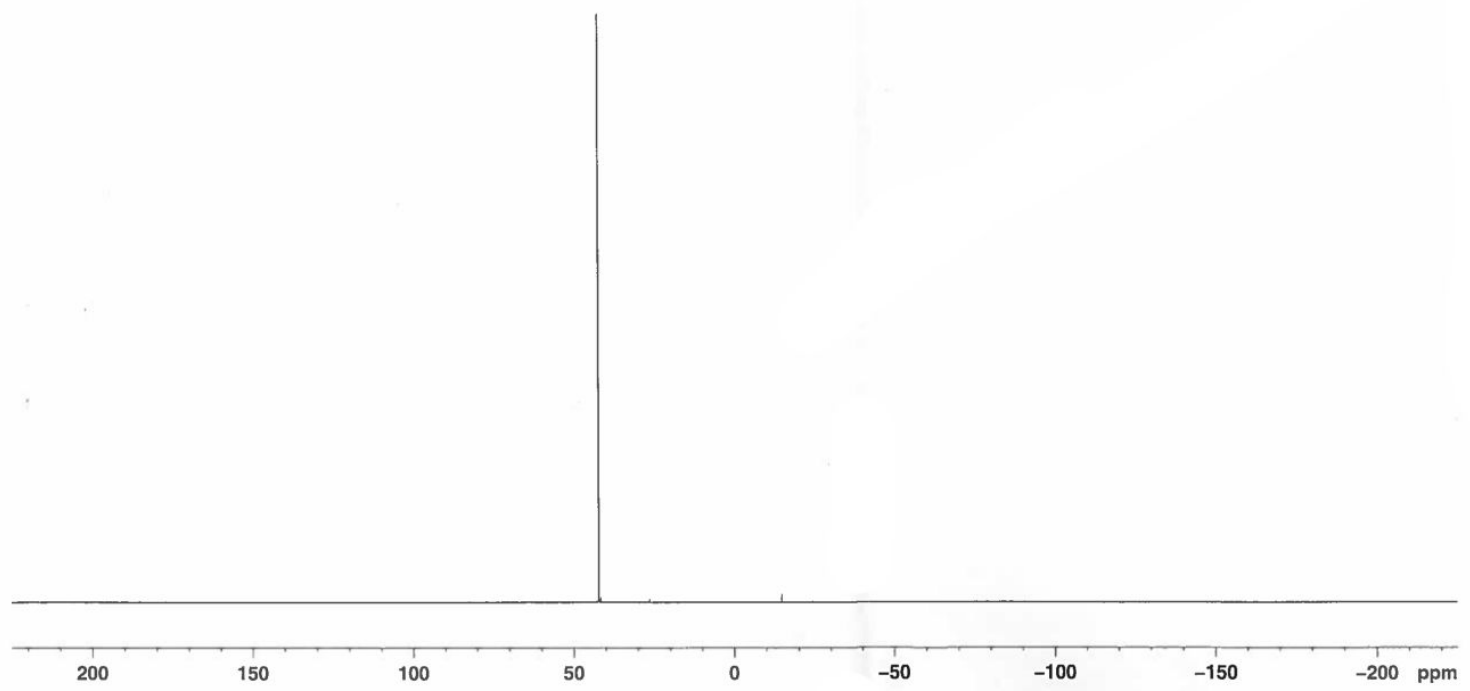

Figure S33. ${ }^{31} \mathrm{P}\left\{{ }^{1} \mathrm{H}\right\}$ NMR spectrum of $\operatorname{TrippN}(\mathrm{H}) \mathrm{PPh}_{2}$ in $\mathrm{C}_{6} \mathrm{D}_{6}$. 


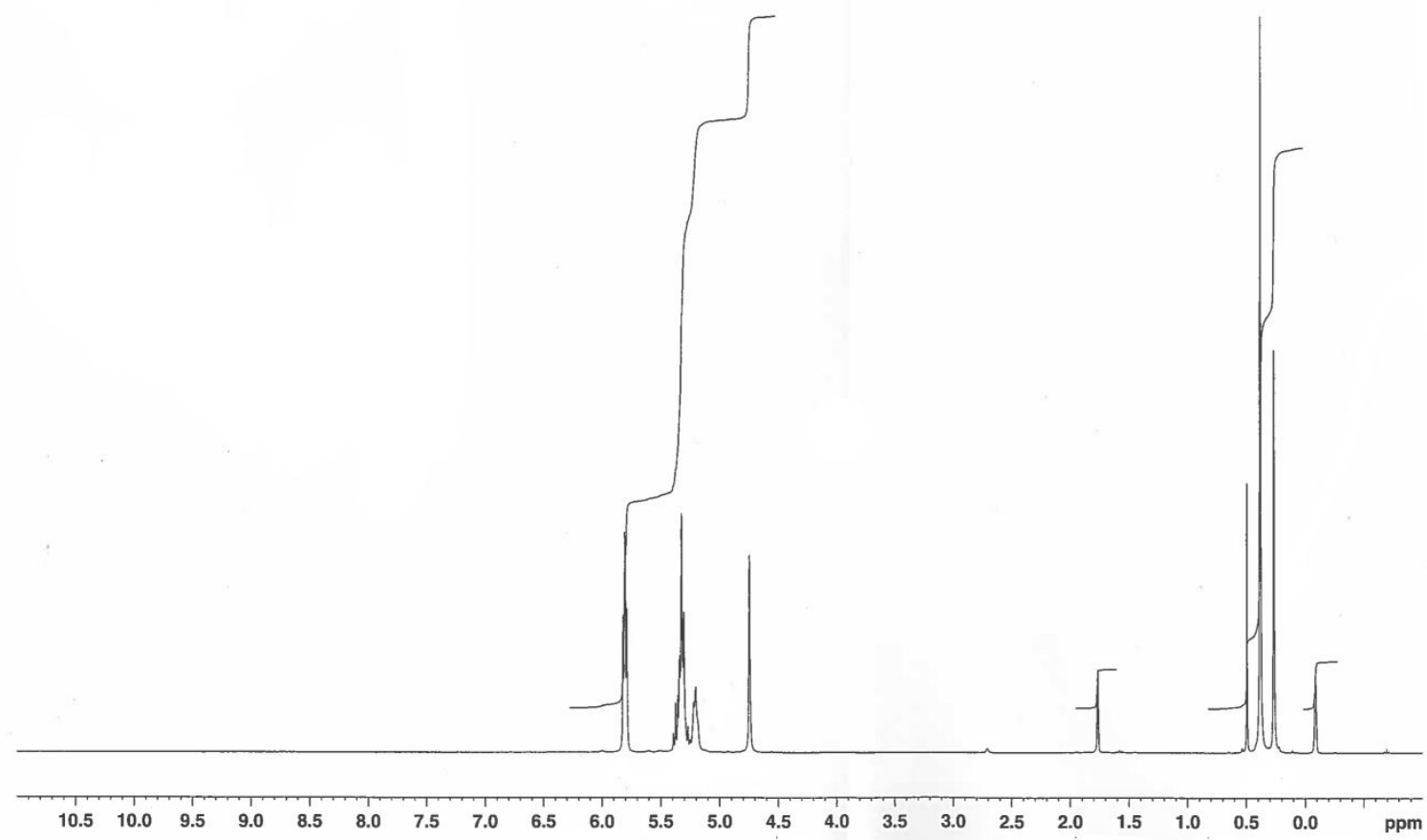

Figure S34. ${ }^{1} \mathrm{H}$ NMR spectrum of $\mathbf{S 1}$ in $\mathrm{D}_{8}$-THF. The printed resonances are relative to the incorrect residual THF resonance. The quoted values are referenced correctly.

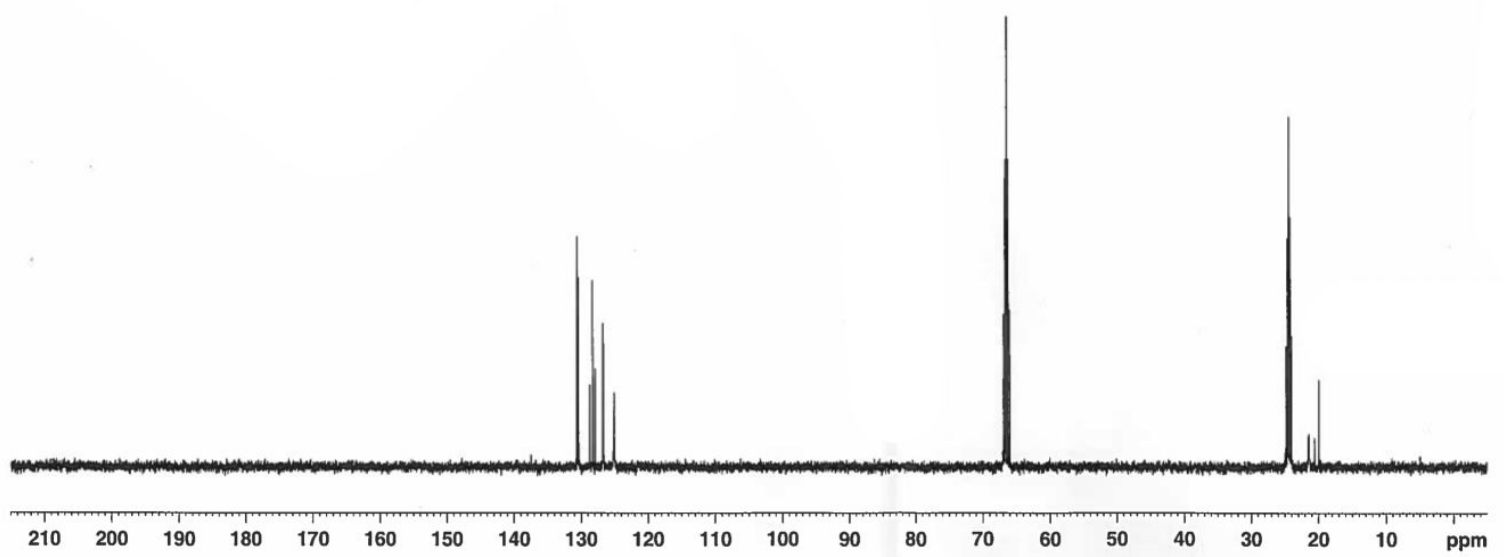

Figure $\mathbf{S 3 5} .{ }^{13} \mathrm{C}\left\{{ }^{1} \mathrm{H}\right\}$ NMR spectrum of $\mathbf{S 1}$ in $\mathrm{D}_{8}$-THF. 


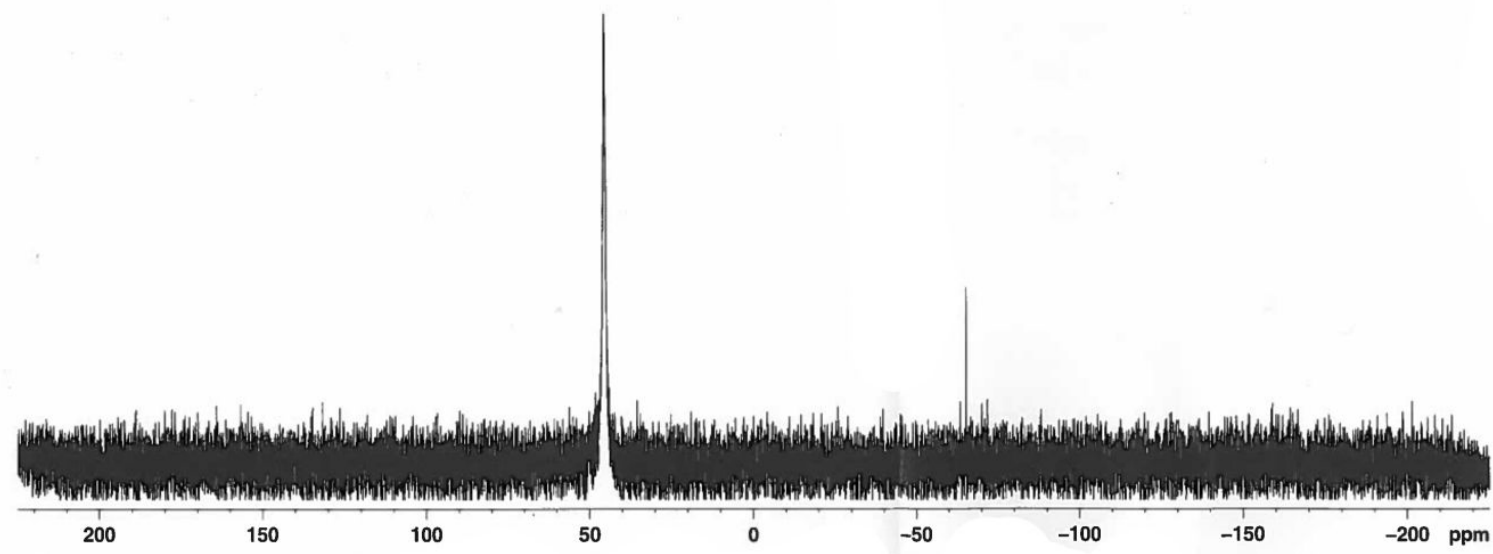

Figure S36. ${ }^{31} \mathrm{P}\left\{{ }^{1} \mathrm{H}\right\}$ NMR spectrum of $\mathbf{S} 1$ in $\mathrm{D}_{8}-\mathrm{THF}$.

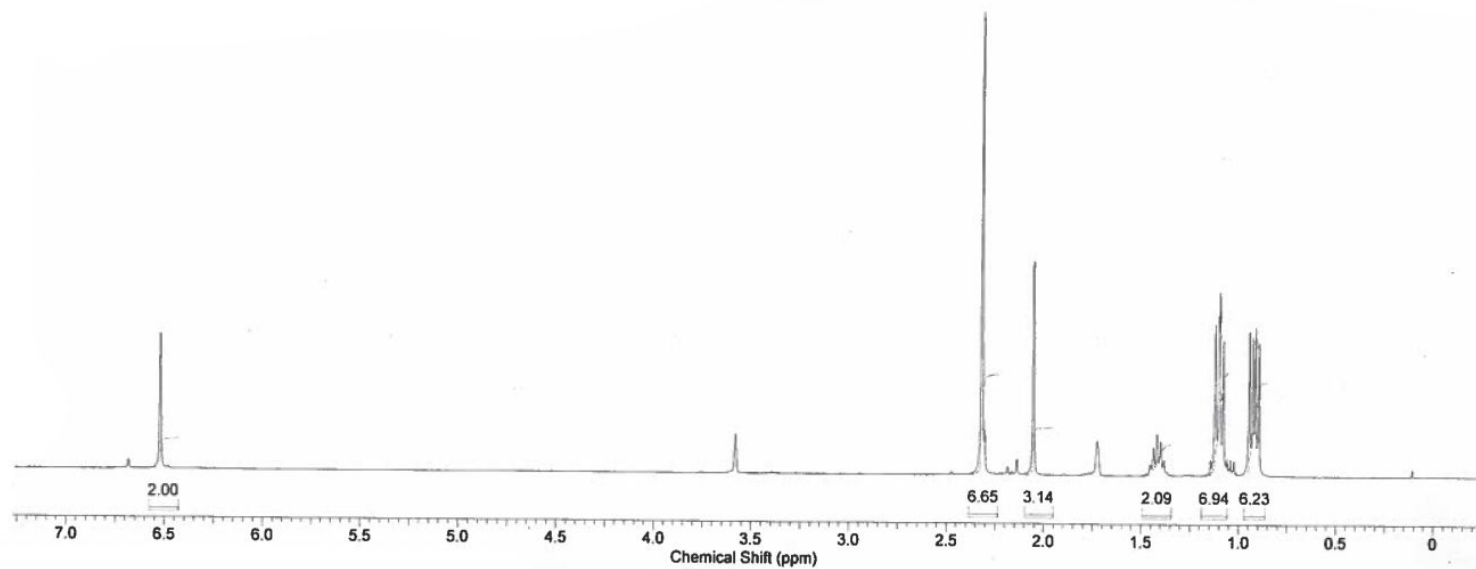

Figure S37. ${ }^{1} \mathrm{H}$ NMR spectrum of $\mathbf{S 2}$ in $\mathrm{D}_{8}$-THF. 


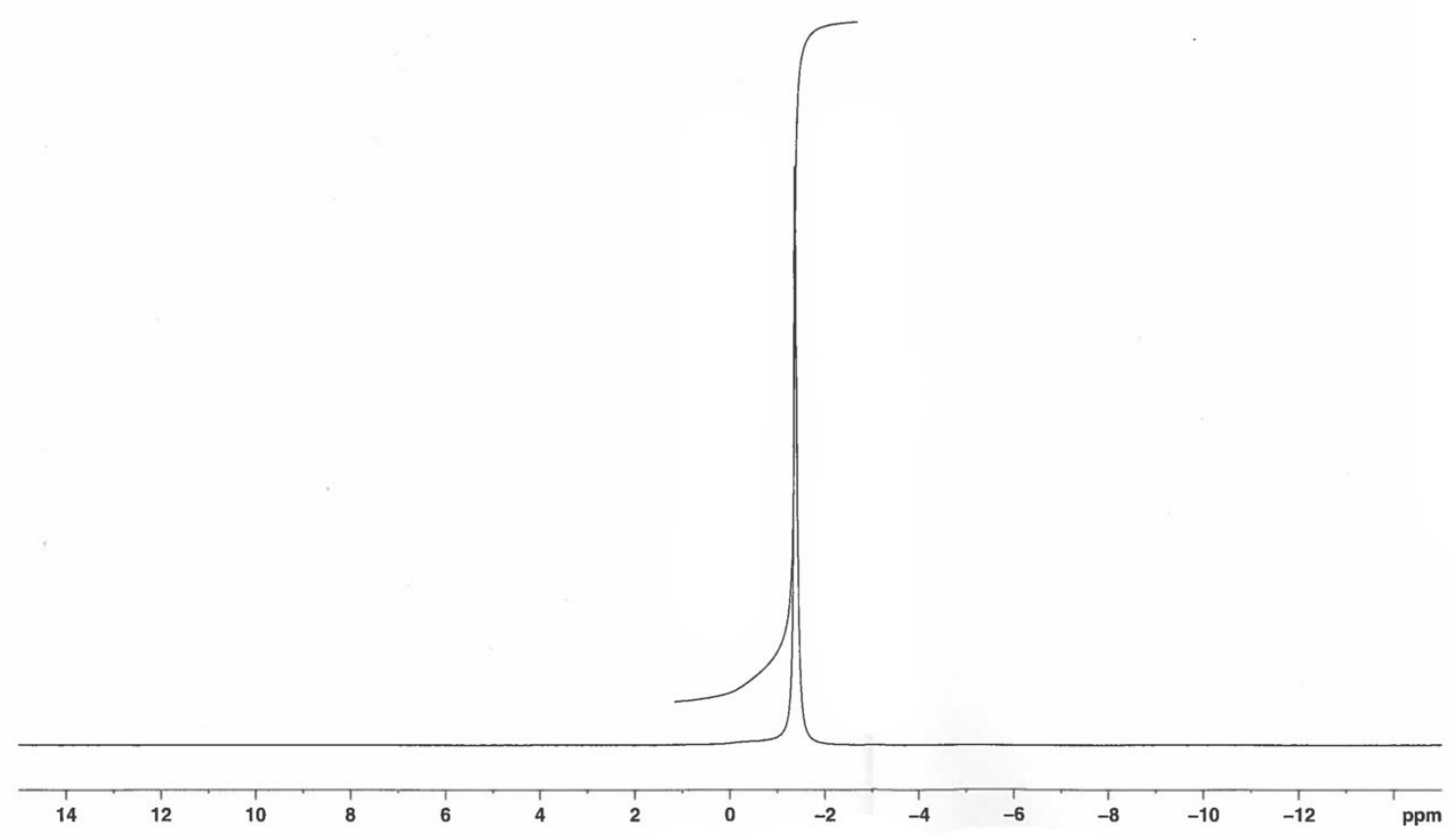

Figure S38. ${ }^{7} \mathrm{Li}$ NMR spectrum of $\mathbf{S} 2$ in $\mathrm{D}_{8}$-THF.

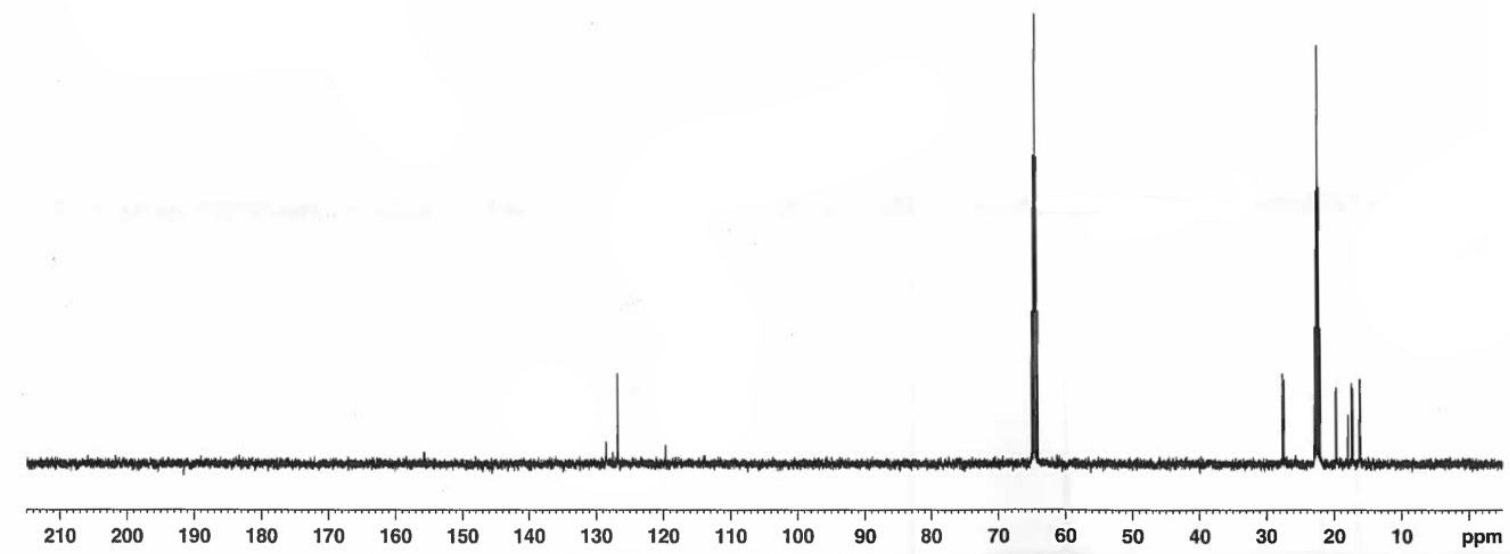

Figure S39. ${ }^{13} \mathrm{C}\left\{{ }^{1} \mathrm{H}\right\}$ NMR spectrum of $\mathbf{S 2}$ in $\mathrm{D}_{8}-\mathrm{THF}$. 


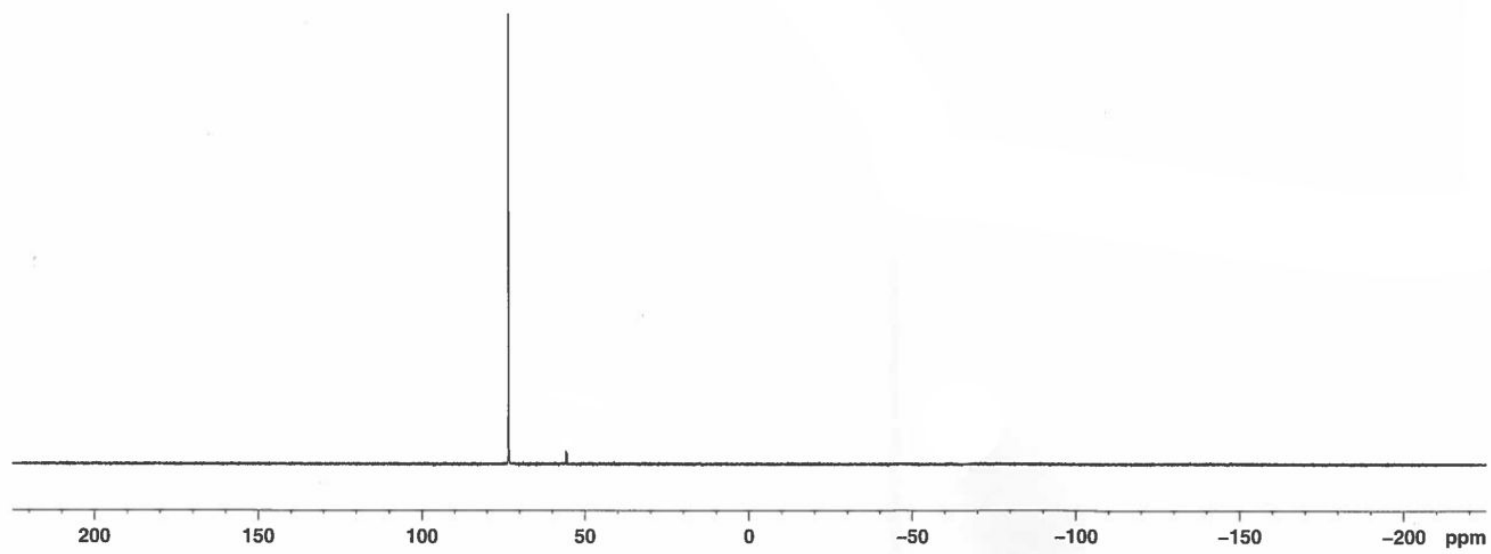

Figure $\mathbf{S 4 0} .{ }^{31} \mathrm{P}\left\{{ }^{1} \mathrm{H}\right\}$ NMR spectrum of $\mathbf{S} 2$ in $\mathrm{D}_{8}$-THF.

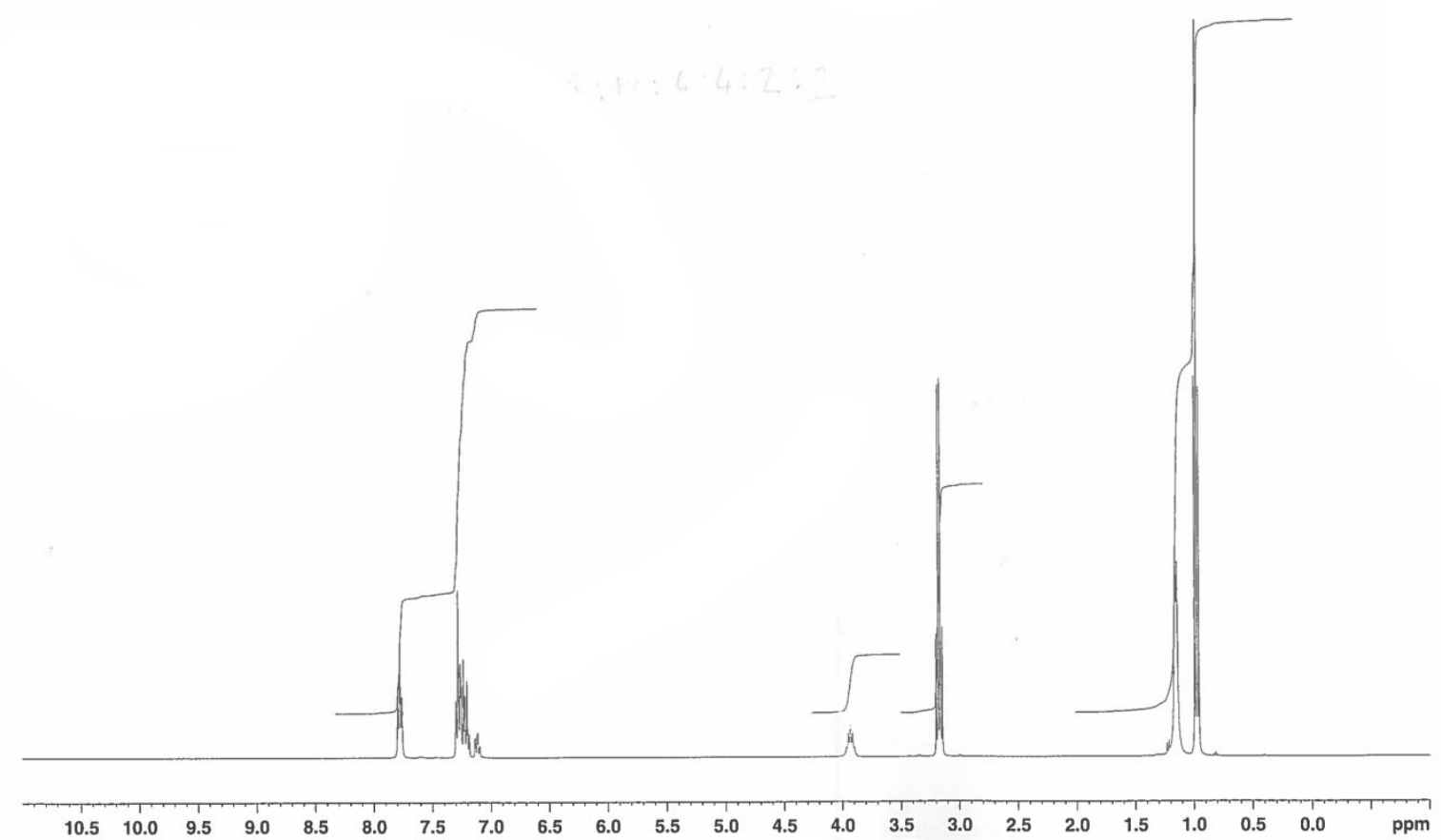

Figure S41. ${ }^{1} \mathrm{H}$ NMR spectrum of $\mathbf{S 3}$ in $\mathrm{C}_{6} \mathrm{D}_{6}$. 


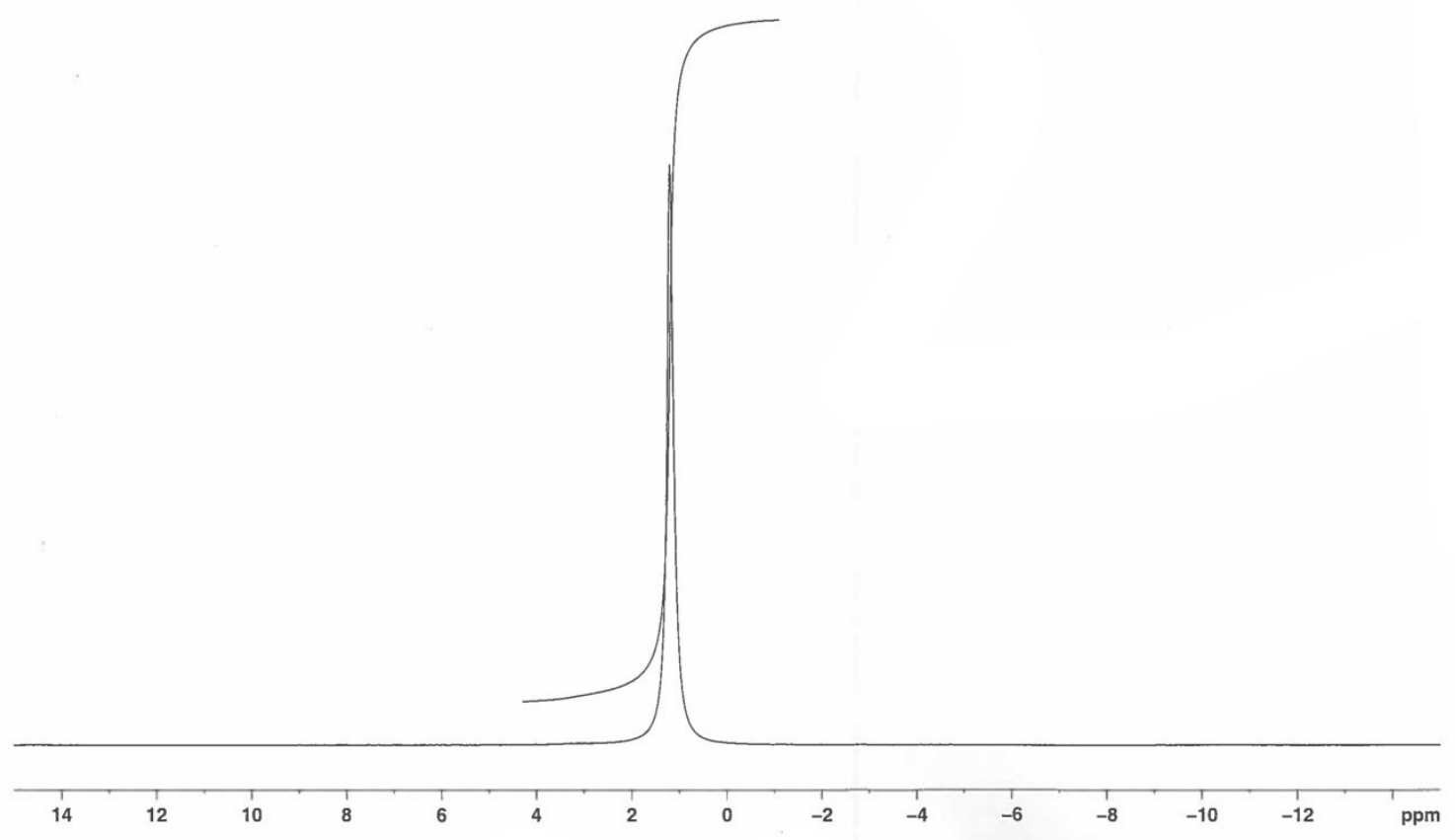

Figure $\mathbf{S 4 2 .}{ }^{7} \mathrm{Li}$ NMR spectrum of $\mathbf{S 3}$ in $\mathrm{C}_{6} \mathrm{D}_{6}$.

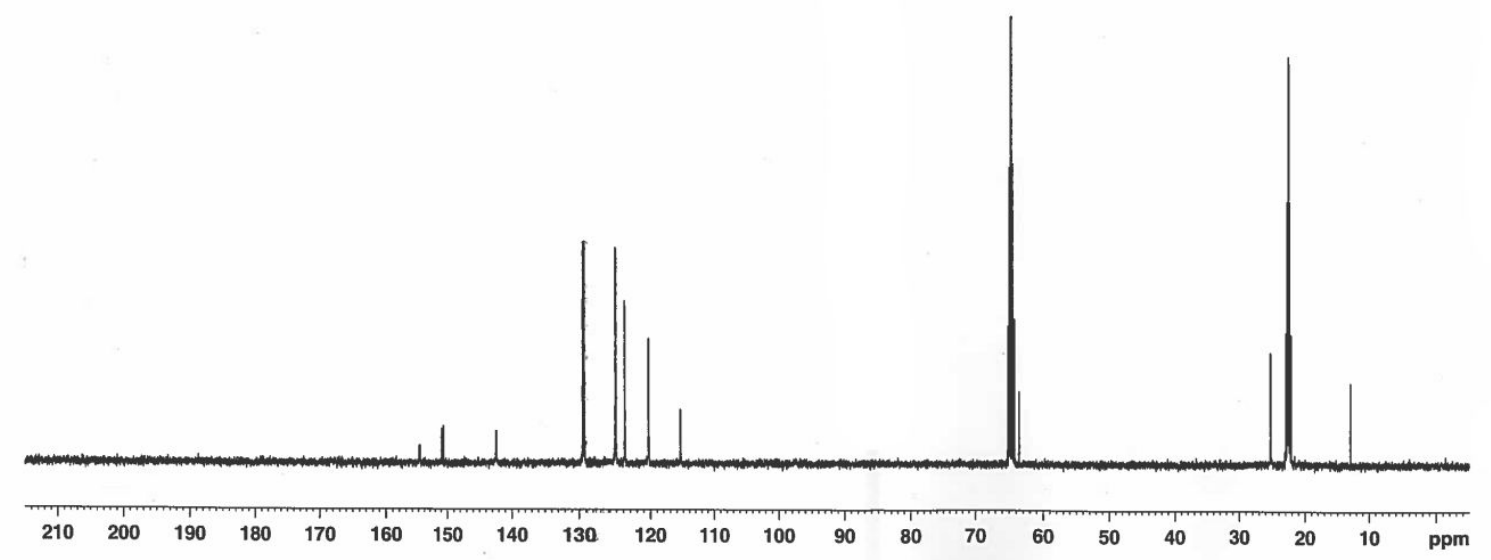

Figure S43. ${ }^{13} \mathrm{C}\left\{{ }^{1} \mathrm{H}\right\}$ NMR spectrum of $\mathbf{S 3}$ in $\mathrm{C}_{6} \mathrm{D}_{6}$. 


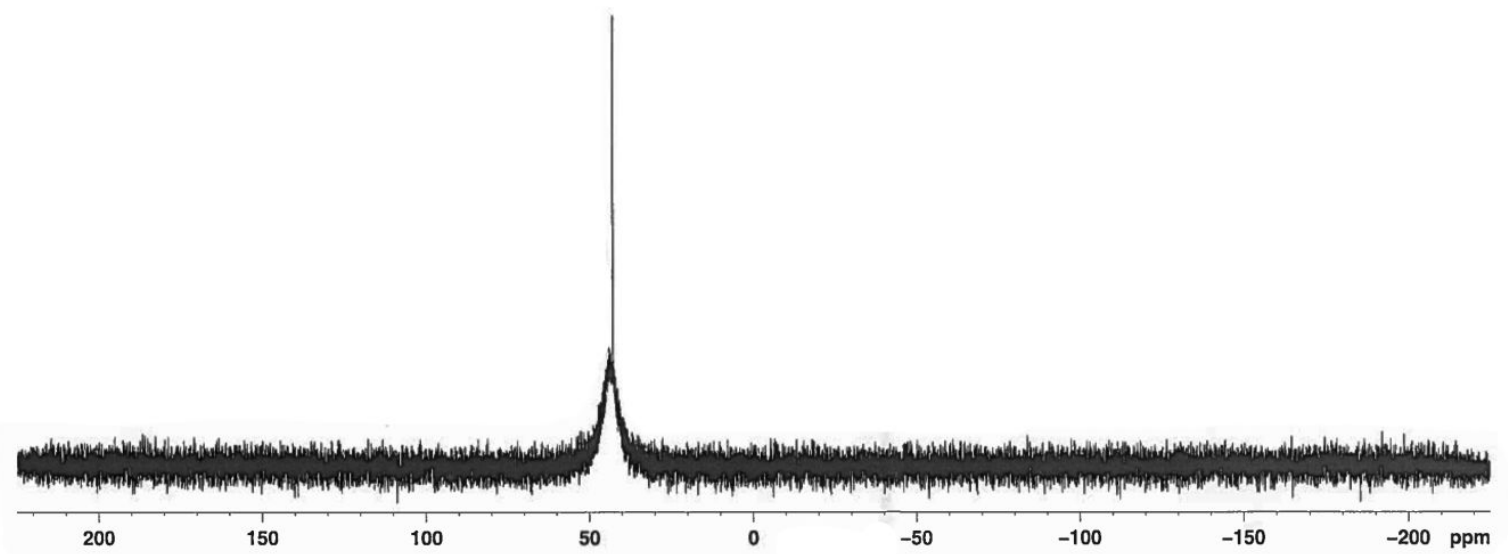

Figure S44. ${ }^{31} \mathrm{P}\left\{{ }^{1} \mathrm{H}\right\}$ NMR spectrum of $\mathbf{S 3}$ in $\mathrm{C}_{6} \mathrm{D}_{6}$.

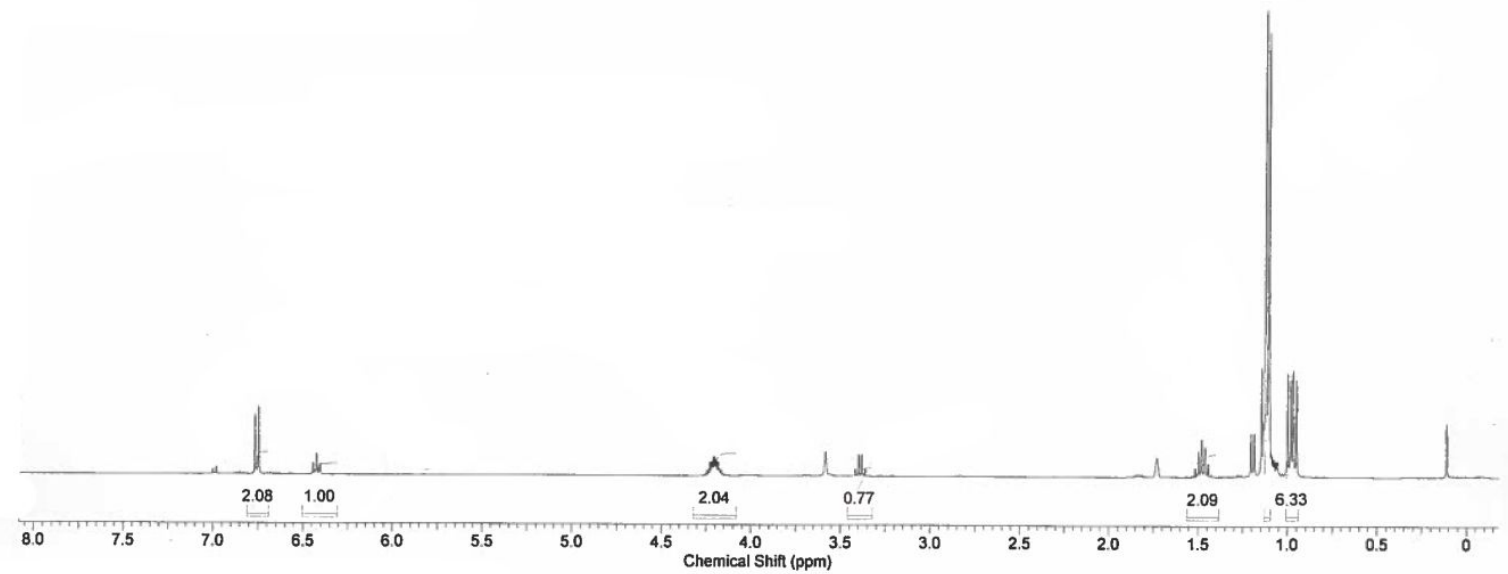

Figure S45. ${ }^{1} \mathrm{H}$ NMR spectrum of $\mathbf{S 4}$ in $\mathrm{D}_{8}$-THF. 


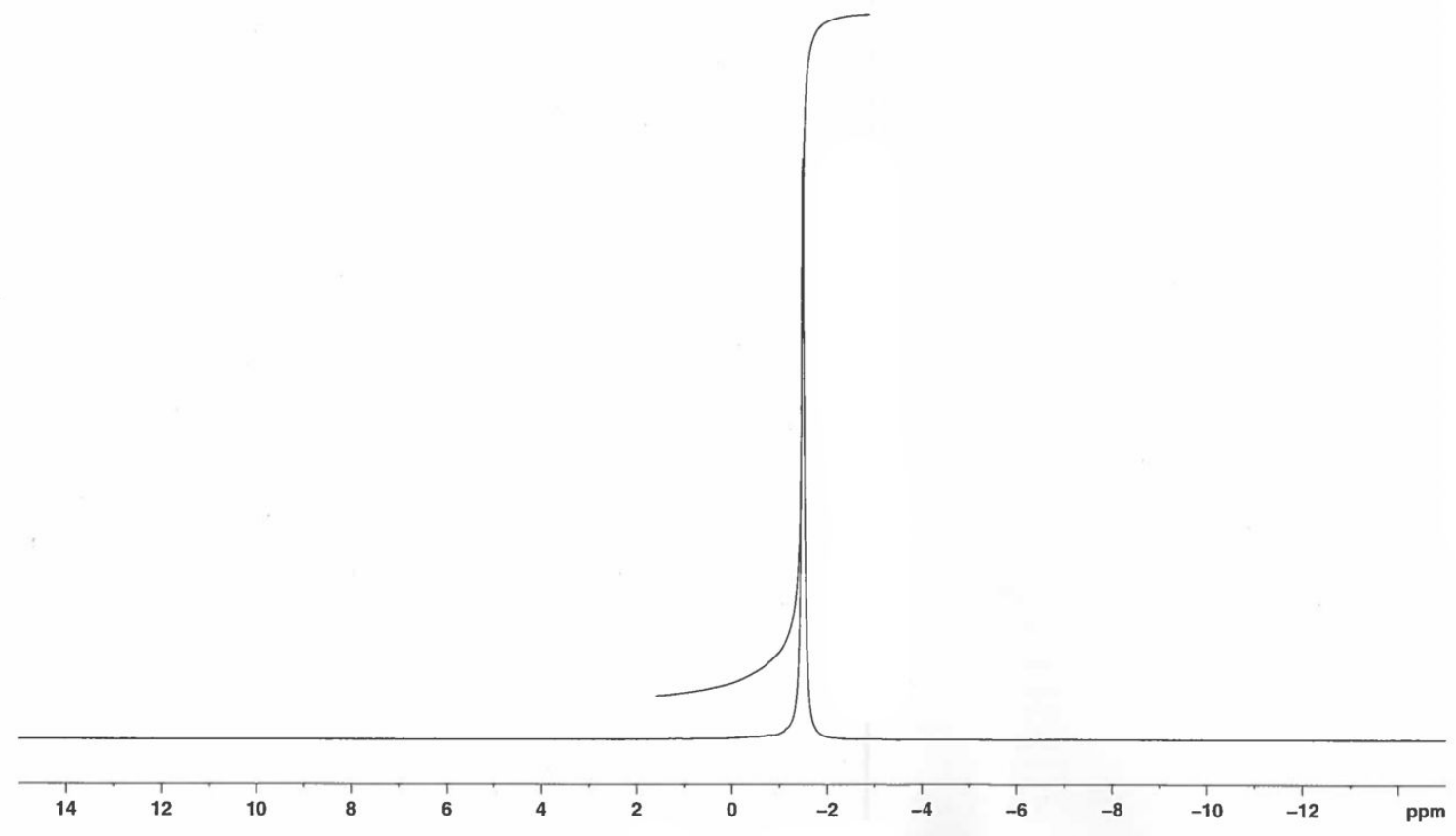

Figure $\mathbf{S 4 6} .{ }^{7} \mathrm{Li}$ NMR spectrum of $\mathbf{S} 4$ in $\mathrm{D}_{8}$-THF.

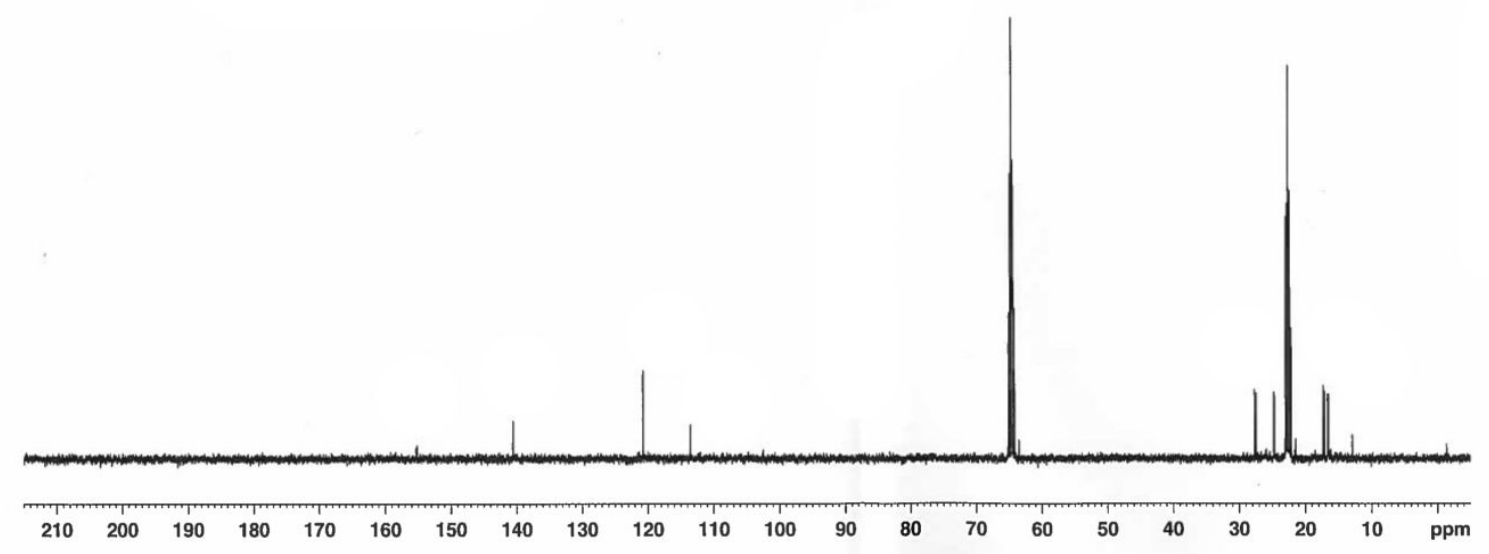

Figure $\mathbf{S 4 7 .}{ }^{13} \mathrm{C}\left\{{ }^{1} \mathrm{H}\right\}$ NMR spectrum of $\mathbf{S} 4$ in $\mathrm{D}_{8}$-THF. 


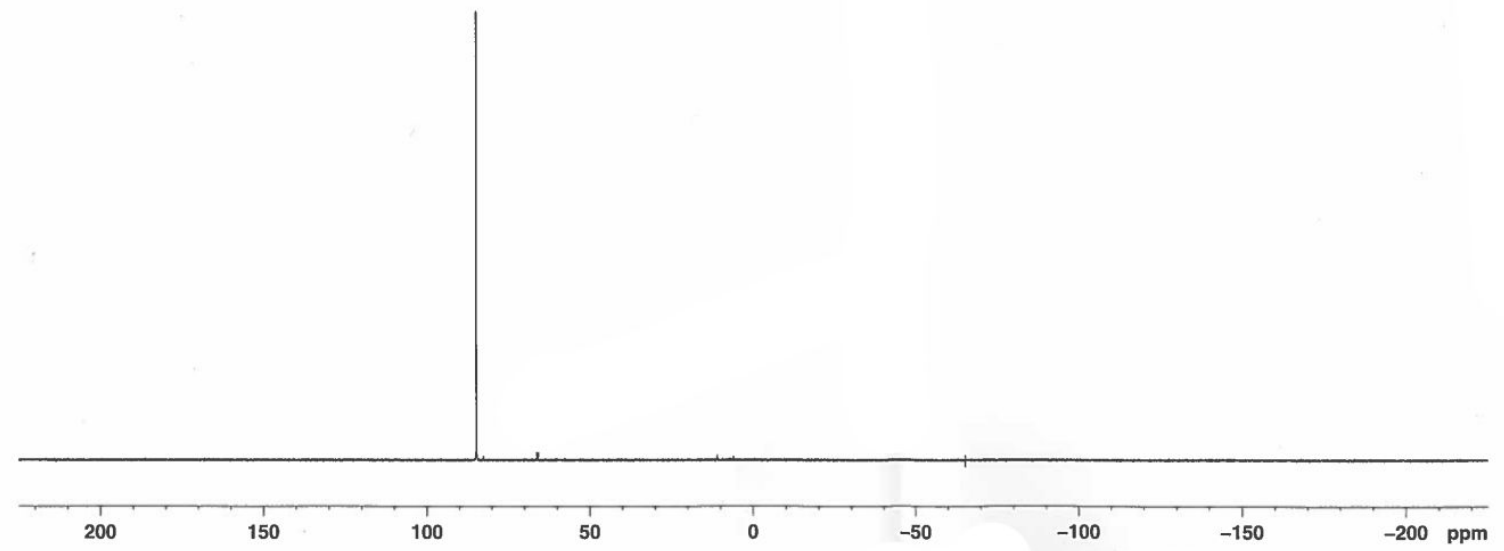

Figure $\mathbf{S 4 8 .}{ }^{31} \mathrm{P}\left\{{ }^{1} \mathrm{H}\right\}$ NMR spectrum of $\mathbf{S} 4$ in $\mathrm{D}_{8}$-THF.

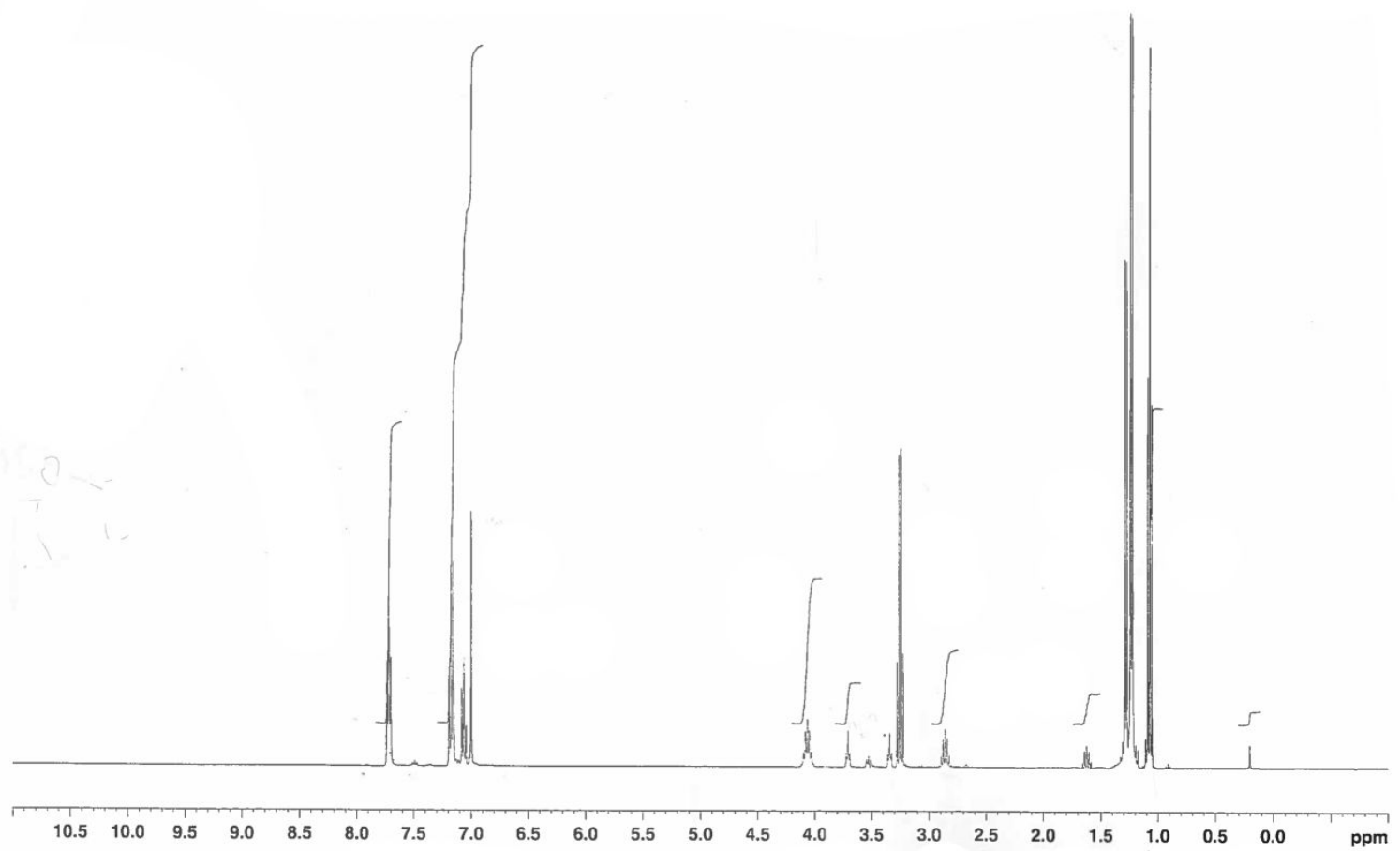

Figure S49. ${ }^{1} \mathrm{H}$ NMR spectrum of $\mathbf{S 5}$ in $\mathrm{C}_{6} \mathrm{D}_{6}$. 


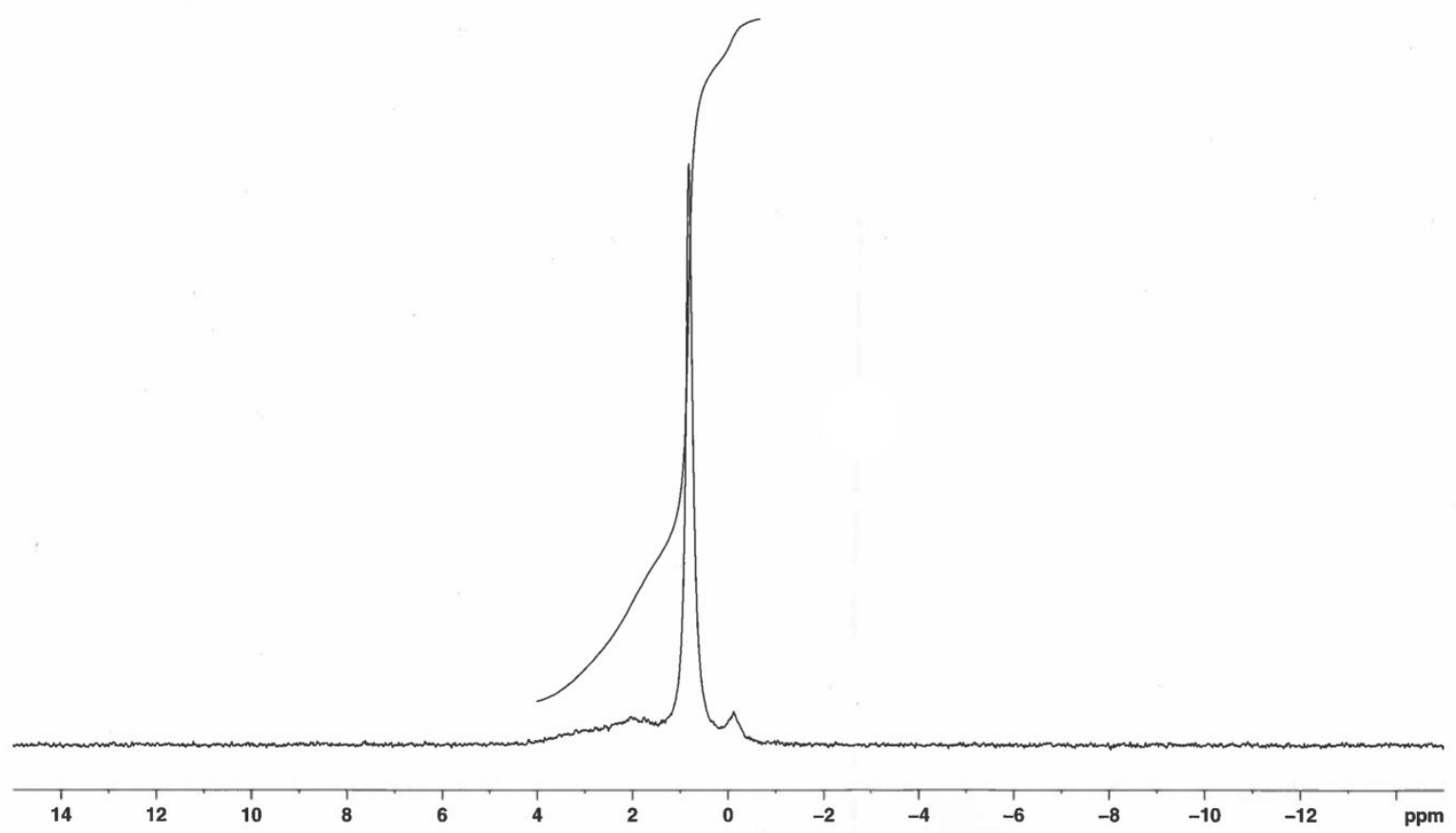

Figure S50. ${ }^{7} \mathrm{Li}$ NMR spectrum of $\mathbf{S 5}$ in $\mathrm{C}_{6} \mathrm{D}_{6}$.

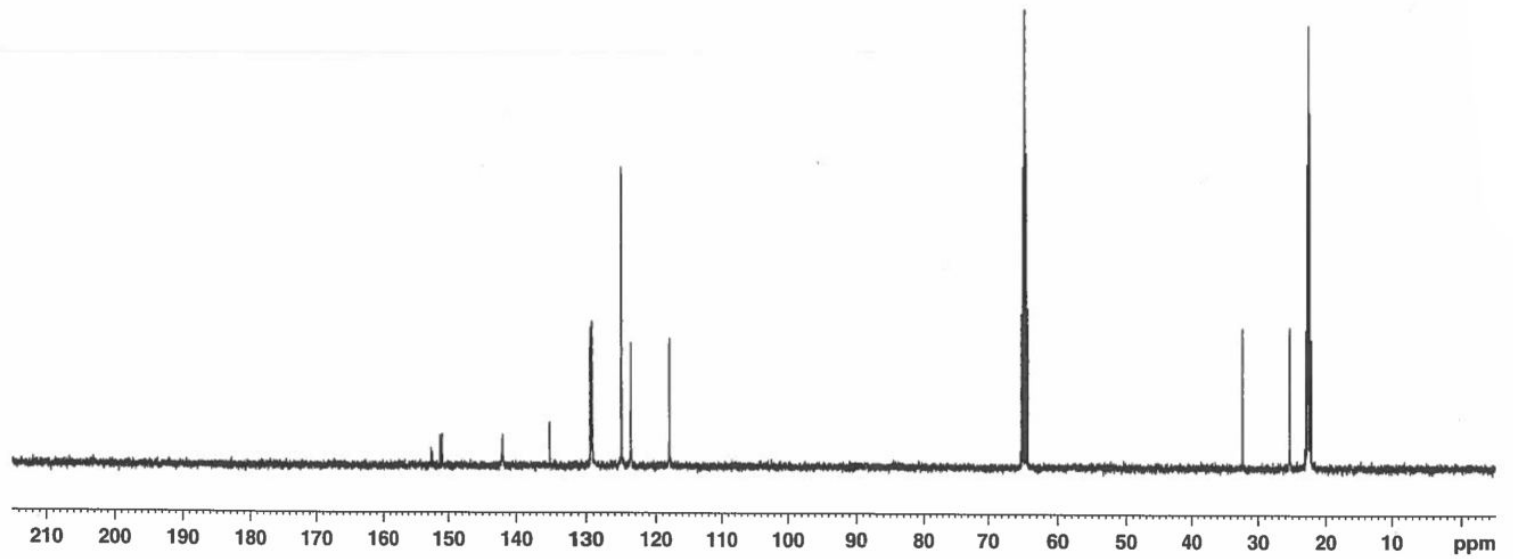

Figure S51. ${ }^{13} \mathrm{C}\left\{{ }^{1} \mathrm{H}\right\}$ NMR spectrum of $\mathbf{S 5}$ in $\mathrm{C}_{6} \mathrm{D}_{6}$. 


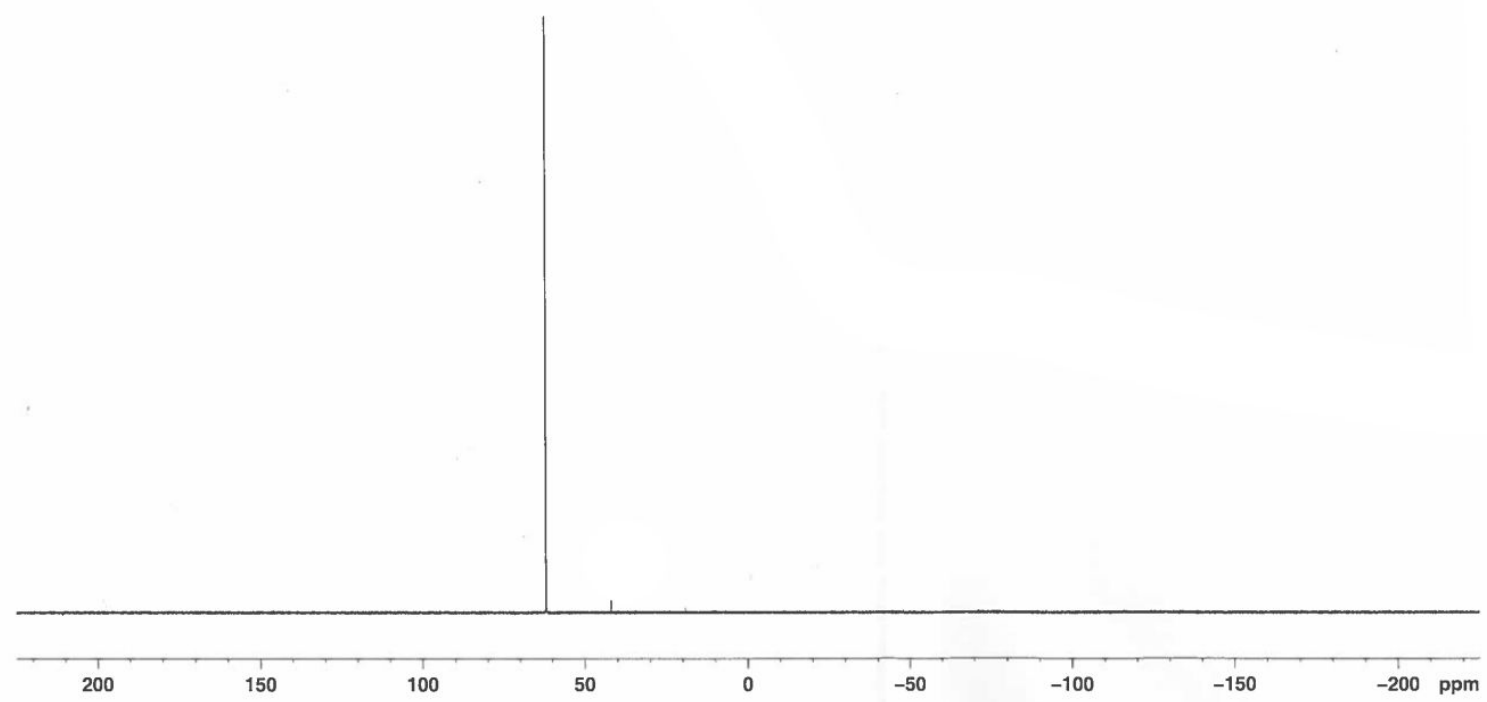

Figure S52. ${ }^{31} \mathrm{P}\left\{{ }^{1} \mathrm{H}\right\}$ NMR spectrum of $\mathbf{S 5}$ in $\mathrm{C}_{6} \mathrm{D}_{6}$.

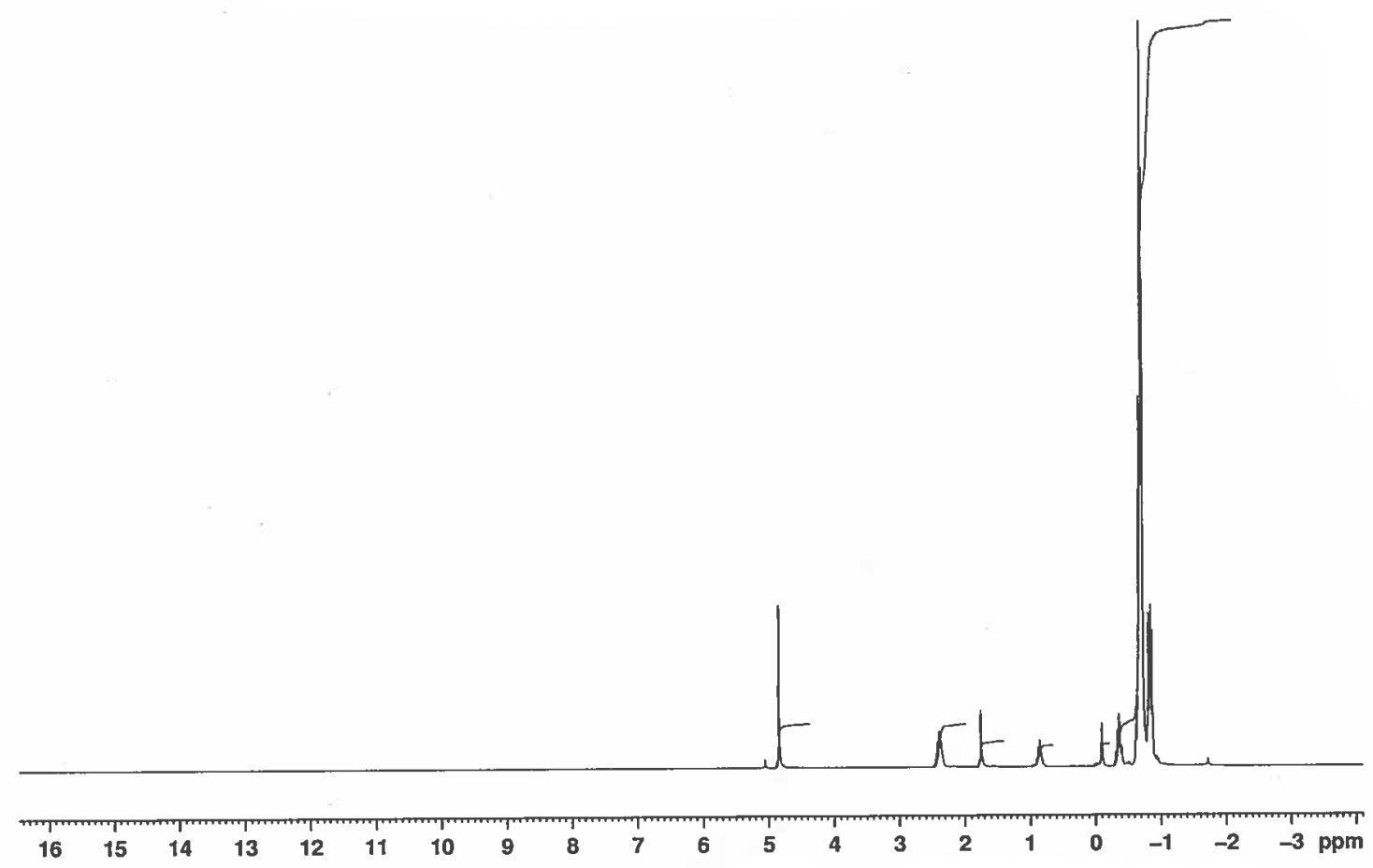

Figure S53. ${ }^{1} \mathrm{H}$ NMR spectrum of $\mathbf{S 6}$ in $\mathrm{D}_{8}$-THF. The printed resonances are relative to the incorrect residual THF resonance. The quoted values are referenced correctly. 


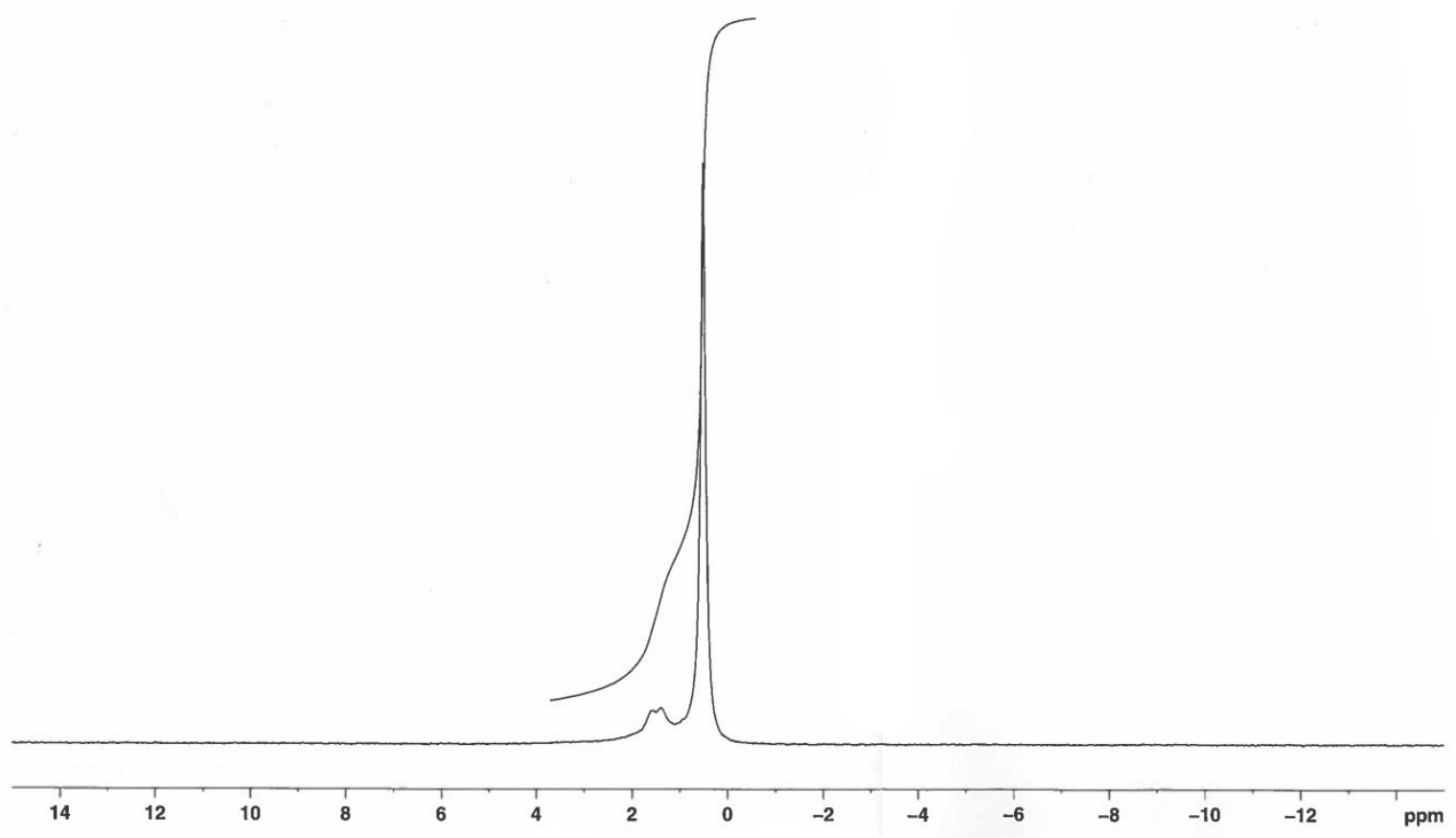

Figure S54. ${ }^{7} \mathrm{Li}\left\{{ }^{1} \mathrm{H}\right\}$ NMR spectrum of $\mathbf{S 6}$ in $\mathrm{D}_{8}$-THF.

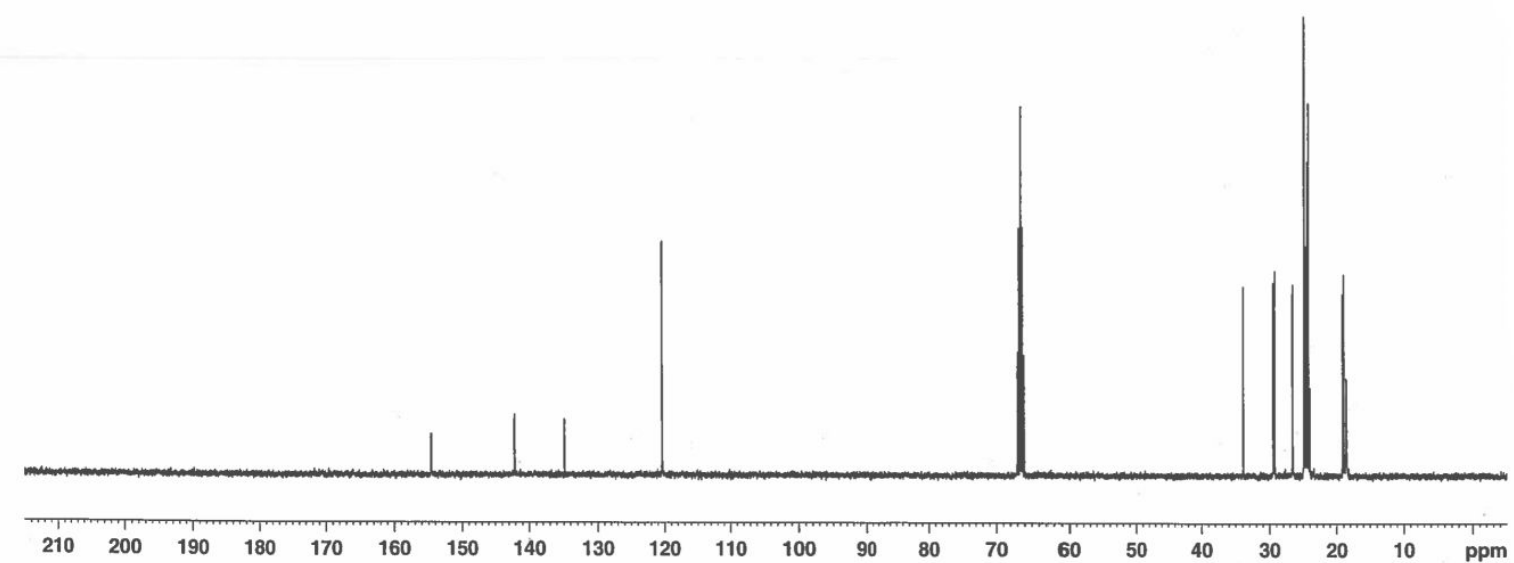

Figure S55. ${ }^{13} \mathrm{C}\left\{{ }^{1} \mathrm{H}\right\}$ NMR spectrum of $\mathbf{S 6}$ in $\mathrm{D}_{8}$-THF. 


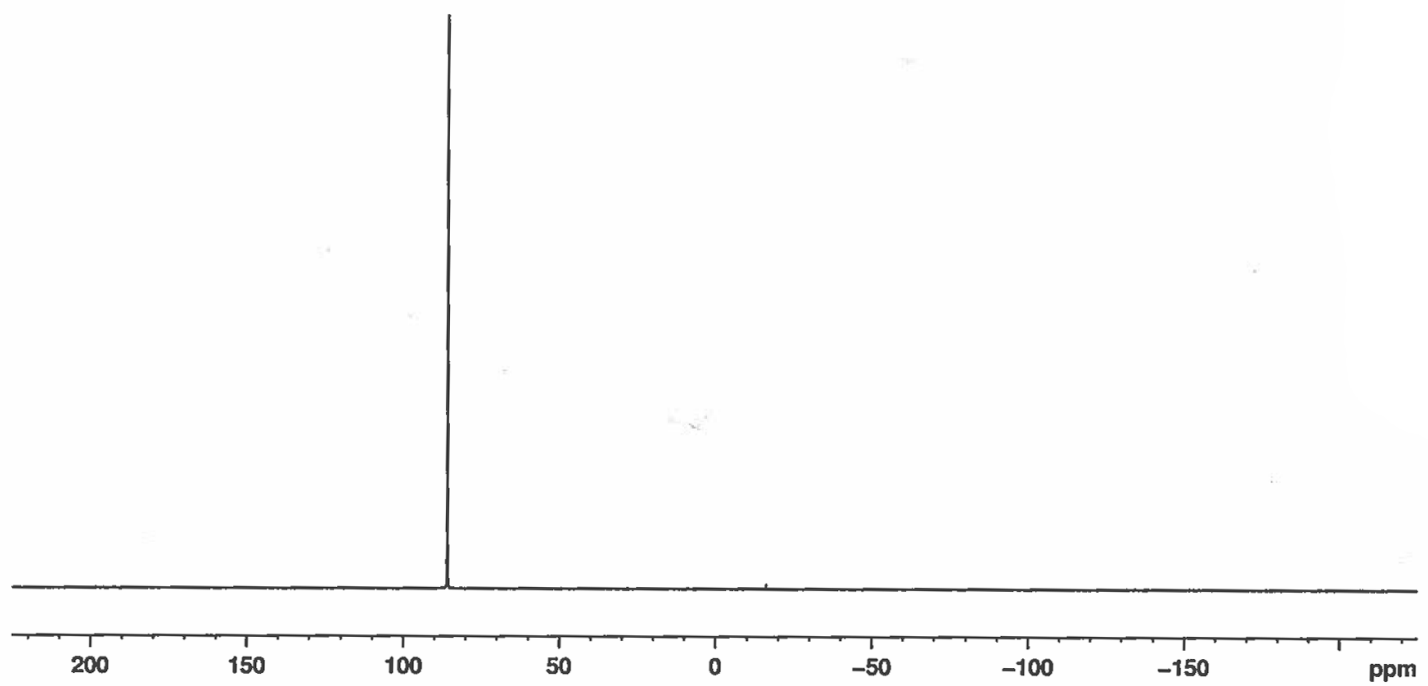

Figure S56. ${ }^{31} \mathrm{P}\left\{{ }^{1} \mathrm{H}\right\}$ NMR spectrum of $\mathbf{S 6}$ in $\mathrm{D}_{8}$-THF.

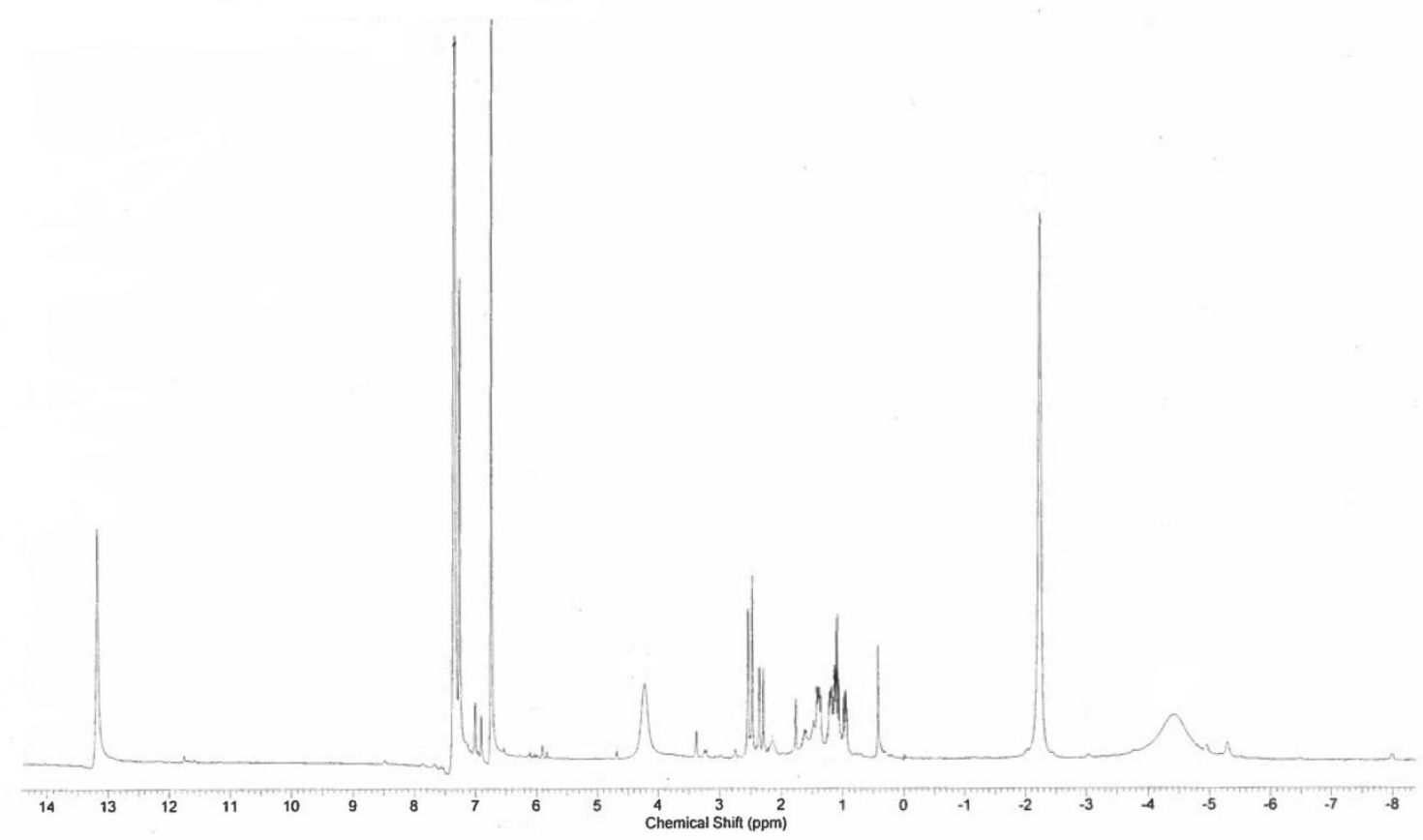

Figure S57. ${ }^{1} \mathrm{H}$ NMR spectrum of $\mathbf{S 8}$ in $\mathrm{C}_{6} \mathrm{D}_{6}$. 


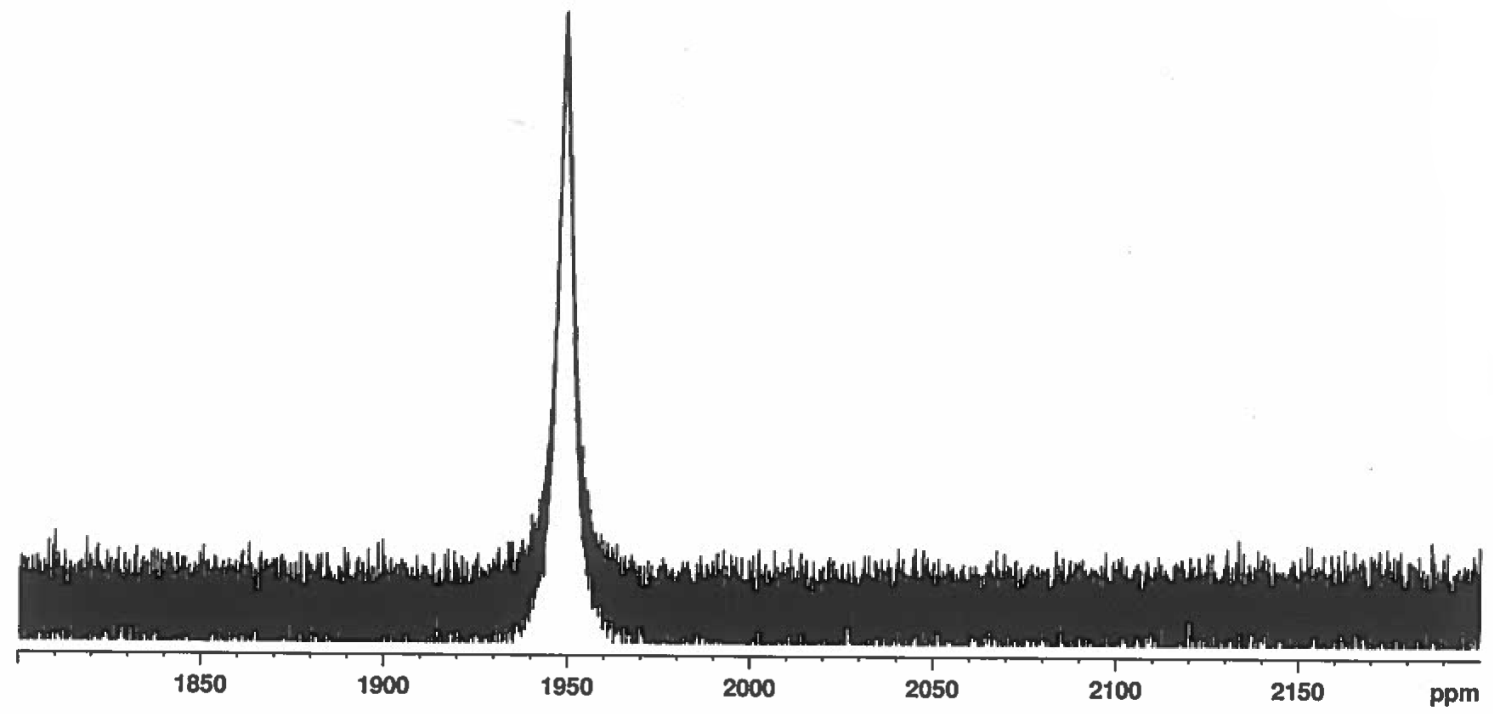

Figure S58. ${ }^{31} \mathrm{P}\left\{{ }^{1} \mathrm{H}\right\}$ NMR spectrum of $\mathbf{S 8}$ in $\mathrm{C}_{6} \mathrm{D}_{6}$.

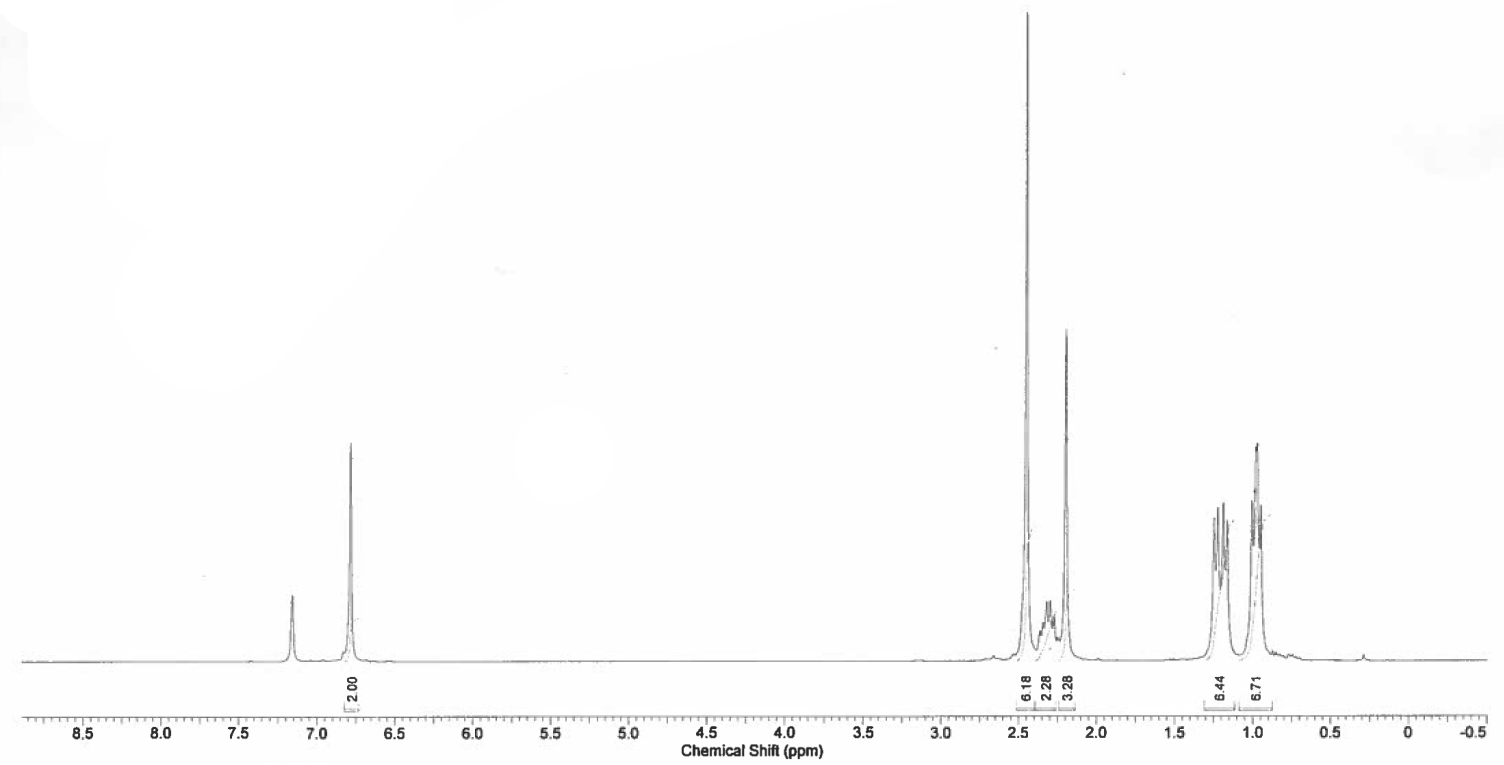

Figure S59. ${ }^{1} \mathrm{H}$ NMR spectrum of $\mathbf{S 9}$ in $\mathrm{C}_{6} \mathrm{D}_{6}$. 


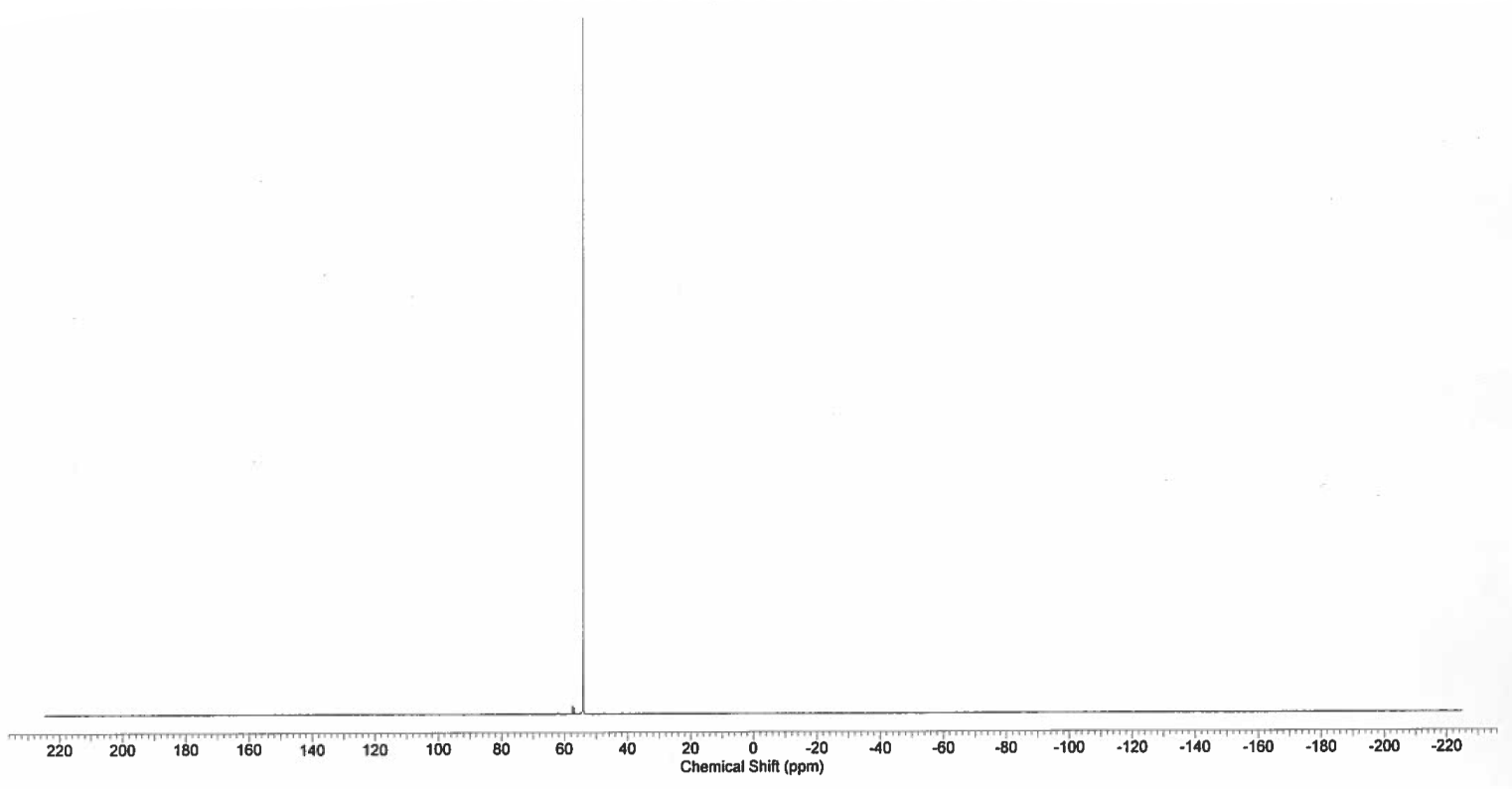

Figure S60. ${ }^{31} \mathrm{P}\left\{{ }^{1} \mathrm{H}\right\} \mathrm{NMR}$ spectrum of $\mathbf{S 9}$ in $\mathrm{C}_{6} \mathrm{D}_{6}$.

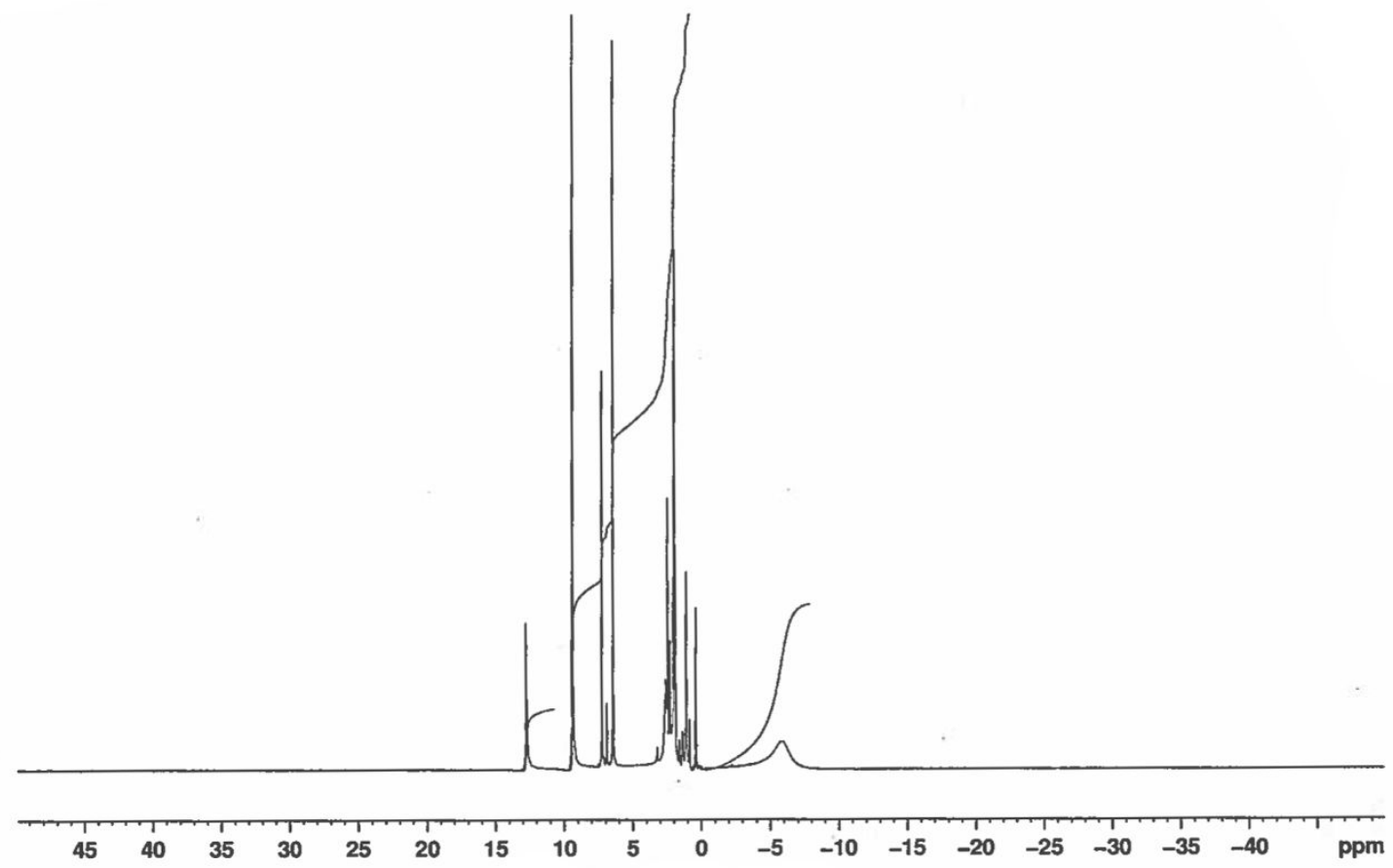

Figure S61. ${ }^{1} \mathrm{H}$ NMR spectrum of $\mathbf{S 1 0}$ in $\mathrm{C}_{6} \mathrm{D}_{6}$. 


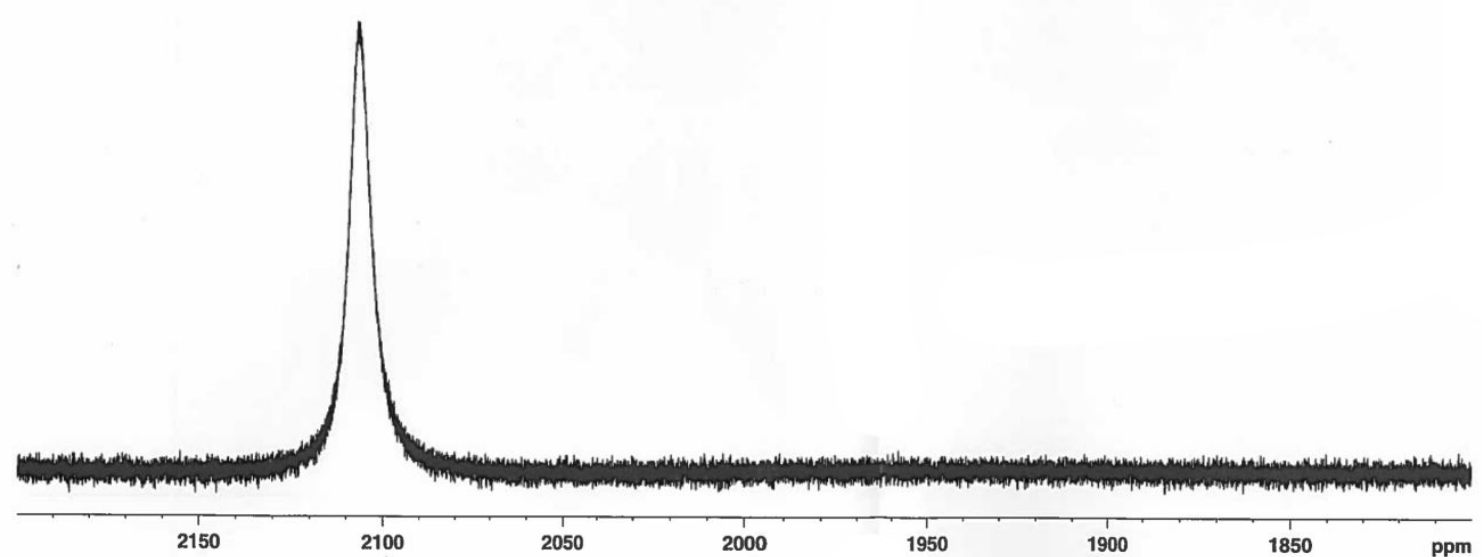

Figure S62. ${ }^{31} \mathrm{P}\left\{{ }^{1} \mathrm{H}\right\}$ NMR spectrum of $\mathbf{S 1 0}$ in $\mathrm{C}_{6} \mathrm{D}_{6}$.

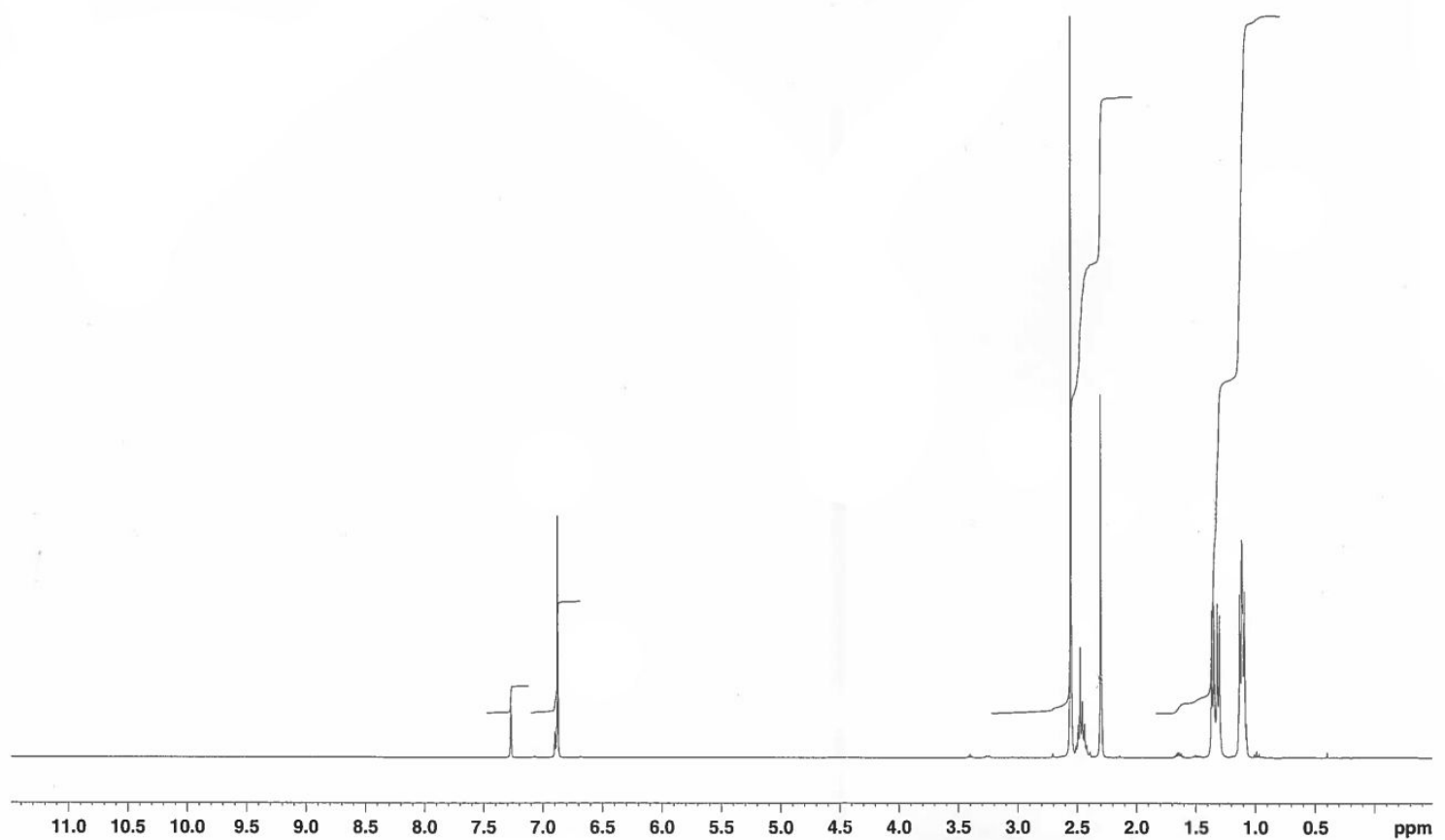

Figure S63. ${ }^{1} \mathrm{H}$ NMR spectrum of $\mathbf{S 1 1}$ in $\mathrm{C}_{6} \mathrm{D}_{6}$. The resonance at $7.27 \mathrm{ppm}$ is residual benzene and should be $7.16 \mathrm{ppm}$, but the assigned resonances are quoted relative to the correct reference value. 


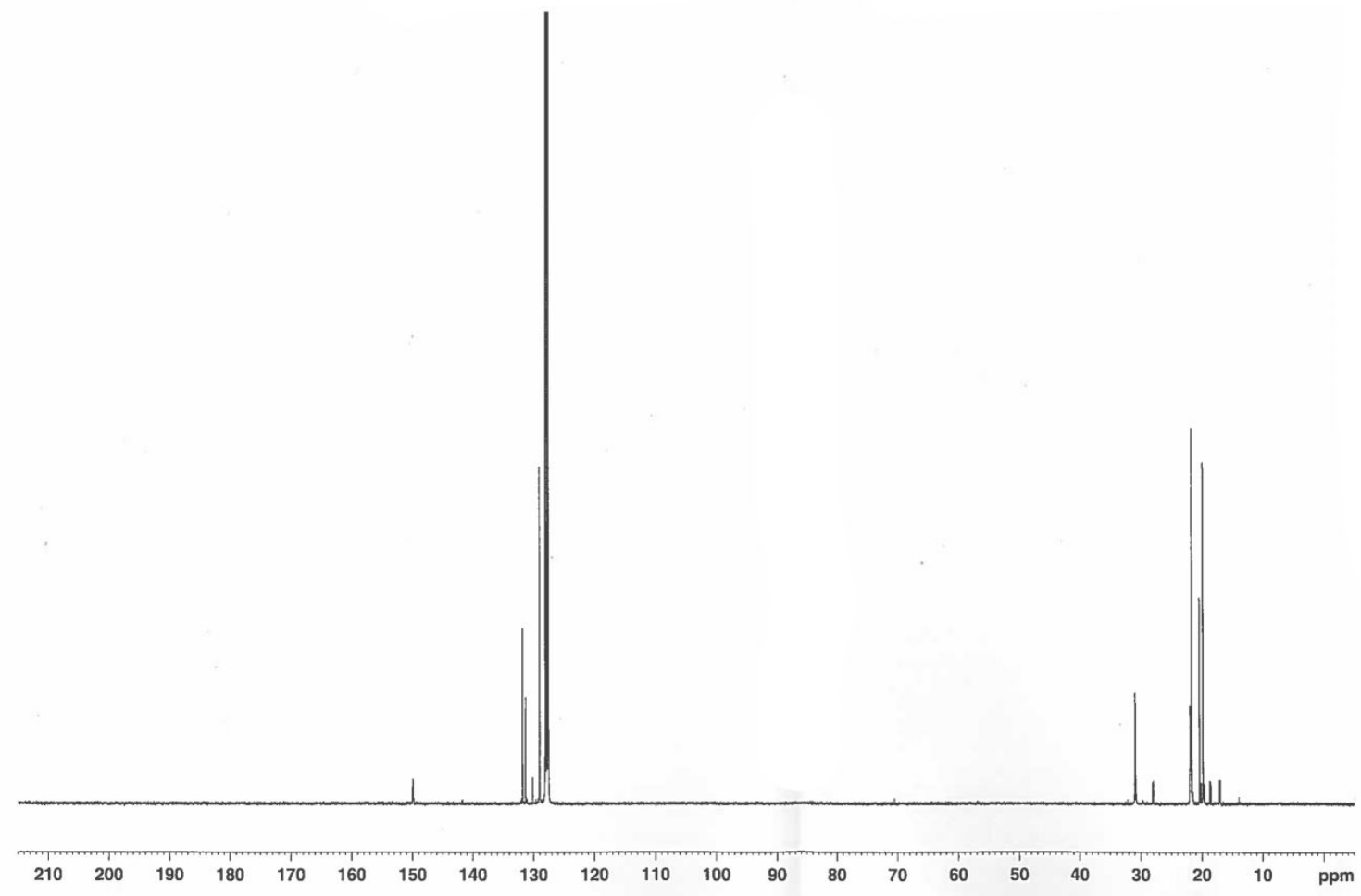

Figure S64. ${ }^{13} \mathrm{C}\left\{{ }^{1} \mathrm{H}\right\}$ NMR spectrum of $\mathbf{S 1 1}$ in $\mathrm{C}_{6} \mathrm{D}_{6}$.

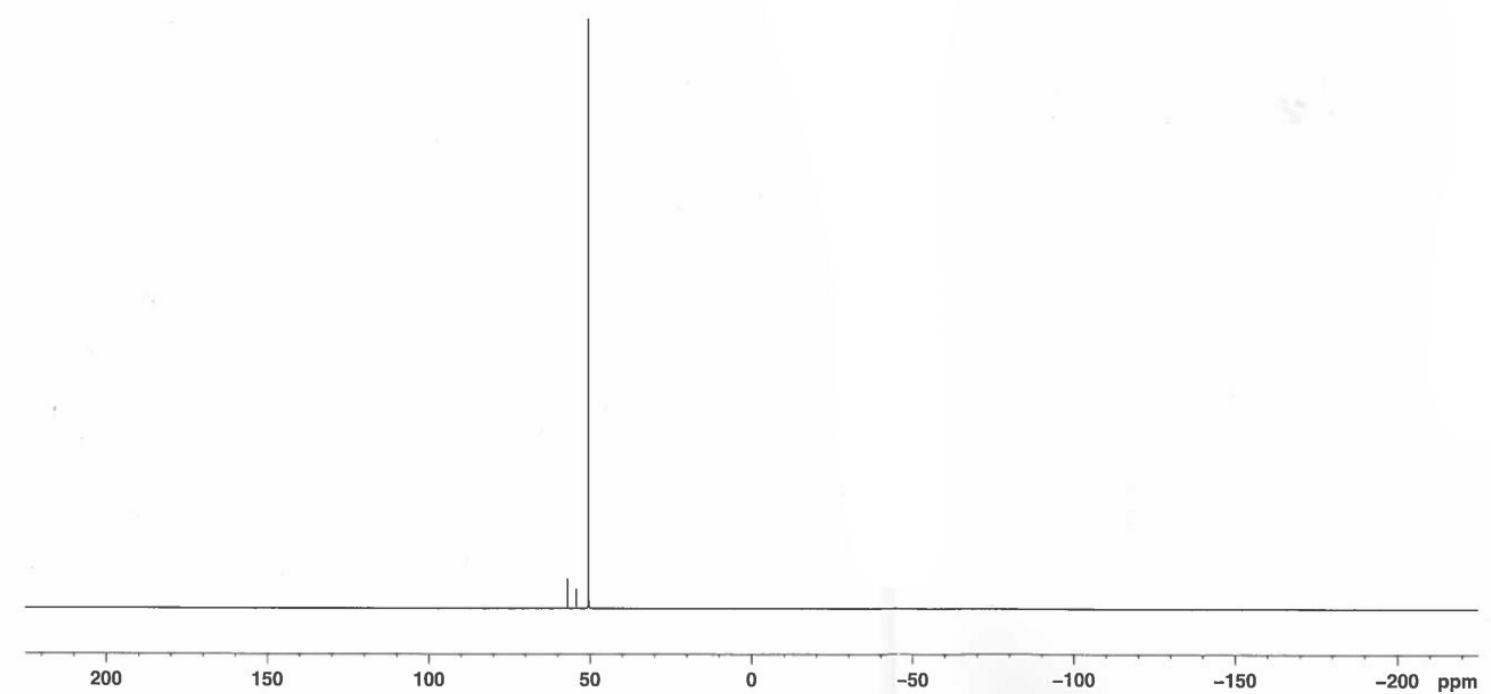

Figure S65. ${ }^{31} \mathrm{P}\left\{{ }^{1} \mathrm{H}\right\}$ NMR spectrum of $\mathbf{S 1 1}$ in $\mathrm{C}_{6} \mathrm{D}_{6}$. 


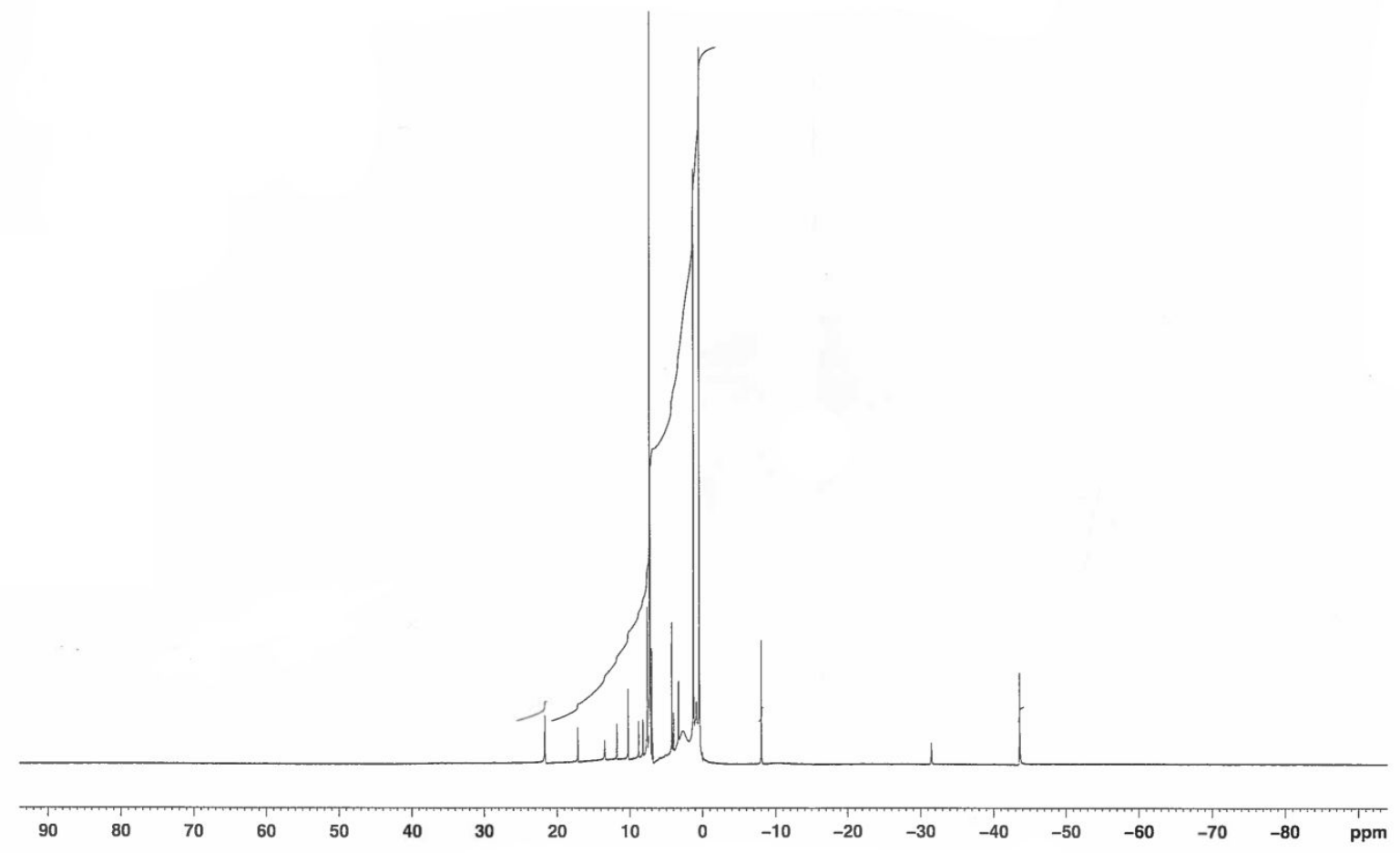

Figure S66. ${ }^{1} \mathrm{H}$ NMR spectrum of $\mathbf{S 1 2}$ in $\mathrm{C}_{6} \mathrm{D}_{6}$.

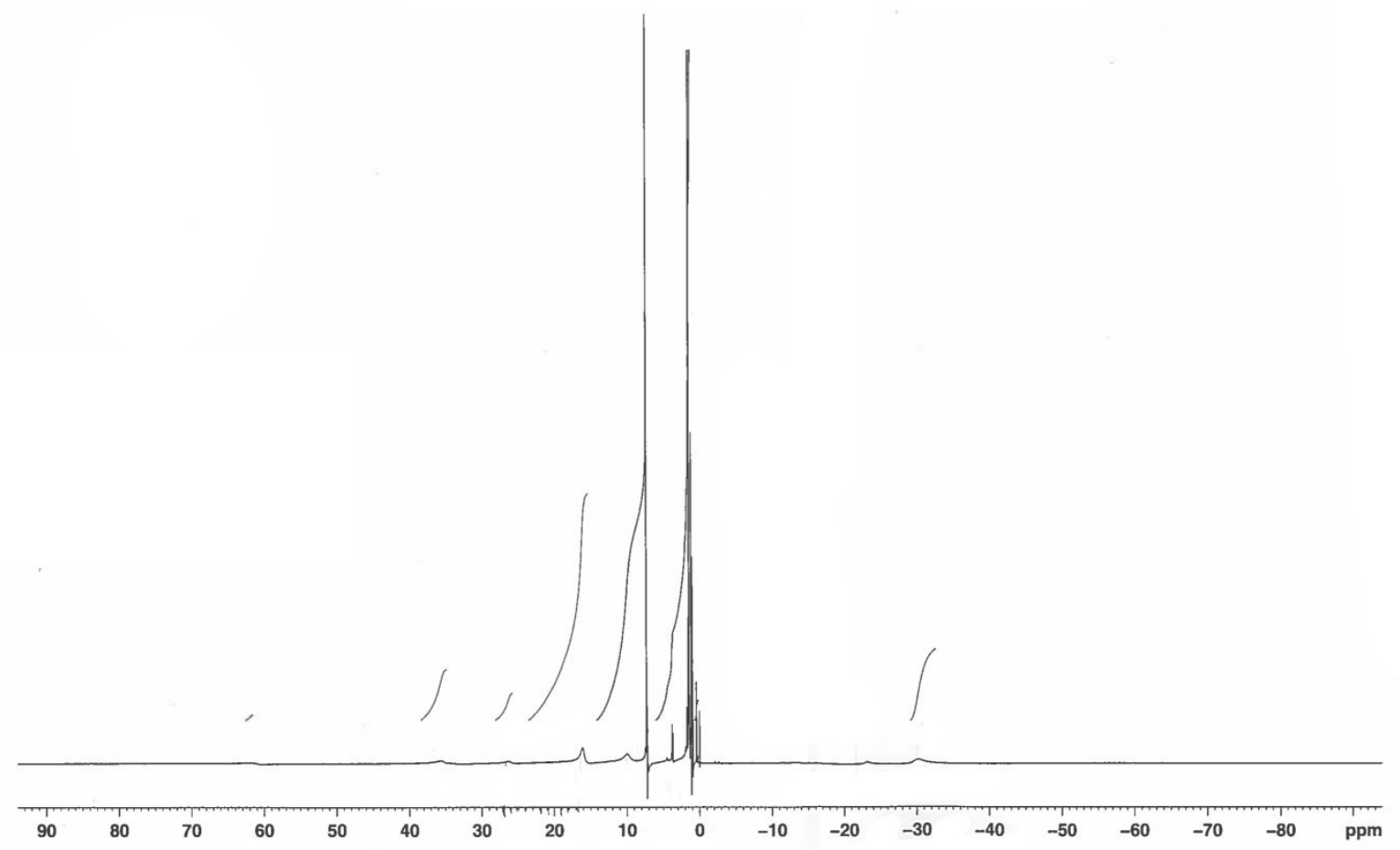

Figure S67. ${ }^{1} \mathrm{H}$ NMR spectrum of $\mathbf{S 1 3}$ in $\mathrm{C}_{6} \mathrm{D}_{6}$. 


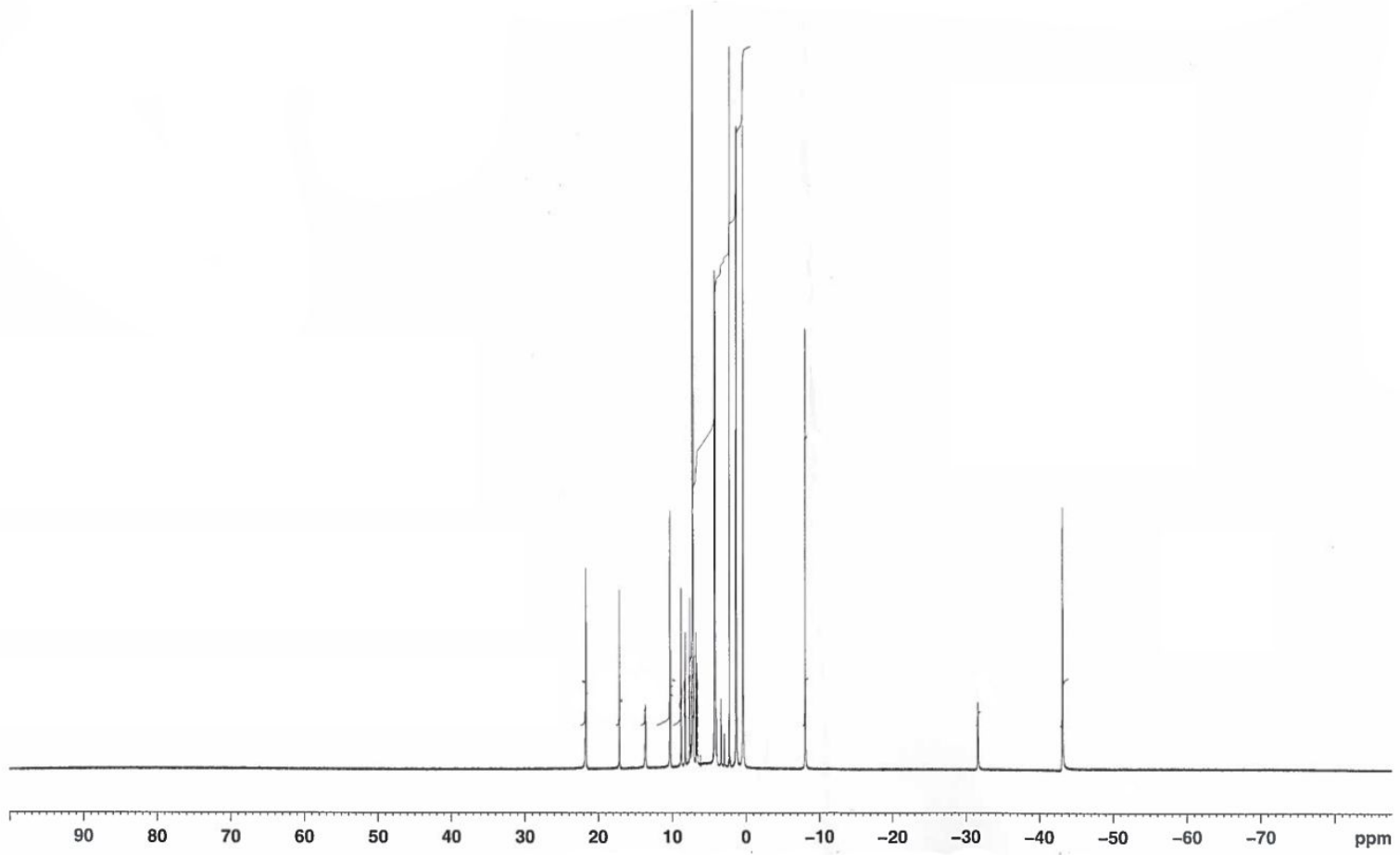

Figure S68. ${ }^{1} \mathrm{H}$ NMR spectrum of $\mathbf{S 1 4}$ in $\mathrm{C}_{6} \mathrm{D}_{6}$. 
Table S1. Crystallographic data for compounds 1-4. ${ }^{a}$ Conventional $R=\Sigma|| F_{\mathrm{o}}|-| F \mathrm{c}|| / \Sigma\left|F_{\mathrm{O}}\right| ; R_{\mathrm{w}}=$ $\left[\Sigma \mathrm{w}\left(F_{\mathrm{o}}^{2}-F_{\mathrm{c}}^{2}\right)^{2} / \Sigma \mid\left(F_{\mathrm{o}}^{2}\right)^{2}\right]^{1 / 2} ; S=\left[\Sigma \mathrm{w}\left(F_{\mathrm{o}}^{2}-F_{\mathrm{c}}^{2}\right)^{2} /(\text { no. data }- \text { no. parameters })\right]^{1 / 2}$ for all data.

\begin{tabular}{|c|c|c|c|c|}
\hline & 1 & 2 & 3 & 4 \\
\hline Formula & $\mathrm{C}_{64} \mathrm{H}_{106} \mathrm{ClMoN}_{4} \mathrm{O}_{3} \mathrm{P}$ & $\begin{array}{l}\mathrm{C}_{62} \mathrm{H}_{90} \mathrm{ClMoN}_{4} \mathrm{O}_{3} \mathrm{P}_{3} \\
\text { Th }\end{array}$ & $\begin{array}{l}\mathrm{C}_{71} \mathrm{H}_{72} \mathrm{Cl}_{7} \mathrm{MoN}_{4} \mathrm{O}_{3} \mathrm{P} \\
{ }_{3} \mathrm{U}\end{array}$ & $\begin{array}{l}\mathrm{C}_{71} \mathrm{H}_{72} \mathrm{Cl}_{7} \mathrm{MoN}_{4} \mathrm{O}_{3} \mathrm{P}_{3} \\
\text { Th }\end{array}$ \\
\hline $\mathrm{Fw}, \mathrm{g} \mathrm{mol}^{-1}$ & 1441.85 & 1395.71 & 1704.35 & 1698.36 \\
\hline Cryst size, mm & $\begin{array}{llll}0.194 & \mathrm{x} & 0.113 & \mathrm{x} \\
0.050 & & & \end{array}$ & $\begin{array}{llll}0.211 & \mathrm{x} & 0.181 & \mathrm{x} \\
0.113 & & & \end{array}$ & $\begin{array}{llll}0.202 & \mathrm{x} & 0.176 & \mathrm{x} \\
0.0840 & & & \end{array}$ & $\begin{array}{llll}0.136 & \mathrm{x} & 0.096 & \mathrm{x} \\
0.060 & & & \end{array}$ \\
\hline Crystal system & orthorhombic & monoclinic & triclinic & triclinic \\
\hline Space group & $P$ bca & $P 2_{1} / \mathrm{n}$ & $P-1$ & $P-1$ \\
\hline $\begin{array}{l}\text { Collection } \\
\text { Temperature (K) }\end{array}$ & $120(2)$ & $120(2)$ & $120(2)$ & $120(2)$ \\
\hline $\mathrm{a},(\AA)$ & $23.2497(3)$ & $12.0815(19)$ & $13.5081(3)$ & $13.5262(3)$ \\
\hline $\mathrm{b},(\AA)$ & $18.2459(3)$ & $10.938(2)$ & 13.7881(4) & $13.8345(5)$ \\
\hline$c,(\AA)$ & $31.8905(5)$ & $46.726(4)$ & $20.4975(4)$ & $20.4136(6)$ \\
\hline$\alpha,\left(^{\circ}\right)$ & 90 & 90 & 79.3405(19) & $79.099(3)$ \\
\hline$\beta,\left(^{\circ}\right)$ & 90 & $95.102(10)$ & $87.0853(18)$ & $86.939(2)$ \\
\hline$\gamma,\left(^{\circ}\right)$ & 90 & 90 & $68.582(2)$ & $68.925(3)$ \\
\hline $\mathrm{V},\left(\AA^{3}\right)$ & $13528.3(4)$ & $6150.0(18)$ & $3492.12(16)$ & $3499.7(2)$ \\
\hline $\mathrm{Z}$ & 8 & 4 & 2 & 2 \\
\hline$\rho_{\text {calc }} \mathrm{g} \mathrm{cm}^{3}{ }^{3}$ & 1.416 & 1.507 & 1.621 & 1.612 \\
\hline$\mu, \mathrm{mm}^{-1}$ & 9.574 & 10.908 & 11.452 & 11.770 \\
\hline $\begin{array}{l}\text { No. of reflections } \\
\text { measured }\end{array}$ & 75510 & 10851 & 26477 & 26472 \\
\hline $\begin{array}{l}\text { No. of unique } \\
\text { reflections, Rint }\end{array}$ & $13703,0.0762$ & $10851, ?$ & $13800,0.0455$ & $13780,0.0353$ \\
\hline $\begin{array}{l}\text { No. of reflections } \\
\text { with } F^{2}>2 \mathrm{~s}\left(F^{2}\right)\end{array}$ & 11830 & 9682 & 13282 & 12842 \\
\hline $\begin{array}{l}\text { Transmission } \\
\text { coefficient range }\end{array}$ & $0.848-1.051$ & $0.208-0.417$ & $0.461-0.816$ & $0.366-0.600$ \\
\hline $\begin{array}{lll}R, \quad R_{\mathrm{w}}{ }^{a} & \left(F^{2} \quad>\right. \\
\left.2 \mathrm{~s}\left(F^{2}\right)\right) & & \\
\end{array}$ & $0.0530,0.1427$ & $0.1313,0.3425$ & $0.0381,0.0948$ & $0.0334,0.0875$ \\
\hline$R, R_{\mathrm{w}}{ }^{a}$ (all data) & $0.0608,0.1499$ & $0.1459,0.3466$ & $0.0393,0.0958$ & $0.0359,0.0892$ \\
\hline$S^{\mathrm{a}}$ & 1.023 & 1.254 & 1.040 & 1.098 \\
\hline $\begin{array}{l}\text { Parameters, } \\
\text { Restraints }\end{array}$ & 783,579 & 654,937 & 821,0 & 821,0 \\
\hline $\begin{array}{l}\text { Max.,min. } \\
\text { difference map, e } \\
\AA^{-3}\end{array}$ & $4.519,-3.285$ & $5.086,-5.950$ & $3.481,-2.303$ & $1.622,-1.887$ \\
\hline
\end{tabular}


Table S2. Crystallographic data for compounds 5-S3. ${ }^{a}$ Conventional $R=\Sigma|| F_{\mathrm{o}}|-| F \mathrm{c}|| / \Sigma\left|F_{\mathrm{o}}\right| ; R_{\mathrm{w}}=$ $\left[\Sigma \mathrm{w}\left(F_{\mathrm{o}}{ }^{2}-F_{\mathrm{c}}\right)^{2} / \Sigma \mid\left(F_{\mathrm{o}}^{2}\right)^{2}\right]^{1 / 2} ; S=\left[\Sigma \mathrm{w}\left(F_{\mathrm{o}}^{2}-F_{\mathrm{c}}^{2}\right)^{2} /(\text { no. data }- \text { no. parameters })\right]^{1 / 2}$ for all data.

\begin{tabular}{|c|c|c|c|c|}
\hline & 5 & S1 & S2 & S3 \\
\hline Formula & $\mathrm{C}_{53.50} \mathrm{H}_{82} \mathrm{IMoN}_{4} \mathrm{O}_{3} \mathrm{P}_{3} \mathrm{U}$ & $\mathrm{C}_{49} \mathrm{H}_{50} \mathrm{~K}_{2} \mathrm{~N}_{2} \mathrm{P}_{2}$ & $\mathrm{C}_{38} \mathrm{H}_{70} \mathrm{Li}_{2} \mathrm{~N}_{2} \mathrm{O}_{2} \mathrm{P}_{2}$ & $\mathrm{C}_{32} \mathrm{H}_{47} \mathrm{LiNO}_{2} \mathrm{P}$ \\
\hline Fw, $\mathrm{g} \mathrm{mol}^{-1}$ & 1383.01 & 807.05 & 662.78 & 515.61 \\
\hline Cryst size, $\mathrm{mm}$ & $0.116 \times 0.080 \times 0.028$ & $\begin{array}{lllll}0.243 & \mathrm{x} & 0.106 & \mathrm{x} \\
0.079 & & & \\
\end{array}$ & $\begin{array}{llll}0.509 & \mathrm{x} & 0.370 & \mathrm{x} \\
0.263 & & & \\
\end{array}$ & $\begin{array}{llll}0.620 & \mathrm{x} & 0.540 & \mathrm{x} \\
0.260 & & & \\
\end{array}$ \\
\hline Crystal system & triclinic & monoclinic & monoclinic & orthorhombic \\
\hline Space group & $P-1$ & $C 2 / \mathrm{c}$ & $P 2_{1} / \mathrm{c}$ & $P$ bca \\
\hline $\begin{array}{l}\text { Collection } \\
\text { Temperature (K) }\end{array}$ & $120(2)$ & $120(2)$ & $120(2)$ & $90(2)$ \\
\hline $\mathrm{a},(\AA)$ & $11.4147(5)$ & $19.4263(3)$ & $15.4396(4)$ & $16.5145(8)$ \\
\hline $\mathrm{b},(\AA)$ & $11.6195(5)$ & $10.28533(18)$ & $19.2492(6)$ & $17.9934(8)$ \\
\hline $\mathrm{c},(\AA)$ & $22.6570(9)$ & $21.7179(4)$ & $13.9739(5)$ & $20.8720(10)$ \\
\hline$\alpha,\left(^{\circ}\right)$ & $80.703(4)$ & 90 & 90 & 90 \\
\hline$\beta,\left(^{\circ}\right)$ & $79.216(4)$ & $97.7106(16)$ & $98.260(3)$ & 90 \\
\hline$\gamma,\left(^{\circ}\right)$ & $75.195(4)$ & 90 & 90 & 90 \\
\hline $\mathrm{V},\left(\AA^{3}\right)$ & $2833.5(2)$ & $4300.14(13)$ & $4110.0(2)$ & $6202.2(5)$ \\
\hline Z & 2 & 4 & 4 & 8 \\
\hline$\rho_{\text {calc }} \mathrm{g} \mathrm{cm}-^{3}$ & 1.621 & 1.247 & 1.071 & 1.104 \\
\hline$\mu, \mathrm{mm}^{-1}$ & 15.205 & 2.916 & 1.186 & 0.115 \\
\hline $\begin{array}{l}\text { No. of reflections } \\
\text { measured }\end{array}$ & 21886 & 18072 & 18934 & 51921 \\
\hline $\begin{array}{l}\text { No. of unique } \\
\text { reflections, Rint }\end{array}$ & $11277,0.0759$ & $4314,0.0318$ & $8192,0.0264$ & $7043,0.0208$ \\
\hline $\begin{array}{l}\text { No. of reflections } \\
\text { with } F^{2}>2 \mathrm{~s}\left(F^{2}\right)\end{array}$ & 8824 & 3865 & 6884 & 6203 \\
\hline $\begin{array}{l}\text { Transmission } \\
\text { coefficient range }\end{array}$ & $0.566-0.814$ & $0.58753-1.00000$ & $0.400-0.929$ & $0.678-0.746$ \\
\hline $\begin{array}{l}R, \quad R_{\mathrm{w}}{ }^{a} \quad\left(F^{2} \quad>\right. \\
\left.2 \mathrm{~s}\left(F^{2}\right)\right)\end{array}$ & $0.0714,0.1838$ & $0.0422,0.1135$ & $0.0547,0.1532$ & $0.0336,0.0884$ \\
\hline$R, R_{\mathrm{w}}{ }^{a}$ (all data) & $0.0895,0.1971$ & $0.0469,0.1190$ & $0.0635,0.1633$ & $0.0395,0.0939$ \\
\hline$S^{\mathrm{a}}$ & 1.059 & 1.030 & 1.039 & 1.037 \\
\hline $\begin{array}{l}\text { Parameters, } \\
\text { Restraints }\end{array}$ & 619,8 & 284,26 & 520,322 & 342,0 \\
\hline $\begin{array}{l}\text { Max.,min. } \\
\text { difference map, e } \\
\AA^{-3}\end{array}$ & $2.150,-4.736$ & $0.711,-0.245$ & $0.705,-0.465$ & $0.321,-0.254$ \\
\hline
\end{tabular}


Table S3. Crystallographic data for compounds S5-S8. ${ }^{a}$ Conventional $R=\Sigma|| F_{\mathrm{o}}|-| F_{\mathrm{c}}|| / \Sigma\left|F_{\mathrm{o}}\right| ; R_{\mathrm{w}}=$ $\left[\Sigma \mathrm{w}\left(F_{\mathrm{o}}{ }^{2}-F_{\mathrm{c}}\right)^{2} / \Sigma \mid\left(F_{\mathrm{o}}^{2}\right)^{2}\right]^{1 / 2} ; S=\left[\Sigma \mathrm{w}\left(F_{\mathrm{o}}^{2}-F_{\mathrm{c}}^{2}\right)^{2} /(\text { no. data }- \text { no. parameters })\right]^{1 / 2}$ for all data.

\begin{tabular}{|c|c|c|c|c|}
\hline & S5 & S6 & S7 & S8 \\
\hline Formula & $\mathrm{C}_{35} \mathrm{H}_{53} \mathrm{LiNO}_{2} \mathrm{P}$ & $\mathrm{C}_{29} \mathrm{H}_{53} \mathrm{LiNO}_{2} \mathrm{P}$ & $\mathrm{C}_{50} \mathrm{H}_{94} \mathrm{Li}_{2} \mathrm{~N}_{2} \mathrm{O}_{2} \mathrm{P}_{2}$ & $\mathrm{C}_{61} \mathrm{H}_{105} \mathrm{Cl}_{2} \mathrm{LiN}_{3} \mathrm{O}_{4} \mathrm{P}_{3} \mathrm{U}$ \\
\hline FW, $\mathrm{g} \mathrm{mol}^{-1}$ & 557.69 & 485.63 & 831.09 & 1353.25 \\
\hline Cryst size, $\mathrm{mm}$ & $0.68 \times 0.61 \times 0.27$ & $\begin{array}{l}0.2946 \times 0.2399 \times \\
0.2238\end{array}$ & $\begin{array}{l}0.3644 \times 0.1766 \times \\
0.137\end{array}$ & $0.280 \times 0.150 \times 0.050$ \\
\hline Crystal system & triclinic & orthorhombic & triclinic & Monoclinic \\
\hline Space group & $P-1$ & $P 2_{1} 2_{1} 2_{1}$ & $P-1$ & $C 2$ \\
\hline $\begin{array}{l}\text { Collection } \\
\text { Temperature (K) }\end{array}$ & $90(2)$ & $120(2)$ & $120(2)$ & $90(2)$ \\
\hline $\mathrm{a},(\AA)$ & $9.0356(4)$ & $10.1677(2)$ & $13.7992(5)$ & $23.396(13)$ \\
\hline $\mathrm{b},(\AA)$ & $9.7233(5)$ & $16.1908(4)$ & $14.4422(5)$ & $12.191(7)$ \\
\hline $\mathrm{c},(\AA)$ & $21.4962(10)$ & $18.1897(4)$ & $15.4016(6)$ & $24.124(13)$ \\
\hline$\alpha,\left(^{\circ}\right)$ & $87.510(2)$ & 90 & $78.977(3)$ & 90 \\
\hline$\beta,\left(^{\circ}\right)$ & $89.875(2)$ & 90 & $79.729(3)$ & $96.024(11)$ \\
\hline$\gamma,\left(^{\circ}\right)$ & $62.904(2)$ & 90 & $65.231(3)$ & 90 \\
\hline $\mathrm{V},\left(\AA^{3}\right)$ & $1679.37(14)$ & $2994.44(12)$ & $2718.94(18)$ & $6843(6)$ \\
\hline $\mathrm{Z}$ & 2 & 4 & 2 & 4 \\
\hline$\rho_{\text {calc }} \mathrm{g} \mathrm{cm}^{3}{ }^{3}$ & 1.103 & 1.077 & 1.015 & 1.314 \\
\hline$\mu, \mathrm{mm}^{-1}$ & 0.111 & 0.976 & 0.977 & 2.563 \\
\hline $\begin{array}{l}\text { No. of reflections } \\
\text { measured }\end{array}$ & 20483 & 12180 & 20713 & 16841 \\
\hline $\begin{array}{l}\text { No. of unique } \\
\text { reflections, Rint }\end{array}$ & $7514,0.0468$ & $5958,0.0227$ & $10731,0.0226$ & $10960,0.1148$ \\
\hline $\begin{array}{l}\text { No. of reflections } \\
\text { with } F^{2}>2 \mathrm{~s}\left(F^{2}\right)\end{array}$ & 5992 & 5745 & 9284 & 5540 \\
\hline $\begin{array}{l}\text { Transmission } \\
\text { coefficient range }\end{array}$ & $0.664-0.862$ & $0.946-0.956$ & $0.942-1.124$ & $0.2471-0.4305$ \\
\hline $\begin{array}{l}R, \quad R_{\mathrm{w}}{ }^{a} \quad\left(F^{2} \quad>\right. \\
\left.2 \mathrm{~s}\left(F^{2}\right)\right)\end{array}$ & $0.0525,0.1375$ & $0.0295,0.0746$ & $0.0443,0.1181$ & $0.1293,0.2804$ \\
\hline$R, R_{\mathrm{w}}{ }^{a}$ (all data) & $0.0654,0.1497$ & $0.0312,0.0763$ & $0.0519,0.1246$ & $0.2461,0.3542$ \\
\hline$S^{\mathrm{a}}$ & 1.035 & 1.044 & 1.028 & 1.011 \\
\hline $\begin{array}{l}\text { Parameters, } \\
\text { Restraints }\end{array}$ & 371,0 & 318,0 & 547,0 & 726,1358 \\
\hline $\begin{array}{l}\text { Max.,min. } \\
\text { difference map, e } \\
\AA^{-3}\end{array}$ & $0.694,-0.352$ & $0.176,-0.183$ & $0.673,-0.277$ & $5.562,-5.311$ \\
\hline
\end{tabular}


Table S4. Crystallographic data for compounds S9-S12. ${ }^{a}$ Conventional $R=\Sigma|| F_{\mathrm{o}}|-| F \mathrm{c}|| \Sigma\left|F_{\mathrm{O}}\right| ; R_{\mathrm{w}}$ $=\left[\Sigma \mathrm{w}\left(F_{\mathrm{o}}^{2}-F_{\mathrm{c}}^{2}\right)^{2} / \Sigma \mid\left(F_{\mathrm{o}}^{2}\right)^{2}\right]^{1 / 2} ; S=\left[\Sigma \mathrm{w}\left(F_{\mathrm{o}}^{2}-F_{\mathrm{c}}^{2}\right)^{2} /(\text { no. data }- \text { no. parameters })\right]^{1 / 2}$ for all data.

\begin{tabular}{|c|c|c|c|c|}
\hline & S9 & S10 & S11 & S12 \\
\hline Formula & $\mathrm{C}_{54} \mathrm{H}_{96} \mathrm{ClN}_{3} \mathrm{P}_{3} \mathrm{Th}$ & $\mathrm{C}_{45} \mathrm{H}_{75} \mathrm{IN}_{3} \mathrm{P}_{3} \mathrm{U}$ & $\mathrm{C}_{45} \mathrm{H}_{75} \mathrm{IN}_{3} \mathrm{P}_{3} \mathrm{Th}$ & $\mathrm{C}_{68} \mathrm{H}_{94} \mathrm{Cl}_{3} \mathrm{LiN}_{2} \mathrm{O}_{5} \mathrm{P}_{2} \mathrm{U}$ \\
\hline $\mathrm{Fw}, \mathrm{g} \mathrm{mol}^{-1}$ & 1147.73 & 1115.92 & 1109.93 & 1432.71 \\
\hline Cryst size, mm & $\begin{array}{lllll}0.306 & \mathrm{x} & 0.238 & \mathrm{x} \\
0.184 & & & & \\
\end{array}$ & $0.12 \times 0.11 \times 0.02$ & $\begin{array}{l}0.2192 \times 0.1576 \times \\
0.0986\end{array}$ & $\begin{array}{lllll}0.390 & \mathrm{x} & 0.320 & \mathrm{x} \\
0.260 & & & \\
\end{array}$ \\
\hline Crystal system & triclinic & triclinic & triclinic & triclinic \\
\hline Space group & $P-1$ & $P-1$ & $P-1$ & $P 1$ \\
\hline $\begin{array}{l}\text { Collection } \\
\text { Temperature (K) }\end{array}$ & $120(2)$ & $90(2)$ & $120(2)$ & $150(2)$ \\
\hline $\mathrm{a},(\AA)$ & $10.9070(2)$ & $11.033(4)$ & $11.1478(4)$ & $12.3941(4)$ \\
\hline $\mathrm{b},(\AA)$ & $16.4743(4)$ & $11.169(4)$ & $11.2199(4)$ & $12.8821(4)$ \\
\hline $\mathrm{c},(\AA)$ & $16.9387(5)$ & $21.187(7)$ & $21.3286(9)$ & 13.0494(4) \\
\hline$\alpha,\left(^{\circ}\right)$ & $92.820(2)$ & $76.611(5)$ & $76.574(3)$ & $109.4340(10)$ \\
\hline$\beta,\left(^{\circ}\right)$ & $106.089(2)$ & $88.002(5)$ & $87.975(3)$ & $110.8610(10)$ \\
\hline$\gamma,\left(^{\circ}\right)$ & $100.1909(19)$ & $68.623(5)$ & $68.688(3)$ & $100.1430(10)$ \\
\hline $\mathrm{V},\left(\AA^{3}\right)$ & $2862.71(13)$ & $2361.5(13)$ & $2413.82(17)$ & $1730.36(10)$ \\
\hline $\mathrm{Z}$ & 2 & 2 & 2 & 1 \\
\hline$\rho_{\text {calc }} \mathrm{g} \mathrm{cm}^{3}{ }^{3}$ & 1.332 & 1.569 & 1.527 & 1.375 \\
\hline$\mu, \mathrm{mm}^{-1}$ & 9.873 & 4.225 & 16.153 & 2.554 \\
\hline $\begin{array}{l}\text { No. of reflections } \\
\text { measured }\end{array}$ & 21163 & 16761 & 17978 & 21282 \\
\hline $\begin{array}{l}\text { No. of unique } \\
\text { reflections, Rint }\end{array}$ & $11296,0.0290$ & $8242,0.0733$ & $9537,0.0297$ & $13113,0.0202$ \\
\hline $\begin{array}{l}\text { No. of reflections } \\
\text { with } F^{2}>2 \mathrm{~s}\left(F^{2}\right)\end{array}$ & 10842 & 5658 & 9190 & 13080 \\
\hline $\begin{array}{l}\text { Transmission } \\
\text { coefficient range }\end{array}$ & $0.432-0.559$ & $0.3976-0.5553$ & $0.376-0.575$ & $0.372-0.43$ \\
\hline $\begin{array}{llll}R, & R_{\mathrm{W}}{ }^{a} & \left(F^{2}\right. & > \\
\left.2 \mathrm{~s}\left(F^{2}\right)\right) & & \\
\end{array}$ & $0.0303,0.0793$ & $0.0618,0.1083$ & $0.0297,0.0719$ & $0.0203,0.0461$ \\
\hline$R, R_{\mathrm{w}}{ }^{a}$ (all data) & $0.0317,0.0804$ & $0.1042,0.1193$ & $0.0307,0.0728$ & $0.0206,0.0463$ \\
\hline$S^{\mathrm{a}}$ & 1.104 & 1.011 & 1.064 & 0.729 \\
\hline $\begin{array}{l}\text { Parameters, } \\
\text { Restraints }\end{array}$ & 577,101 & 489,682 & 499,0 & 842,396 \\
\hline $\begin{array}{l}\text { Max.,min. } \\
\text { difference map, e } \\
\AA^{-3}\end{array}$ & $1.729,-1.011$ & $1.998,-3.651$ & $3.583,-2.237$ & $1.070,-0.318$ \\
\hline
\end{tabular}


Table S5. Crystallographic data for compounds S13 and S14. ${ }^{a}$ Conventional $R=\Sigma|| F_{\mathrm{o}}|-| F \mathrm{c}||$

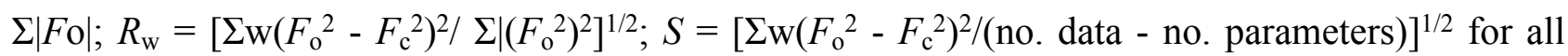
data.

\begin{tabular}{|c|c|c|}
\hline & S13 & S14 \\
\hline Formula & $\mathrm{C}_{54} \mathrm{H}_{93} \mathrm{ClN}_{3} \mathrm{P}_{3} \mathrm{U}$ & $\mathrm{C}_{70} \mathrm{H}_{98} \mathrm{Cl}_{3} \mathrm{LiN}_{2} \mathrm{O}_{4} \mathrm{P}_{2} \mathrm{U}$ \\
\hline FW, $\mathrm{g} \mathrm{mol}^{-1}$ & 1150.70 & 1444.76 \\
\hline Cryst size, $\mathrm{mm}$ & $0.11 \times 0.07 \times 0.04$ & $\begin{array}{llll}0.210 & \mathrm{x} & 0.160 & \mathrm{X} \\
0.150 & & & \\
\end{array}$ \\
\hline Crystal system & monoclinic & orthorhombic \\
\hline Space group & $P 2_{1} / \mathrm{n}$ & $P 2_{1} 2_{1} 2_{1}$ \\
\hline $\begin{array}{l}\text { Collection } \\
\text { Temperature (K) }\end{array}$ & $90(2)$ & $90(2)$ \\
\hline $\mathrm{a},(\AA)$ & $13.231(5)$ & $23.4842(13)$ \\
\hline $\mathrm{b},(\AA)$ & $19.466(7)$ & $12.3263(7)$ \\
\hline $\mathrm{c},(\AA)$ & $21.880(8)$ & $24.2038(15)$ \\
\hline$\alpha,\left(^{\circ}\right)$ & 90 & 90 \\
\hline$\beta,\left(^{\circ}\right)$ & $95.484(6)$ & 90 \\
\hline$\gamma,\left(^{\circ}\right)$ & 90 & 90 \\
\hline $\mathrm{V},\left(\AA^{3}\right)$ & $5609(3)$ & $7006.4(7)$ \\
\hline $\mathrm{Z}$ & 4 & 4 \\
\hline$\rho_{\text {calc }} \mathrm{g} \mathrm{cm}^{3}{ }^{3}$ & 1.363 & 1.370 \\
\hline$\mu, \mathrm{mm}^{-1}$ & 3.062 & 2.523 \\
\hline $\begin{array}{l}\text { No. of reflections } \\
\text { measuredd }\end{array}$ & 26729 & 86073 \\
\hline $\begin{array}{l}\text { No. of unique } \\
\text { reflections, Rint }\end{array}$ & $9878,0.0967$ & $15896,0.1396$ \\
\hline $\begin{array}{l}\text { No. of reflections } \\
\text { with } F^{2}>2 \mathrm{~s}\left(F^{2}\right)\end{array}$ & 6209 & 12614 \\
\hline $\begin{array}{l}\text { Transmission } \\
\text { coefficient range }\end{array}$ & $0.336-0.431$ & $0.3066-0.4305$ \\
\hline$R, R_{\mathrm{w}}{ }^{a}\left(F^{2}>2 \mathrm{~s}\left(F^{2}\right)\right)$ & $0.0624,0.1348$ & $0.0608,0.1343$ \\
\hline$R, R_{\mathrm{w}}{ }^{a}$ (all data) & $0.1182,0.1540$ & $0.0842,0.1464$ \\
\hline$S^{\mathrm{a}}$ & 1.002 & 1.037 \\
\hline $\begin{array}{l}\text { Parameters, } \\
\text { Restraints }\end{array}$ & 583,852 & 780,123 \\
\hline $\begin{array}{l}\text { Max.,min. } \\
\text { difference map, e } \AA^{-}\end{array}$ & $1.614,-4.035$ & $3.740,-2.586$ \\
\hline
\end{tabular}




\section{Supplementary References}

1. Sheldrick, G. M. A short history of SHELX. Acta Cryst. Sect. A 2008, A64 (1), 112-122.

2. Sheldrick, G. M. SHELXT- Integrated space-group and crystal-structure determination. Acta Cryst. Sect. A 2015, A71. 3-8.

3. Dolomanov O. V.; Bourhis L. J.; Gildea R. J.; Howard J. A. K.; Puschmann, H. OLEX2: a complete structure solution, refinement and analysis program. J. Appl. Cryst. 2009, 42, 339341.

4. CrysAlisPRO version 39.46, Oxford Diffraction/Agilent Technologies UK Ltd, Yarnton, England.

5. Bruker 2012. APEX, SMART, SAINT-Plus. Bruker AXS Inc., Madison, Wisconsin, USA.

6. Sheldrick, G. M. Crystal structure refinement with SHELXL. Acta Cryst. Sect. C 2015, C71. 3-8.

7. Farugia, L.J. WinGX and ORTEP for windows: an update. J. Appl. Cryst. 2012, 45, 849-854.

8. Persistence of Vision ${ }^{\mathrm{TM}}$ Raytracer, Persistence of Vision Pty. Ltd., Williamstown, Victoria, Australia.

9. Poetschke, N.; Nieger, M.; Khan, M. A.; Niecke, E.; Ashby, M. T. Synthesis and Crystal Structures of Lithium Salts of New Iminophosphide/Phosphinoamide Anions. Inorg. Chem. 1997, 36, 4087-4093.

10. Ayres, A. J.; Zegke, M.; Ostrowski, J. P. A.; Tuna, F.; McInnes, E. J. L.; Wooles, A. J.; Liddle, S. T. Actinide-Transition Metal Bonding in Heterobimetallic Uranium- and ThoriumMolybdenum Paddlewheel Complexes. Chem. Commun. 2018, 54, 13515-13518.

11. Napoline, J. W.; Kraft, S. J.; Matson, E. M.; Fanwick, P. E.; Bart, S. C.; Thomas, C. M. Tris(Phosphinoamide)-Supported Uranium-Cobalt Heterobimetallic Complexes Featuring $\mathrm{Co} \rightarrow \mathrm{U}$ Dative Interactions. Inorg. Chem. 2013, 52, 12170-12177. 